\title{
SCHREIER SETS IN RAMSEY THEORY
}

\author{
V. FARMAKI AND S. NEGREPONTIS
}

\begin{abstract}
We show that Ramsey theory, a domain presently conceived to guarantee the existence of large homogeneous sets for partitions on $k$-tuples of words (for every natural number $k$ ) over a finite alphabet, can be extended to one for partitions on Schreier-type sets of words (of every countable ordinal). Indeed, we establish an extension of the partition theorem of Carlson about words and of the (more general) partition theorem of Furstenberg-Katznelson about combinatorial subspaces of the set of words (generated from $k$-tuples of words for any fixed natural number $k$ ) into a partition theorem about combinatorial subspaces (generated from Schreier-type sets of words of order any fixed countable ordinal). Furthermore, as a result we obtain a strengthening of Carlson's infinitary Nash-Williams type (and Ellentuck type) partition theorem about infinite sequences of variable words into a theorem, in which an infinite sequence of variable words and a binary partition of all the finite sequences of words, one of whose components is, in addition, a tree, are assumed, concluding that all the Schreier-type finite reductions of an infinite reduction of the given sequence have a behavior determined by the CantorBendixson ordinal index of the tree-component of the partition, falling in the tree-component above that index and in its complement below it.
\end{abstract}

\section{INTRODUCTION}

Our aim is to extend Ramsey theory so that it applies not only to partitions of $k$-tuples of words but more generally to partitions of Schreier-type sets of words of a fixed countable ordinal number. For a finite non-empty alphabet $\Sigma$ we denote by $W^{k}(\Sigma)$ (respectively, $W^{k}(\Sigma ; v)$ ) the family of sequences of $k$ many words (respectively, variable words) over $\Sigma$, and by $W^{\omega}(\Sigma ; v)$ the family of infinite sequences of variable words over $\Sigma$. By a reduction (respectively, variable reduction) of $\vec{w}=\left(w_{n}\right)_{n \in \mathbb{N}} \in W^{\omega}(\Sigma ; v)$ we mean any infinite sequence of words (respectively, variable words), denoted by $\vec{u} \prec \vec{w}$, obtained from $\vec{w}$ by replacing each occurence of the variable in each $w_{n}$ by one element of the set $\Sigma \cup\{v\}$, dividing the resulting sequence into infinitely many finite blocks of consecutive words, and concatenating the members of each block; the first element (respectively, the first $k$ elements) of a reduction of $\vec{w}$ is called a reduced word (respectively, a finite reductions with $k$ words) of $\vec{w}$. (These terms will be defined more formally below.) For a natural number $r$, an $r$-coloring (or an $r$-partition) of a set $S$ is a map $\chi: S \rightarrow\{1, \ldots, r\}$, and $\chi(s)$ is the color of $s$ for $s \in S$. A set $T \subseteq S$ is monochromatic (under $\chi$ ) if $\chi$ is constant on $T$.

Received by the editors October 23, 2005.

2000 Mathematics Subject Classification. Primary 05D10.

Key words and phrases. Ramsey theory, Schreier sets, words. 
The fundamental classical partition theorems of Ramsey theory, namely (a) Carlson's partition theorem (Lemma 5.9 in [C], Corollary 4.6 in $[\mathrm{BBH}]$ in strengthened form), (b) the Furstenberg-Katznelson partition theorem (Theorems 2.7 and 3.1 in $[\mathrm{FK}]$ ), and (c) Carlson's Nash-Williams type infinitary partition theorem (Theorem 2 in $[\mathrm{C}])$, can now be stated as follows:

Theorem 1.1 (Carlson's theorem, $[\mathrm{C}],[\mathrm{BBH}])$. Let $\chi_{1}: W^{1}(\Sigma) \rightarrow\left\{1, \ldots, r_{1}\right\}$ and $\chi_{2}: W^{1}(\Sigma ; v) \rightarrow\left\{1, \ldots, r_{2}\right\}$ be finite colorings of the sets $W^{1}(\Sigma)$ and $W^{1}(\Sigma ; v)$, respectively and $\vec{w} \in W^{\omega}(\Sigma ; v)$ be an infinite sequence of variable words over $\Sigma$. Then there exists a variable reduction $\vec{u} \prec \vec{w}$ of $\vec{w}$ such that all the reduced words of $\vec{u}$ are monochromatic under $\chi_{1}$ and all the reduced variable words of $\vec{u}$ are monochromatic under $\chi_{2}$.

Theorem 1.2 (Furstenberg-Katznelson's theorem, $[\mathrm{FK}])$. Let $k$ be any natural number, $\chi_{1}: W^{k}(\Sigma) \rightarrow\left\{1, \ldots, r_{1}\right\}$ and $\chi_{2}: W^{k}(\Sigma ; v) \rightarrow\left\{1, \ldots, r_{2}\right\}$ be finite colorings of the sets $W^{k}(\Sigma)$ and $W^{k}(\Sigma ; v)$, respectively and $\vec{w} \in W^{\omega}(\Sigma ; v)$ be an infinite sequence of variable words over $\Sigma$. Then there exists a variable reduction $\vec{u} \prec \vec{w}$ of $\vec{w}$ such that all the finite reductions with $k$ words of $\vec{u}$ are monochromatic under $\chi_{1}$ and all the finite variable reductions with $k$ variable words of $\vec{u}$ are monochromatic under $\chi_{2}$.

In addition Furstenberg and Katznelson in $[\mathrm{FK}]$ introduced the notion of a $k$ dimensional combinatorial subspace of $W(\Sigma)$ for $k$ any natural number and proved (in Theorem 3.1) a partition theorem about these combinatorial subspaces.

Theorem 1.3 (Carlson's infinitary partition theorem, $[\mathrm{C}])$. Let $\mathcal{U} \subseteq W^{\omega}(\Sigma ; v)$ be a pointwise closed family (as defined immediately after Theorem 4.6 below) of infinite sequences of variable words over $\Sigma$ and $\vec{w} \in W^{\omega}(\Sigma ; v)$ be an infinite sequence of variable words over $\Sigma$. Then there exists a variable reduction $\vec{u} \prec \vec{w}$ of $\vec{w}$ over $\Sigma$ such that either all the variable reductions of $\vec{u}$ are contained in $\mathcal{U}$ or all variable reductions of $\vec{u}$ are contained in the complement of $\mathcal{U}$.

As stated, the aim of the present paper is to show that stronger versions of these partition theorems hold for the family of Schreier-type sets of words of every countable ordinal, and not just for the family of $k$-tuples of words, with $k$ restricted to a natural number. The hierarchy $\left(\mathcal{A}_{\xi}\right)_{\xi<\omega_{1}}$ of the families of Schreier sets of natural numbers, defined on the countable ordinals, provides a classification of the class of all finite subsets of the natural numbers measuring their complexity. The recursive definition of the Schreier sets $\left(\mathcal{A}_{\xi}\right)_{\xi<\omega_{1}}$ is as follows (where by $[\mathbb{N}]_{>0}^{<\omega}$ we denote, as explained in detail in Section 1 below, the set of all non-empty finite subsets of the set $\mathbb{N}=\{1,2, \ldots\}$ of natural numbers):

We denote by $\mathbb{N}=\{1,2, \ldots\}$ the set of natural numbers, $[\mathbb{N}]_{>0}^{<\omega}$ the set of all non-empty, finite subsets of $\mathbb{N},[\mathbb{N}]^{<\omega}=[\mathbb{N}]_{>0}^{<\omega} \cup\{\emptyset\}$ and $[\mathbb{N}]^{\omega}$ the set of all infinite subsets of $\mathbb{N}$.

Definition 1.4 (The Schreier system, [F1, Def. 7], [F2, Def. 1.5], [F3, Def. 1.3]). For every non-zero, countable, limit ordinal $\lambda$ choose and fix a strictly increasing sequence $\left(\lambda_{n}\right)_{n \in \mathbb{N}}$ of successor ordinals smaller than $\lambda$ with $\sup _{n} \lambda_{n}=\lambda$. The system $\left(\mathcal{A}_{\xi}\right)_{\xi<\omega_{1}}$ is defined recursively as follows:

(1) $\mathcal{A}_{0}=\{\emptyset\}$ and $\mathcal{A}_{1}=\{\{n\}: n \in \mathbb{N}\}$;

(2) $\mathcal{A}_{\zeta+1}=\left\{s \in[\mathbb{N}]_{>0}^{<\omega}: s=\{n\} \cup s_{1}\right.$, where $n \in \mathbb{N},\{n\}<s_{1}$ and $\left.s_{1} \in \mathcal{A}_{\zeta}\right\}$; 
(3i) $\mathcal{A}_{\omega^{\beta+1}}=\left\{s \in[\mathbb{N}]_{>0}^{<\omega}: s=\bigcup_{i=1}^{n} s_{i}\right.$, where $n=\min s_{1}, s_{1}<\cdots<s_{n}$ and $\left.s_{1}, \ldots, s_{n} \in \mathcal{A}_{\omega^{\beta}}\right\}$

(3ii) for a non-zero, countable limit ordinal $\lambda$,

$$
\mathcal{A}_{\omega^{\lambda}}=\left\{s \in[\mathbb{N}]_{>0}^{<\omega}: s \in \mathcal{A}_{\omega^{\lambda_{n}}} \operatorname{with} n=\min s\right\}
$$

and

(3iii) for a limit ordinal $\xi$ such that $\omega^{\alpha}<\xi<\omega^{\alpha+1}$ for some $0<\alpha<\omega_{1}$, if $\xi=\omega^{\alpha} p+\sum_{i=1}^{m} \omega^{a_{i}} p_{i}$, where $m \in \mathbb{N}$ with $m \geq 0, p, p_{1}, \ldots, p_{m}$ are natural numbers with $p, p_{1}, \ldots, p_{m} \geq 1$ (so that either $p>1$, or $p=1$ and $m \geq 1$ ) and $a, a_{1}, \ldots, a_{m}$ are ordinals with $a>a_{1}>\cdots a_{m}>0$, $\mathcal{A}_{\xi}=\left\{s \in[\mathbb{N}]_{>0}^{<\omega}: s=s_{0} \cup\left(\bigcup_{i=1}^{m} s_{i}\right)\right.$ with $s_{m}<\cdots<s_{1}<s_{0}$, $s_{0}=s_{1}^{0} \cup \cdots \cup s_{p}^{0}$ with $s_{1}^{0}<\cdots<s_{p}^{0} \in \mathcal{A}_{\omega^{a}}$, and $s_{i}=s_{1}^{i} \cup \cdots \cup s_{p_{i}}^{i}$ with $\left.s_{1}^{i}<\cdots<s_{p_{i}}^{i} \in \mathcal{A}_{\omega^{a_{i}}} \forall 1 \leq i \leq m\right\}$.

Note that in case (3iii) above the Cantor normal form of ordinals is employed (cf. [KM], [L]).

It is important to note that $\mathcal{A}_{k}=[\mathbb{N}]^{k}$, the family of all $k$-element subsets of the natural numbers, for every $k \in \mathbb{N}$, i.e. for every finite ordinal $k<\omega$; thus the families of Schreier sets $\mathcal{A}_{\xi}$ for $\xi<\omega_{1}$ constitute the natural transfinite analogues of the $k$-element subsets of $\mathbb{N}$. On the other hand, for every ordinal $\xi \geq \omega$ the family $\mathcal{A}_{\xi}$ differs radically from the families $\mathcal{A}_{k}$ for all $k<\omega$, in that it contains finite sets of arbitrarily large cardinality.

The families $\mathcal{A}_{\omega}=\left\{s \in[\mathbb{N}]_{>0}^{<\omega}: s \in[\mathbb{N}]^{k}\right.$, where $\left.k=\min s\right\}$, and $\mathcal{A}_{\omega^{n+1}}=\{s \in$ $[\mathbb{N}]_{>0}^{<\omega}: s=\bigcup_{i=1}^{k} s_{i}$, where $\left.k=\min s_{1}, s_{1}<\cdots<s_{k} \in \mathcal{A}_{\omega^{n}}\right\}$ are determined uniquely by case (3i) of Definition 1.4. In general however the Schreier families are not defined in a unique way, but depend ultimately on the choice of the converging sequence $\left(\lambda_{n}\right)$ to the limit ordinals $\lambda$. This choice bears directly on case (3ii) and indirectly on all other cases of Definition 1.4. The first real choice is for the limit ordinal $\lambda=\omega$ and, assuming that the sequence $\left(\lambda_{n}\right)$ converging to $\omega$ is the sequence with $\lambda_{n}=n, \mathcal{A}_{\omega^{\omega}}=\left\{s \in[\mathbb{N}]_{>0}^{<\omega}: s \in \mathcal{A}_{\omega^{k}}\right.$ with $\left.k=\min s\right\}$. By choosing "natural" converging sequences $\lambda_{n}=\omega+n$ to limit ordinal $\lambda=\omega 2, \ldots, \lambda_{n}=\omega(n-1)+n$ to limit ordinal $\lambda=\omega^{\omega}$, the families $\mathcal{A}_{\omega^{\omega 2}}, \ldots, \mathcal{A}_{\omega^{\omega}}$, are defined respectively. More "natural" converging sequences are considered in Section 2 of [KS] (up to the ordinal $\left.\epsilon_{0}\right)$ and in Chapter VII of $[\mathrm{KM}]$. However, it appears impossible to make canonical, natural choices for all limit ordinals, and it thus seems that the definition of the recursive system of thin Schreier families $\left(\mathcal{A}_{\xi}\right)_{\xi<\omega_{1}}$ depends essentially on the arbitrary choices of (many) converging sequences.

It must be emphasised however that our results (in Sections 2 to 5 below) do not depend on the particular choice of the converging sequences. The basic feature that differentiates the $\mathcal{A}_{\xi}$ 's from each other is complexity: in fact, irrespectively of the particular choices of the converging sequences employed for the definition of the family $\mathcal{A}_{\xi}$, the Cantor-Bendixson index of the compact subset (in the Cantor set $\{0,1\}^{\mathbb{N}}$, under the canonical identification of a set with its characteristic function) corresponding to $\mathcal{A}_{\xi}$ is precisely $\xi+1$ (as proved in [F3, Proposition 2.9]). Thus the complexity of the family $\mathcal{A}_{\xi}$, as measured by its Cantor-Bendixson index, is independent of the particular choices of the converging sequences (cf. Proposition 4.12). This invariance is in fact needed for the results in Sections 3 to 5 below.

Although the recursive Schreier system $\left(\mathcal{A}_{\xi}\right)_{\xi<\omega_{1}}$ is a purely combinatorial entity, it nevertheless arose gradually in connection with the theory of Banach spaces. 
Originally the family $\mathcal{A}_{\xi}$ was defined by Schreier ([S]) (for $\xi=\omega$ ), next by AlspachOdell [AO] (for $\xi=\omega^{\kappa}, \kappa$ a natural number) and Alspach-Argyros [AA] (for $\xi=$ $\omega^{\alpha}, \alpha$ a countable ordinal), and finally by Farmaki [F1], [F2], [F3] and TomczakJaegermann $[\mathrm{TJ}]$ (for $\xi$ any countable ordinal). (The reader is referred to the introduction of [F3] for more details.)

Schreier sets were first used for the following transfinite extension of the classical Ramsey partition theorem $([\mathrm{R}])$, a result about the existence of monochromatic sets for finite colorations of the family of all $k$-tuples, with $k$ a natural number:

Theorem 1.5 (Ramsey partition theorem on Schreier sets, ([F2])). Let $\xi$ be a nonzero countable ordinal number. For any finite coloration $\chi$ of the family $\mathcal{A}_{\xi}$ and $M$ an infinite subset of $\mathbb{N}$ there exists an infinite subset $L$ of $M$ such that $\mathcal{A}_{\xi} \cap[L]^{<\omega}$ is monochromatic.

Using the family $\mathcal{A}_{\xi}$ we define (in Definition 3.1) the families $W^{\xi}(\Sigma), W^{\xi}(\Sigma ; v)$ of Schreier-type sets of words, variable words respectively over $\Sigma$, of a fixed countable ordinal number $\xi$. Carlson's theorem (Theorem 1.1) and the more general Furstenberg-Katznelson's theorem (Theorem 1.2) will be extended from $k$-tuples to Schreier-type sets of every countable ordinal; this is the content of the main Theorem in Section 2 (see Theorem 3.3). With the notation and definitions given in Section 1, it reads as follows:

Theorem A. Let $\xi$ be a countable ordinal, $\chi_{1}: W^{\xi}(\Sigma) \rightarrow\left\{1, \ldots, r_{1}\right\}$ and $\chi_{2}$ : $W^{\xi}(\Sigma ; v) \rightarrow\left\{1, \ldots, r_{2}\right\}$ be finite colorings of the sets $W^{\xi}(\Sigma)$ and $W^{\xi}(\Sigma ; v)$, respectively and $\vec{w} \in W^{\omega}(\Sigma ; v)$ be an infinite sequence of variable words over $\Sigma$. Then there exists a variable reduction $\vec{u} \prec \vec{w}$ of $\vec{w}$ such that all the finite reductions of $\vec{u}$ in the set $W^{\xi}(\Sigma)$ are monochromatic under $\chi_{1}$ and all the finite variable reductions of $\vec{u}$ in the set $W^{\xi}(\Sigma ; v)$ are monochromatic under $\chi_{2}$.

The proof of this result is closer to the method employed by us in proving Schreier-type extensions of Hindman's and Milliken-Taylor's theorems in [FN], which in turn is inspired by the method invented by Baumgartner to prove Hindman's theorem in [B]; in particular, we do not use topological dynamics (as employed in $[\mathrm{FK}]$ ) or idempotent ultrafilters (as employed in $[\mathrm{C}],[\mathrm{BBH}]$ ). Some consequences of the Main Theorem are described in Section 2. Beside the Carlson and the Furstenberg-Katznelson theorems, Schreier-type extensions of the Hale-Jewett's theorem $([\mathrm{HJ}])$ and consequently of the van der Waerden's theorem ([vdW]) are obtained.

Theorem A is next used, in conjuction with the tools developed in Section 3, one of which is a suitable Cantor-Bendixson index, to strengthen Carlson's infinitary theorem (Theorem 1.3) to various forms of Nash-Williams type partition theorems for words and variable words, involving Schreier families. A somewhat weaker version of our main results (Theorems 5.2, 5.4) is contained in the following statement, which also strengthens Theorem 1.3 (see Remark 5.6).

Theorem B. Let $\mathcal{G} \subseteq W^{<\omega}(\Sigma)$ and $\mathcal{F} \subseteq W^{<\omega}(\Sigma ; v)$ be trees and $\vec{w} \in W^{\omega}(\Sigma ; v)$ be an infinite sequence of variable words over $\Sigma$. Then either there exists a variable reduction $\vec{u} \prec \vec{w}$ of $\vec{w}$ such that all the finite reductions of $\vec{u}$ over $\Sigma$ are contained in $\mathcal{G}$ or there exists a countable ordinal $\xi_{1}=\zeta_{\vec{w}}^{\mathcal{G}}$ such that for all $\xi>\xi_{1}$ there exists a variable reduction $\vec{u} \prec \vec{w}$ of $\vec{w}$ such that all the finite reductions of $\vec{u}$ in the set $W^{\xi}(\Sigma)$ are contained in the complement of $\mathcal{G}$. 
Furthermore, either there exists a variable reduction $\vec{u} \prec \vec{w}$ of $\vec{w}$ such that all the finite variable reductions of $\vec{u}$ over $\Sigma$ are contained in $\mathcal{F}$ or there exists a countable ordinal $\xi_{2}=\zeta_{\vec{w}}^{\mathcal{F}}$ such that for all $\xi>\xi_{2}$ there exists a variable reduction $\vec{u} \prec \vec{w}$ of $\vec{w}$ such that all the finite variable reductions of $\vec{u}$ in the set $W^{\xi}(\Sigma ; v)$ are contained in the complement of $\mathcal{F}$.

Theorem B is strengthened, involving the Ellentuck topology $\mathfrak{T}_{E}$ in Theorem 6.2. A simple consequence of Theorem 6.2 is the characterization of completely Ramsey partitions of $W^{\omega}(\Sigma)$ and $W^{\omega}(\Sigma ; v)$ in terms of the Baire property in the topology $\mathfrak{T}_{E}$, a result proved with different methods by Carlson in [C].

Let us remark at this point that the attractive alternative approach to Ramsey theory, via located words rather than 'classical' words, given by Bergelson-BlassHindman in $[\mathrm{BBH}]$, also admits a Schreier-type extension, analogous to the one given in the present paper. The details will appear elsewhere.

The extended Ramsey theory developed in the present paper is a more powerful tool than the 'classical' Ramsey theory in that Schreier sets of all countable-ordinal orders capture a considerable part of analysis, which is beyond the reach of the arithmetically oriented 'classical' Ramsey theory. This is attested by the fact that the Schreier families have found essential applications in Banach space theory on such questions as, for example, unconditionality, $l^{1}$ and $c_{0}$ embeddability, and distortion (see e.g. [F1], [O], [AGR], [F4]).

It is also noteworthy that the hereditary family $\left(\mathcal{A}_{\omega}\right)_{*}=\left\{t \in[\mathbb{N}]^{<\omega}: t \subseteq s\right.$ for some $\left.s \in \mathcal{A}_{\omega}\right\} \cup\{\emptyset\}$ generated by $\mathcal{A}_{\omega}$ figures prominently (under the name of the family of "not large" sets) in questions of mathematical logic related to concrete realisations of Godel's incompleteness theorem, specifically in the (Ramsey type) Paris-Harrington statements, statements true and provable in set-theory but unprovable in Peano arithmetic (cf. [PH], [KS] and [GRS], pp. 169-180). (Here the choice of sequences $\left(\lambda_{n}\right)_{n \in \mathbb{N}}$ increasing to a limit ordinal $\lambda$ in Definition 1.4 is crucial.) The higher order hereditary Schreier families $\left(\mathcal{A}_{\xi}\right)_{*}$, and specifically a suitable finitary form of Theorem 1.5 involving sets in these families, might well be useful in forming and proving statements true but unprovable in certain systems endowed with induction stronger than that in Peano arithmetic.

The fact that Ramsey theory has found important applications in various branches of mathematics (including mathematical logic, Banach space theory, Ramsey ergodic theory) makes it reasonable to expect that the Schreier-type extension of Ramsey theory presented in this paper will have interesting applications.

\section{Terminology AND NOTATiON}

We develop in this section the necessary terminology and notation. We denote by $\mathbb{N}=\{1,2, \ldots\}$ the set of natural numbers, $[\mathbb{N}]_{>0}^{<\omega}$ the set of all non-empty, finite subsets of $\mathbb{N},[\mathbb{N}]^{<\omega}=[\mathbb{N}]_{>0}^{<\omega} \cup\{\emptyset\}$ and $[\mathbb{N}]^{\omega}$ the set of all infinite subsets of $\mathbb{N}$.

Let $\Sigma$ be a finite, non-empty alphabet, and $v \notin \Sigma$ an entity which we call a variable. A word over $\Sigma$ is a finite sequence of elements of $\Sigma$. The set of all the words over $\Sigma$ is denoted by $W(\Sigma)$; thus

$$
W(\Sigma)=\left\{w=\alpha_{1} \ldots \alpha_{k}: k \in \mathbb{N}, \alpha_{1}, \ldots, \alpha_{k} \in \Sigma\right\} .
$$

$W(\Sigma)$ is turned into a semigroup by the operation of concatenation: the concatenation of two words $w_{1}=\alpha_{1} \ldots \alpha_{k}, w_{2}=\beta_{1} \ldots \beta_{l}$ over $\Sigma$ is defined to be the 
word

$$
w_{1} * w_{2}=\alpha_{1} \ldots \alpha_{k} \beta_{1} \ldots \beta_{l} .
$$

For two words $w_{1}=\alpha_{1} \ldots \alpha_{k}, w_{2}=\beta_{1} \ldots \beta_{l}$ over $\Sigma$ we write

$$
w_{1} \propto w_{2} \text { iff } k<l \text { and } \alpha_{i}=\beta_{i} \text { for } i=1, \ldots, k,
$$

and in case $w_{1} \propto w_{2}$ we set $w_{2}-w_{1}=\beta_{k+1} \ldots \beta_{l} \in W(\Sigma)$.

A variable word over $\Sigma$ is a word over $\Sigma \cup\{v\}$ in which $v$ actually appears. So, the set $W(\Sigma ; v)$ of variable words over $\Sigma$ is defined as

$$
W(\Sigma ; v)=W(\Sigma \cup\{v\}) \backslash W(\Sigma) .
$$

We note that the concatenation of two variable words is also a variable word. If $w$ is a variable word over $\Sigma$ and $\alpha \in \Sigma \cup\{v\}$, then we write $w(\alpha)$ for the result of replacing every occurence of the variable $v$ in $w$ by $\alpha$. Thus $w(\alpha) \in W(\Sigma)$ for $\alpha \in \Sigma$ and $w(v)=w$. For two variable words $w_{1}=\alpha_{1} \ldots \alpha_{k}, w_{2}=\beta_{1} \ldots \beta_{l}$ over $\Sigma$ we write

$w_{1} \propto w_{2}$ iff $k<l, \alpha_{i}=\beta_{i}$ for $i=1, \ldots, k$ and $w_{2}-w_{1}=\beta_{k+1} \ldots \beta_{l} \in W(\Sigma ; v)$.

We denote by $W^{<\omega}(\Sigma)$ the family of all finite sequences of words over the alphabet $\Sigma$, by $W^{\omega}(\Sigma)$ the family of all infinite sequences of words over $\Sigma$ and by $W^{<\omega}(\Sigma ; v), W^{\omega}(\Sigma ; v)$ the families of all finite, infinite sequences of variable words over $\Sigma$ respectively. Hence,

$$
\begin{aligned}
& W^{<\omega}(\Sigma)=\left\{\mathbf{w}=\left(w_{1}, \ldots, w_{l}\right): l \in \mathbb{N}, w_{1}, \ldots, w_{l} \in W(\Sigma)\right\} \cup\{\emptyset\}, \\
& W^{<\omega}(\Sigma ; v)=\left\{\mathbf{w}=\left(w_{1}, \ldots, w_{l}\right): l \in \mathbb{N}, w_{1}, \ldots, w_{l} \in W(\Sigma ; v)\right\} \cup\{\emptyset\}, \\
& W^{\omega}(\Sigma)=\left\{\vec{w}=\left(w_{n}\right)_{n \in \mathbb{N}}: w_{n} \in W(\Sigma) \text { for every } n \in \mathbb{N}\right\} \\
& W^{\omega}(\Sigma ; v)=\left\{\vec{w}=\left(w_{n}\right)_{n \in \mathbb{N}}: w_{n} \in W(\Sigma ; v) \text { for every } n \in \mathbb{N}\right\} .
\end{aligned}
$$

The complexity of a finite sequence $\mathbf{w}=\left(w_{1}, \ldots, w_{l}\right) \in W^{<\omega}(\Sigma \cup\{v\}) \backslash\{\emptyset\}$ of words, with $w_{i}=\alpha_{k_{i}} \alpha_{k_{i}+1} \ldots \alpha_{k_{i+1}-1}$ for $i=1, \ldots, l$, is described by the complexity of the corresponding finite sequence of natural numbers $1=k_{1}<\cdots<k_{l}<k_{l+1} \in \mathbb{N}$, a complexity that will be described by the Schreier hierarchy; we thus define the correspondence

$$
\begin{gathered}
d: W^{<\omega}(\Sigma \cup\{v\}) \backslash\{\emptyset\} \rightarrow[\mathbb{N}]^{<\omega} \text { such that } \mathbf{w}=\left(w_{1}, \ldots, w_{l}\right) \rightarrow d(\mathbf{w}) \\
\text { with } d(\mathbf{w})=\emptyset \text { if } l=1, \text { and } d(\mathbf{w})=\left\{k_{2}<k_{3}<\cdots<k_{l}\right\} \text { if } l>1 .
\end{gathered}
$$

Analogously, for every infinite sequence $\vec{w}=\left(w_{n}\right)_{n \in \mathbb{N}} \in W^{\omega}(\Sigma \cup\{v\})$ of words, with $w_{n}=\alpha_{k_{n}} \alpha_{k_{n}+1} \ldots \alpha_{k_{n+1}-1}$ for all $n \in \mathbb{N}$, the corresponding complexity is described by the complexity of the infinite sequence $1=k_{1}<k_{2}<k_{3}<\cdots \in \mathbb{N}$ of natural numbers; we thus define the correspondence

$$
d: W^{\omega}(\Sigma \cup\{v\}) \rightarrow[\mathbb{N}]^{\omega} \text { with } d\left(\left(w_{n}\right)_{n \in \mathbb{N}}\right)=\left(k_{2}<k_{3}<\cdots<k_{n}<\cdots\right) .
$$

A finite sequence $\mathbf{w}=\left(w_{1}, \ldots, w_{l}\right) \in W^{<\omega}(\Sigma \cup\{v\})$ is an initial segment of the finite sequence $\mathbf{u}=\left(u_{1}, \ldots, u_{k}\right) \in W^{<\omega}(\Sigma \cup\{v\})$ iff $l<k$ and $u_{i}=w_{i}$ for $i=1, \ldots, l$ and $\mathbf{w}$ is an initial segment of the infinite sequence $\vec{u}=\left(u_{n}\right)_{n \in \mathbb{N}} \in W^{\omega}(\Sigma \cup\{v\})$ if $u_{i}=w_{i}$ for all $i=1, \ldots, l$. In these cases, extending to sequences the previous notation for words, we write $\mathbf{w} \propto \mathbf{u}$ or $\mathbf{w} \propto \vec{u}$, and we set

$$
\mathbf{u} \backslash \mathbf{w}=\left(u_{l+1}, \ldots, u_{k}\right) \text { and } \vec{u} \backslash \mathbf{w}=\left(u_{n}\right)_{n>l} \text { respectively. }
$$


Definition 2.1. (1) (Reduction of an infinite sequence of words by a word) For an infinite sequence $\vec{w}=\left(w_{n}\right)_{n \in \mathbb{N}} \in W^{\omega}(\Sigma ; v)$ of variable words and for a (variable or non-variable) word $t=\alpha_{1} \ldots \alpha_{k} \in \mathcal{W}(\Sigma \cup\{v\})$ (over the alphabet $\Sigma$ ), we set

$$
\vec{w}[t]=w_{1}\left(\alpha_{1}\right) * \ldots * w_{k}\left(\alpha_{k}\right) \in \mathcal{W}(\Sigma \cup\{v\}) .
$$

The family $R W(\vec{w})$ of all the reduced words and the family $V R W(\vec{w})$ of all the variable reduced words of $\vec{w}$ over $\Sigma$ are defined as follows:

$$
R W(\vec{w})=\{\vec{w}[t]: t \in W(\Sigma)\} \text { and } V R W(\vec{w})=\{\vec{w}[t]: t \in W(\Sigma ; v)\} .
$$

For $u_{1}=\vec{w}\left[t_{1}\right], u_{2}=\vec{w}\left[t_{2}\right] \in R W(\vec{w})$ (resp. $\left.u_{1}, u_{2} \in V R W(\vec{w})\right)$ we write

$$
u_{1} \propto u_{2} \text { iff } t_{1} \propto t_{2} .
$$

(2) (Reduction of an infinite sequence of words by a finite sequence of words) For an infinite sequence $\vec{w}=\left(w_{n}\right)_{n \in \mathbb{N}} \in W^{\omega}(\Sigma ; v)$ of variable words and for a finite sequence of (variable or non-variable) words $\mathbf{t}=\left(t_{1}, \ldots, t_{l}\right) \in W^{<\omega}(\Sigma \cup\{v\}) \backslash\{\emptyset\}$, with $t_{i}=\alpha_{k_{i}} \alpha_{k_{i}+1} \ldots \alpha_{k_{i+1}-1}$ for all $i=1, \ldots, l$, we set

$$
\vec{w}[\mathbf{t}]=\left(u_{1}, \ldots, u_{l}\right) \in W^{<\omega}(\Sigma \cup\{v\}),
$$

where

$$
u_{i}=w_{k_{i}}\left(\alpha_{k_{i}}\right) * w_{k_{i}+1}\left(\alpha_{k_{i}+1}\right) * \ldots * w_{k_{i+1}-1}\left(\alpha_{k_{i+1}-1}\right) \text { for all } i=1, \ldots, l .
$$

Also we set $\vec{w}[\emptyset]=\emptyset$. The finite sequences of words $\vec{w}[\mathbf{t}]$ for $\mathbf{t} \in W^{<\omega}(\Sigma)$ are called finite reductions of $\vec{w}$ over $\Sigma$, and the finite sequences of variable words $\vec{w}[\mathbf{t}]$ for $\mathbf{t} \in W^{<\omega}(\Sigma ; v)$ are called finite variable reductions of $\vec{w}$ over $\Sigma$. The set of all the finite reductions and the set of all the finite variable reductions of $\vec{w}$ over $\Sigma$ are denoted as follows:

$$
R W^{<\omega}(\vec{w})=\left\{\vec{w}[\mathbf{t}]: \mathbf{t} \in W^{<\omega}(\Sigma)\right\} \text { and } V R W^{<\omega}(\vec{w})=\left\{\vec{w}[\mathbf{t}]: \mathbf{t} \in W^{<\omega}(\Sigma ; v)\right\} .
$$

We set

$$
d_{\vec{w}}: R W^{<\omega}(\vec{w}) \cup V R W^{<\omega}(\vec{w}) \backslash\{\emptyset\} \rightarrow[\mathbb{N}]^{<\omega} \text { with } d_{\vec{w}}(\vec{w}[\mathbf{t}])=d(\mathbf{t}) .
$$

Observe that $R W^{<\omega}(\vec{e})=W^{<\omega}(\Sigma), V R W^{<\omega}(\vec{e})=W^{<\omega}(\Sigma ; v)$ and $d_{\vec{e}}=d$ if $\vec{e}=$ $\left(e_{n}\right)_{n \in \mathbb{N}}$ with $e_{n}=v$ for every $n \in \mathbb{N}$. Note also that it is not always true that $d_{\vec{w}}(\mathbf{u})=d(\mathbf{u})$ for every $\mathbf{u} \in R W^{<\omega}(\vec{w})$.

(3) (Reduction of an infinite sequence of words by an infinite sequence of words) For an infinite sequence $\vec{w}=\left(w_{n}\right)_{n \in \mathbb{N}} \in W^{\omega}(\Sigma ; v)$ of variable words and for an infinite sequence $\vec{t}=\left(t_{n}\right)_{n \in \mathbb{N}} \in W^{\omega}(\Sigma \cup\{v\})$ of (variable or non-variable) words, with $t_{n}=\alpha_{k_{n}} \alpha_{k_{n}+1} \ldots \alpha_{k_{n+1}-1}$ for all $n \in \mathbb{N}$, we set

$$
\vec{w}[\vec{t}]=\left(u_{n}\right)_{n \in \mathbb{N}} \in W^{\omega}(\Sigma \cup\{v\}),
$$

where

$$
u_{n}=w_{k_{n}}\left(\alpha_{k_{n}}\right) * w_{k_{n}+1}\left(\alpha_{k_{n}+1}\right) * \ldots * w_{k_{n+1}-1}\left(\alpha_{k_{n+1}-1}\right) \text { for all } n \in \mathbb{N} .
$$

An infinite sequence of words $\vec{w}[\vec{t}]$ for $\vec{t} \in W^{\omega}(\Sigma)$ is called a reduction of $\vec{w}$ over $\Sigma$ and for $\vec{t} \in W^{\omega}(\Sigma ; v)$ a variable reduction of $\vec{w}$ over $\Sigma$, respectively. The sets of all the reductions and all the variable reductions of $\vec{w}$ over $\Sigma$ respectively are denoted as follows:

$$
R W^{\omega}(\vec{w})=\left\{\vec{w}[\vec{t}]: \vec{t} \in W^{\omega}(\Sigma)\right\} \text { and } V R W^{\omega}(\vec{w})=\left\{\vec{w}[\vec{t}]: \vec{t} \in W^{\omega}(\Sigma ; v)\right\} .
$$

For $\vec{u}, \vec{w} \in W^{\omega}(\Sigma ; v)$, we write

$$
\vec{u} \prec \vec{w} \text { if and only if } \vec{u} \in V R W^{\omega}(\vec{w}) .
$$


Notice that $\vec{u} \prec \vec{w}$ if and only if $V R W(\vec{u}) \subseteq V R W(\vec{w})$. Hence, $\vec{w} \prec \vec{e}$ for every $\vec{w} \in W^{<\omega}(\Sigma ; v)$ in case $\vec{e}=\left(e_{n}\right)_{n \in \mathbb{N}}$ with $e_{n}=v$ for every $n \in \mathbb{N}$. We define

$$
d_{\vec{w}}: R W^{\omega}(\vec{w}) \cup V R W^{\omega}(\vec{w}) \rightarrow[\mathbb{N}]^{\omega} \text { with } d_{\vec{w}}(\vec{w}[\vec{t}])=d(\vec{t}) .
$$

$\left(1^{*}\right)$ (Reduction of a finite sequence of words by a word) For a finite sequence $\mathbf{w}=\left(w_{1}, \ldots, w_{n}\right) \in \mathcal{W}^{<\omega}(\Sigma ; v)$ of variable words over the alphabet $\Sigma$, we define the sets

$$
\begin{aligned}
& R W\left(\left(w_{1}, \ldots, w_{n}\right)\right)=\left\{w_{1}\left(\alpha_{1}\right) * \ldots * w_{n}\left(\alpha_{n}\right): \alpha_{1} \ldots \alpha_{n} \in W(\Sigma)\right\} \text { and } \\
& V R W\left(\left(w_{1}, \ldots, w_{n}\right)\right)=\left\{w_{1}\left(\alpha_{1}\right) * \ldots * w_{n}\left(\alpha_{n}\right): \alpha_{1} \ldots \alpha_{n} \in W(\Sigma ; v)\right\}
\end{aligned}
$$

of all the reduced words and variable reduced words, respectively, of $\left(w_{1}, \ldots, w_{n}\right)$ over $\Sigma$.

Notice that $R W\left(\left(w_{1}, \ldots, w_{n}\right)\right), V R W\left(\left(w_{1}, \ldots, w_{n}\right)\right)$ are finite sets and that for a sequence $\vec{w}=\left(w_{n}\right)_{n \in \mathbb{N}} \in W^{\omega}(\Sigma ; v)$ we have that $R W(\vec{w})=\bigcup\left\{R W\left(w_{1}, \ldots, w_{n}\right)\right.$ : $n \in \mathbb{N}\}$ and $V R W(\vec{w})=\bigcup\left\{V R W\left(w_{1}, \ldots, w_{n}\right): n \in \mathbb{N}\right\}$.

$\left(2^{*}\right)$ (Reduction of a finite sequence of words by a finite sequence of words) For a finite sequence $\mathbf{w}=\left(w_{1}, \ldots, w_{n}\right) \in W^{<\omega}(\Sigma ; v)$ of variable words over the alphabet $\Sigma$, we define analogously the families $R W^{<\omega}(\mathbf{w})$ and $V R W^{<\omega}(\mathbf{w})$ of all finite reductions and variable finite reductions, respectively, of $\mathbf{w}$ over $\Sigma$. So, $\left(u_{1}, \ldots, u_{l}\right) \in R W^{<\omega}(\mathbf{w})$ if there exists $\mathbf{t}=\left(t_{1}, \ldots, t_{l}\right) \in W^{<\omega}(\Sigma)$, where $t_{i}=$ $\alpha_{k_{i}} \alpha_{k_{i}+1} \ldots \alpha_{k_{i+1}-1}$ for all $i=1, \ldots, l$ and $k_{l+1}=n+1$, such that

$$
u_{i}=w_{k_{i}}\left(\alpha_{k_{i}}\right) * w_{k_{i}+1}\left(\alpha_{k_{i}+1}\right) * \ldots * w_{k_{i+1}-1}\left(\alpha_{k_{i+1}-1}\right) \text { for } i=1, \ldots, l .
$$

We set $d_{\mathbf{w}}(\mathbf{u})=\left\{k_{2}, \ldots, k_{l}\right\}$.

In the sequel we will also employ the following notation. For the families $\mathcal{G} \subseteq$ $W^{<\omega}(\Sigma), \mathcal{F} \subseteq W^{<\omega}(\Sigma ; v)$ and the words $s \in W(\Sigma), t \in W(\Sigma ; v)$ we set

$$
\begin{gathered}
\mathcal{G}(s)=\left\{\mathbf{w} \in W^{<\omega}(\Sigma): \text { either } \mathbf{w}=\left(w_{1}, \ldots, w_{l}\right) \neq \emptyset, s \propto w_{1}\right. \text { and } \\
\left.\left(s, w_{1}-s, w_{2}, \ldots, w_{l}\right) \in \mathcal{G} \text { or } \mathbf{w}=\emptyset \text { and }(s) \in \mathcal{G}\right\}, \text { and } \\
\mathcal{F}(t)=\left\{\mathbf{w} \in W^{<\omega}(\Sigma ; v): \text { either } \mathbf{w}=\left(w_{1}, \ldots, w_{l}\right), t \propto w_{1}\right. \text { and } \\
\left.\left(t, w_{1}-t, w_{2}, \ldots, w_{l}\right) \in \mathcal{F}, \text { or } \mathbf{w}=\emptyset \text { and }(t) \in \mathcal{F}\right\} .
\end{gathered}
$$

Also,

$$
\begin{gathered}
\mathcal{G}-s=\left\{\mathbf{w} \in \mathcal{G}: \text { either } \mathbf{w}=\left(w_{1}, \ldots, w_{l}\right) \text { and } s \propto w_{1}, \text { or } \mathbf{w}=\emptyset\right\} \text { and } \\
\mathcal{F}-t=\left\{\mathbf{w} \in \mathcal{F}: \text { either } \mathbf{w}=\left(w_{1}, \ldots, w_{l}\right) \text { and } t \propto w_{1}, \text { or } \mathbf{w}=\emptyset\right\}
\end{gathered}
$$

For the sequence $\vec{w}=\left(w_{n}\right)_{n \in \mathbb{N}} \in W^{\omega}(\Sigma ; v)$ and the words $t \in V R W(\vec{w})$, $s \in R W(\vec{w}))$ with $t \in V R W\left(\left(w_{1}, \ldots, w_{k}\right)\right)$ and $s \in R W\left(\left(w_{1}, \ldots, w_{k}\right)\right)$ for some $k \in \mathbb{N}$, we set

$$
\begin{aligned}
& \vec{w}-t=\left(w_{0}, w_{k+2}, w_{k+3}, \ldots\right) \in V R W^{\omega}(\vec{w}), \text { where } w_{0}=t * w_{k+1} \text {, and } \\
& \qquad \vec{w}-s=\left(w_{0}, w_{k+2}, w_{k+3}, \ldots\right) \in V R W^{\omega}(\vec{w}) \text {, where } w_{0}=s * w_{k+1} .
\end{aligned}
$$

Also, we set $\vec{w} \backslash t=\vec{w} \backslash s=\left(w_{k+1}, w_{k+2}, \ldots\right) \in \mathcal{W}^{\omega}(\Sigma ; v)$. 


\section{The main partition theorem on Schreier families}

The main theorem of this section is Theorem 3.3, given in equivalent form in Theorem 3.6. This is a partition theorem for the Schreier finite sequences of words and the Schreier finite sequences of variable words over a finite non-empty alphabet $\Sigma$ of every countable order, and constitutes an extension to every countable order $\xi$ (a) of Carlson's theorem, Theorem 1.1, corresponding to ordinal level $\xi=0$ and (b) of Theorem 1.2, proved by Furstenberg and Katznelson, corresponding to finite ordinals $\xi<\omega$.

In order to state Theorem 3.3 we need the following definitions:

Definition 3.1 (The Schreier systems $\left(W^{\xi}(\Sigma)\right)_{0 \leq \xi<\omega_{1}}$ and $\left.\left(W^{\xi}(\Sigma ; v)\right)_{0 \leq \xi<\omega_{1}}\right)$. Let $\left(\mathcal{A}_{\xi}\right)_{\xi<\omega_{1}}$ be a Schreier system of families of finite subsets of $\mathbb{N}$ and $\Sigma$ be a finite alphabet. We will define the families $W^{\xi}(\Sigma)$ and $W^{\xi}(\Sigma ; v)$ of the Schreier finite sequences of words and of variable words over $\Sigma$ respectively, for every countable ordinal $\xi$ recursively as follows:

$$
W^{0}(\Sigma)=\left\{\mathbf{w}=\left(w_{1}\right): w_{1} \in W(\Sigma)\right\} \text { and } W^{0}(\Sigma ; v)=\left\{\mathbf{w}=\left(w_{1}\right): w_{1} \in W(\Sigma ; v)\right\}
$$

and for every countable ordinal $\xi \geq 1$

$$
\begin{aligned}
& W^{\xi}(\Sigma)=\left\{\mathbf{w}=\left(w_{1}, \ldots, w_{l}\right) \in W^{<\omega}(\Sigma): d\left(\left(w_{1}, \ldots, w_{l}\right)\right) \in \mathcal{A}_{\xi}\right\} ; \text { and }, \\
& W^{\xi}(\Sigma ; v)=\left\{\mathbf{w}=\left(w_{1}, \ldots, w_{l}\right) \in W^{<\omega}(\Sigma ; v): d\left(\left(w_{1}, \ldots, w_{l}\right)\right) \in \mathcal{A}_{\xi}\right\} .
\end{aligned}
$$

For an infinite sequence $\vec{w}=\left(w_{n}\right)_{n \in \mathbb{N}} \in \mathcal{W}^{\omega}(\Sigma ; v)$ of variable words over $\Sigma$, we define the families of Schreier finite reductions and of variable reductions of $\vec{w}$ over $\Sigma$ as follows:

$$
R W^{0}(\vec{w})=\left\{\mathbf{u}=\left(u_{1}\right): u_{1} \in R W(\vec{w})\right\} \text { and } V R W^{0}(\vec{w})=\left\{\mathbf{u}=\left(u_{1}\right): u_{1} \in V R W(\vec{w})\right\},
$$

and for every countable ordinal $\xi \geq 1$

$$
\begin{gathered}
R W^{\xi}(\vec{w})=\left\{\mathbf{u}=\left(u_{1}, \ldots, u_{l}\right) \in R W^{<\omega}(\vec{w}): d_{\vec{w}}\left(\left(u_{1}, \ldots, u_{l}\right)\right) \in \mathcal{A}_{\xi}\right\} ; \text { and }, \\
V R W^{\xi}(\vec{w})=\left\{\mathbf{u}=\left(u_{1}, \ldots, u_{l}\right) \in V R W^{<\omega}(\vec{w}): d_{\vec{w}}\left(\left(u_{1}, \ldots, u_{l}\right)\right) \in \mathcal{A}_{\xi}\right\} .
\end{gathered}
$$

Hence, a finite sequence $\mathbf{w} \in W^{<\omega}(\Sigma)$ of words over $\Sigma$ belongs to the family $W^{\xi}(\Sigma)$ for some $1 \leq \xi<\omega_{1}$ iff $\mathbf{w}=\left(w_{1}, \ldots, w_{l}\right)$ with $l>1$, and there exist $1=k_{1}<\cdots<k_{l}<k_{l+1} \in \mathbb{N}$ with $\left\{k_{i}: 2 \leq i \leq l\right\} \in \mathcal{A}_{\xi}$ and $\alpha_{1}, \ldots, \alpha_{k_{l+1}-1} \in \Sigma$ such that $w_{i}=\alpha_{k_{i}} \alpha_{k_{i}+1} \ldots \alpha_{k_{i+1}-1}$ for all $i=1, \ldots, l$.

Observe that $\mathbf{w}=(w) \in W^{0}(\Sigma)$ for every $w \in W(\Sigma)$, while $\mathbf{w}=(w) \notin W^{\xi}(\Sigma)$ for every $\xi>0$. Also observe that $W^{\xi}(\Sigma)=R W^{\xi}(\vec{e})$ and $W^{\xi}(\Sigma ; v)=V R W^{\xi}(\vec{e})$ for every countable ordinal $\xi$, in case $\vec{e}=\left(e_{n}\right)_{n \in \mathbb{N}}$ with $e_{n}=v$ for every $n \in \mathbb{N}$, and that it is not true that $W^{\xi}(\Sigma)=R W^{\xi}(\vec{w})$ for every $\vec{w} \in W^{\omega}(\Sigma ; v)$.

The following proposition expresses the recursiveness of the Schreier systems $\left(W^{\xi}(\Sigma)\right)_{0 \leq \xi<\omega_{1}}$ and $\left(W^{\xi}(\Sigma ; v)\right)_{0 \leq \xi<\omega_{1}}$.

Proposition 3.2. For every countable ordinal $\xi>0$ there exists a concrete sequence $\left(\xi_{n}\right)_{n>1}$ of countable ordinals with $\xi_{n}<\xi$ for every $n \in \mathbb{N}, 1<n$ such that

$W^{\xi}(\Sigma)(s)=W^{\xi_{n}}(\Sigma) \cap\left(W^{<\omega}(\Sigma)-s\right)$ and $W^{\xi}(\Sigma ; v)(t)=W^{\xi_{n}}(\Sigma ; v) \cap\left(W^{<\omega}(\Sigma ; v)-t\right)$ for every $n \in \mathbb{N}, 1<n$ and $s=\alpha_{1} \ldots \alpha_{n-1} \in W(\Sigma), t=\beta_{1} \ldots \beta_{n-1} \in W(\Sigma ; v)$.

Moreover, $\xi_{n}=\zeta$ for every $n \in \mathbb{N}$ in case $\xi=\zeta+1$, and $\left(\xi_{n}\right)$ is a strictly increasing sequence with $\sup _{n} \xi_{n}=\xi$ in case $\xi$ is a limit ordinal. 
Proof. According to Proposition 1.7 in [F3], for every countable ordinal $\xi>0$ there exists a concrete sequence $\left(\xi_{n}\right)$ of countable ordinals with $\xi_{n}<\xi$ such that

$$
\mathcal{A}_{\xi}(n)=\mathcal{A}_{\xi_{n}} \cap[\{n+1, n+2, \ldots\}]^{<\omega} \text { for every } n \in \mathbb{N},
$$

where, $\mathcal{A}_{\xi}(n)=\left\{s \in[\mathbb{N}]^{<\omega}:\{n\}<s,\{n\} \cup s \in \mathcal{A}_{\xi}\right\}$ for every $n \in \mathbb{N}$. Moreover, $\xi_{n}=\zeta$ for every $n \in \mathbb{N}$ if $\xi=\zeta+1$, and $\left(\xi_{n}\right)$ is a strictly increasing sequence with $\sup _{n} \xi_{n}=\xi$ if $\xi$ is a limit ordinal.

Let $n>1$ and $s=\alpha_{1} \ldots \alpha_{n-1} \in W(\Sigma), t=\beta_{1} \ldots \beta_{n-1} \in W(\Sigma ; v)$. We will prove that $W^{\xi}(\Sigma)(s)=W^{\xi_{n}}(\Sigma) \cap\left(W^{<\omega}(\Sigma)-s\right)$ for every countable ordinal $\xi>0$. Similarly it can be proved that $W^{\xi}(\Sigma ; v)(t)=W^{\xi_{n}}(\Sigma ; v) \cap\left(W^{<\omega}(\Sigma ; v)-t\right)$ for every $0<\xi<\omega_{1}$.

For $\xi=1$, of course $W^{1}(\Sigma)(s)=W^{0}(\Sigma) \cap\left(W^{<\omega}(\Sigma)-s\right)$. Let $1<\xi<\omega_{1}$. Then $\emptyset \notin W^{\xi}(\Sigma)(s)$, since if $\emptyset \in W^{\xi}(\Sigma)(s)$, then $(s) \in W^{\xi}(\Sigma)$ for $\xi>0$ and of course $\emptyset \notin W^{\xi_{n}}(\Sigma)$ for every $0 \leq \xi_{n}<\omega_{1}$. Let $\mathbf{w}=\left(w_{1}, \ldots, w_{l}\right) \in W^{<\omega}(\Sigma) \backslash\{\emptyset\}$. Then there exist $1=k_{1}<\cdots<k_{l}<k_{l+1} \in \mathbb{N}$ and $\alpha_{1}, \ldots, \alpha_{k_{l+1}-1} \in \Sigma$ such that $w_{i}=\alpha_{k_{i}} \alpha_{k_{i}+1} \ldots \alpha_{k_{i+1}-1}$ for all $i=1, \ldots, l$.

If $\mathbf{w} \in W^{\xi}(\Sigma)(s)$, then $s \propto w_{1}$ and $\left(s, w_{1}-s, \ldots, w_{l}\right) \in W^{\xi}(\Sigma)$. In case $l>1$, we have that $n<k_{2}$ and that $\left\{n, k_{2}, \ldots, k_{l}\right\} \in \mathcal{A}_{\xi}$. So, $\left\{k_{2}, \ldots, k_{l}\right\} \in \mathcal{A}_{\xi_{n}}$ and consequently $\left(w_{1}, w_{2}, \ldots, w_{l}\right) \in W^{\xi_{n}}(\Sigma) \cap\left(W^{<\omega}(\Sigma)-s\right)$. In case $\mathbf{w}=\left(w_{1}\right) \in$ $W^{\xi}(\Sigma)(s)$, we have that $s \propto w_{1}$ and $\left(s, w_{1}-s\right) \in W^{\xi}(\Sigma)$. Thus $\{n\} \in \mathcal{A}_{\xi}$ and consequently $\emptyset \in \mathcal{A}_{\xi_{n}}$. This implies $\xi_{n}=0$ and indeed $\mathbf{w} \in W^{\xi_{n}}(\Sigma) \cap\left(\mathcal{W}^{<\omega}(\Sigma)-s\right)$.

If $\mathbf{w}=\left(w_{1}, \ldots, w_{l}\right) \in W^{\xi_{n}}(\Sigma) \cap\left(W^{<\omega}(\Sigma)-s\right)$ and $l>1$, then $\left\{k_{2}, \ldots, k_{l}\right\} \in$ $\mathcal{A}_{\xi_{n}} \cap[\{n+1, n+2, \ldots\}]^{<\omega} \subseteq \mathcal{A}_{\xi}(n)$. Since, $\left\{n, k_{2}, \ldots, k_{l}\right\} \in \mathcal{A}_{\xi}$, we have that $\left(s, w_{1}-s, \ldots, w_{k}\right) \in W^{\xi}(\Sigma)$ and consequently $\mathbf{w} \in W^{\xi}(\Sigma)(s)$. In case $\mathbf{w}=\left(w_{1}\right) \in$ $W^{\xi_{n}}(\Sigma) \cap\left(W^{<\omega}(\Sigma)-s\right)$, we have that $\xi_{n}=0$ and consequently $\{n\} \in \mathcal{A}_{\xi}$. Hence $\left(s, w_{1}-s\right) \in W^{\xi}(\Sigma)$ and $\mathbf{w} \in W^{\xi}(\Sigma)(s)$.

Now we can state and prove the main theorem of this section.

Theorem 3.3 (A partition theorem on Schreier sets of words). Let $\xi$ be a countable ordinal and $\Sigma$ a finite non-empty alphabet. For every $\mathcal{G} \subseteq W^{<\omega}(\Sigma), \mathcal{F} \subseteq$ $W^{<\omega}(\Sigma ; v)$ and every infinite sequence $\vec{w} \in W^{\omega}(\Sigma ; v)$ of variable words over $\Sigma$ there exists a variable reduction $\vec{u} \prec \vec{w}$ of $\vec{w}$ over $\Sigma$ such that :

either $W^{\xi}(\Sigma) \cap R W^{<\omega}(\vec{u}) \subseteq \mathcal{G}$, or $W^{\xi}(\Sigma) \cap R W^{<\omega}(\vec{u}) \subseteq W^{<\omega}(\Sigma) \backslash \mathcal{G}$, and either $W^{\xi}(\Sigma ; v) \cap V R W^{<\omega}(\vec{u}) \subseteq \mathcal{F}$, or $\mathcal{W}^{\xi}(\Sigma ; v) \cap V R W^{<\omega}(\vec{u}) \subseteq \mathcal{W}^{<\omega}(\Sigma ; v) \backslash \mathcal{F}$.

For the proof of this partition theorem we will make use of a diagonal argument, contained in the following lemmas.

Lemma 3.4. Let $\vec{w}=\left(w_{n}\right)_{n \in \mathbb{N}} \in W^{\omega}(\Sigma ; v)$ be an infinite sequence of variable words over the alphabet $\Sigma$ and let $\Pi_{1}=\{(s, \vec{u}): s \in R W(\vec{w})$ and $\vec{u} \prec \vec{w} \backslash s\}$. If a subset $\mathcal{R}$ of $\Pi_{1}$ satisfies:

(i) for every $(s, \vec{u}) \in \Pi_{1}$ there exists $\left(s, \vec{u}_{1}\right) \in \mathcal{R}$ with $\vec{u}_{1} \prec \vec{u}$; and

(ii) for every $\left(s, \vec{u}_{1}\right) \in \mathcal{R}$ and $\vec{u}_{2} \prec \vec{u}_{1}$ we have $\left(s, \vec{u}_{2}\right) \in \mathcal{R}$,

then there exists $\vec{u} \prec \vec{w}$ such that $(s, \vec{s}) \in \mathcal{R}$ for all $s \in R W(\vec{u})$ and $\vec{s} \prec \vec{u} \backslash s$.

Proof. Let $u_{0}=w_{1}$ and $\vec{u}_{0}=\vec{w}$. According to conditions (i) and (ii), there exists $\vec{u}_{1}=\left(u_{n}^{1}\right)_{n \in \mathbb{N}} \in W^{\omega}(\Sigma ; v)$ such that $\vec{u}_{1} \prec \vec{w} \backslash u_{0}$ and $\left(u_{0}(\alpha), \vec{u}_{1}\right) \in \mathcal{R}$ for every $\alpha \in \Sigma$. Let $u_{1}=u_{1}^{1}$. Then $\left(u_{0}, u_{1}\right) \in V R W^{<\omega}(\vec{w})$. We assume now that there have been constructed $\vec{u}_{1}, \ldots, \vec{u}_{n} \in W^{\omega}(\Sigma ; v)$ and $u_{0}, u_{1}, \ldots, u_{n} \in W(\Sigma ; v)$ such that 
$\left(u_{0}, u_{1}, \ldots, u_{n}\right) \in V R W^{<\omega}(\vec{w}), u_{i} \in V R W\left(\vec{u}_{i}\right), \vec{u}_{i} \prec \vec{u}_{i-1} \backslash u_{i-1}$ for each $1 \leq i \leq n$ and $\left(s, \vec{u}_{i}\right) \in \mathcal{R}$ for all $s \in R W\left(\left(u_{0}, \ldots, u_{i-1}\right)\right)$ and $1 \leq i \leq n$.

We will construct $\vec{u}_{n+1}$ and $u_{n+1}$. Let $R W\left(\left(u_{0}, \ldots, u_{n}\right)\right)=\left\{s_{1}, \ldots, s_{k}\right\}$ for some $k \in \mathbb{N}$. Then $\left(s_{i}, \vec{u}\right) \in \Pi_{1}$ for every $\vec{u} \prec \vec{u}_{n} \backslash u_{n}$ and $i=1, \ldots, k$. According to condition (i), there exist $\vec{u}_{n+1}^{1}, \ldots, \vec{u}_{n+1}^{k} \in W^{\omega}(\Sigma ; v)$ such that $\vec{u}_{n+1}^{k} \prec \cdots \prec$ $\vec{u}_{n+1}^{1} \prec \vec{u}_{n} \backslash u_{n}$ and $\left(s_{i}, \vec{u}_{n+1}^{i}\right) \in \mathcal{R}$ for every $1 \leq i \leq k$. Set $\vec{u}_{n+1}=\vec{u}_{n+1}^{k}$. If $\vec{u}_{n+1}=\left(u_{i}^{n+1}\right)_{i \in \mathbb{N}}$, then set $u_{n+1}=u_{1}^{n+1}$. Of course $u_{n+1} \in V R W\left(\vec{u}_{n+1}\right)$, $\vec{u}_{n+1} \prec \vec{u}_{n} \backslash u_{n},\left(u_{0}, u_{1}, \ldots, u_{n+1}\right) \in V R W^{<\omega}(\vec{w})$ and, according to condition (ii), $\left(s_{i}, \vec{u}_{n+1}\right) \in \mathcal{R}$ for all $1 \leq i \leq k$.

Set $\vec{u}=\left(u_{0}, u_{1}, u_{2}, \ldots\right) \in W^{\omega}(\Sigma ; v)$. Since $\left(u_{0}, u_{1}, \ldots, u_{n}\right) \in V R W^{<\omega}(\vec{w})$ for every $n \in \mathbb{N}$, we have that $\vec{u} \prec \vec{w}$. Let $s \in R W(\vec{u})$ and $\vec{s} \prec \vec{u} \backslash s$. Then there exists $n \in \mathbb{N}$ such that $s \in R W\left(\left(u_{0}, u_{1}, \ldots, u_{n}\right)\right)$. Thus $\left(s, \vec{u}_{n+1}\right) \in \mathcal{R}$. Since $\vec{u} \backslash s \prec \vec{u}_{n+1}$, according to (ii), $(s, \vec{u} \backslash s) \in \mathcal{R}$. So $(s, \vec{s}) \in \mathcal{R}$, since $\vec{s} \prec \vec{u} \backslash s$.

Lemma 3.5. Let $\vec{w}=\left(w_{n}\right)_{n \in \mathbb{N}} \in W^{\omega}(\Sigma ; v)$ be an infinite sequence of variable words over the alphabet $\Sigma$ and $\Pi_{2}=\{(t, \vec{u}): t \in V R W(\vec{w})$ and $\vec{u} \prec \vec{w} \backslash t\}$. If a subset $\mathcal{R}$ of $\Pi_{2}$ satisfies:

(i) for every $(t, \vec{u}) \in \Pi_{2}$ there exists $\left(t, \vec{u}_{1}\right) \in \mathcal{R}$ with $\vec{u}_{1} \prec \vec{u}$; and

(ii) for every $\left(t, \vec{u}_{1}\right) \in \mathcal{R}$ and $\vec{u}_{2} \prec \vec{u}_{1}$ we have $\left(t, \vec{u}_{2}\right) \in \mathcal{R}$,

then there exists $\vec{u} \prec \vec{w}$ such that $(t, \vec{t}) \in \mathcal{R}$ for all $t \in V R W(\vec{u})$ and $\vec{t} \prec \vec{u} \backslash t$.

Proof. Let $u_{0}=w_{1}$ and $\vec{u}_{0}=\vec{w}$. According to condition (i), there exists $\vec{u}_{1}=$ $\left(u_{n}^{1}\right)_{n \in \mathbb{N}} \in W^{\omega}(\Sigma ; v)$ such that $\vec{u}_{1} \prec \vec{w} \backslash u_{0}$ and $\left(u_{0}, \vec{u}_{1}\right) \in \mathcal{R}$. Let $u_{1}=u_{1}^{1}$. Then $\left(u_{0}, u_{1}\right) \in V R W^{<\omega}(\vec{w})$. The proof can be continued analogously to the proof of Lemma 3.4.

We are now ready to prove Theorem 3.3.

Proof of Theorem 3.3. Let $\mathcal{G} \subseteq W^{<\omega}(\Sigma), \mathcal{F} \subseteq W^{<\omega}(\Sigma ; v)$ and $\vec{w}=\left(w_{n}\right)_{n \in \mathbb{N}} \in$ $W^{\omega}(\Sigma ; v)$. For $\xi=0$ the theorem is valid, according to Carlson's theorem (Theorem 1.1). Let $\xi>0$ be a countable ordinal. Assume that the theorem is valid for every $\zeta<\xi$.

For every reduced word $s \in R W(\vec{w})$ of $\vec{w}$ over $\Sigma$ and every variable reduction $\vec{u}=\left(u_{n}\right)_{n \in \mathbb{N}} \prec \vec{w} \backslash s$ of $\vec{w} \backslash s$ over $\Sigma$ the variable reduction $\vec{u}_{s}=\left(s * u_{1}, u_{2}, \ldots\right) \prec \vec{w}$ of $\vec{w}$ over $\Sigma$ is defined. So, we can define the following set:

$\mathcal{R}_{1}=\{(s, \vec{u}): s \in R W(\vec{w}), \vec{u} \prec \vec{w} \backslash s$ and either $W^{\xi}(\Sigma)(s) \cap R W^{<\omega}\left(\vec{u}_{s}\right) \subseteq\left(\mathcal{G} \cap R W^{<\omega}(\vec{w})\right)(s)$, or $\left.W^{\xi}(\Sigma)(s) \cap R W^{<\omega}\left(\vec{u}_{s}\right) \subseteq W^{<\omega}(\Sigma) \backslash\left(\mathcal{G} \cap R W^{<\omega}(\vec{w})\right)(s)\right\}$.

Of course $\mathcal{R}_{1} \subseteq \Pi_{1}=\{(s, \vec{u}): s \in R W(\vec{w})$ and $\vec{u} \prec \vec{w} \backslash s\}$ and obviously $\mathcal{R}_{1}$ satisfies the conditions (ii) of Lemma 3.4. We will prove that $\mathcal{R}_{1}$ also satisfies condition (i) of Lemma 3.4.

Let $(s, \vec{u}) \in \Pi_{1}$. Then $s \in R W(\vec{w}) \subseteq W(\Sigma)$, hence $s=\alpha_{1} \ldots \alpha_{n-1}$ for some $n \in \mathbb{N}$ with $n>1$ and $\alpha_{1}, \ldots, \alpha_{n-1} \in \Sigma$. According to Proposition 3.2, there exists $\xi_{n}<\xi$ such that $W^{\xi}(\Sigma)(s)=W^{\xi_{n}}(\Sigma) \cap\left(W^{<\omega}(\Sigma)-s\right)$.

If $\vec{u}=\left(u_{n}\right)_{n \in \mathbb{N}} \prec \vec{w} \backslash s$, then $\vec{u}_{s}=\left(s * u_{1}, u_{2}, \ldots\right) \prec \vec{w}$. Using the induction hypothesis, there exists a variable reduction $\vec{u}^{1}=\left(u_{n}^{1}\right)_{n \in \mathbb{N}} \prec \vec{u}_{s}$ of $\vec{u}_{s}$ over $\Sigma$, such that

either $W^{\xi_{n}}(\Sigma) \cap R W^{<\omega}\left(\vec{u}^{1}\right) \subseteq\left(\mathcal{G} \cap R W^{<\omega}(\vec{w})\right)(s)$, or $\mathcal{W}^{\xi_{n}}(\Sigma) \cap R W^{<\omega}\left(\vec{u}^{1}\right) \subseteq W^{<\omega}(\Sigma) \backslash\left(\mathcal{G} \cap R W^{<\omega}(\vec{w})\right)(s)$. 
Then

either $W^{\xi}(\Sigma)(s) \cap R W^{<\omega}\left(\vec{u}^{1}\right) \subseteq\left(\mathcal{G} \cap R W^{<\omega}(\vec{w})\right)(s)$, or $W^{\xi}(\Sigma)(s) \cap R W^{<\omega}\left(\vec{u}^{1}\right) \subseteq W^{<\omega}(\Sigma) \backslash\left(\mathcal{G} \cap R W^{<\omega}(\vec{w})\right)(s)$.

Since $\vec{u}^{1}=\left(u_{n}^{1}\right)_{n \in \mathbb{N}} \prec \vec{u}_{s}$, we have that $s \propto u_{1}^{1}$, so we set $\vec{u}_{1}=\left(u_{1}^{1}-s, u_{2}^{1}, \ldots\right)$. Then $\vec{u}_{1} \prec \vec{u} \prec \vec{w} \backslash s$ and $\left(\vec{u}_{1}\right)_{s}=\vec{u}^{1}$. Thus $\left(s, \vec{u}_{1}\right) \in \mathcal{R}_{1}$. Hence, $\mathcal{R}_{1}$ satisfies condition (i) of Lemma 3.4.

According to Lemma 3.4 , there exists $\vec{w}_{1}=\left(w_{n}^{1}\right)_{n \in \mathbb{N}} \prec \vec{w}$ such that $(s, \vec{s}) \in \mathcal{R}_{1}$ for all $s \in R W\left(\vec{w}_{1}\right)$ and $\vec{s} \prec \vec{w}_{1} \backslash s$. Thus, for every $s \in R W\left(\vec{w}_{1}\right)$ and $\vec{v}=\left(v_{n}\right)_{n \in \mathbb{N}} \prec$ $\vec{w}_{1}-s$, setting $\vec{v}_{1}=\left(v_{1}-s, v_{2}, \ldots\right)$, we have that $\left(s, \vec{v}_{1}\right) \in \mathcal{R}_{1}$ and, since $\left(\vec{v}_{1}\right)_{s}=\vec{v}$, we have that

$$
\begin{aligned}
& \text { either } W^{\xi}(\Sigma)(s) \cap R W^{<\omega}(\vec{v}) \subseteq\left(\mathcal{G} \cap R W^{<\omega}(\vec{w})\right)(s) \text {, } \\
& \text { or } W^{\xi}(\Sigma)(s) \cap R W^{<\omega}(\vec{v}) \subseteq W^{<\omega}(\Sigma) \backslash\left(\mathcal{G} \cap R W^{<\omega}(\vec{w})\right)(s) .
\end{aligned}
$$

Now, defining analogously for every variable reduced word $t \in V R W\left(\vec{w}_{1}\right)$ of $\vec{w}_{1}$ over $\Sigma$ and every variable reduction $\vec{u}=\left(u_{n}\right)_{n \in \mathbb{N}} \prec \vec{w}_{1} \backslash t$ of $\vec{w}_{1} \backslash t$ over $\Sigma$ the variable reduction $\vec{u}_{t}=\left(t * u_{1}, u_{2}, \ldots\right) \prec \vec{w}_{1}$ of $\vec{w}_{1}$ over $\Sigma$, we can define the set

$\mathcal{R}_{2}=\left\{(t, \vec{u}): t \in V R W\left(\vec{w}_{1}\right), \vec{u} \prec \vec{w}_{1} \backslash t\right.$ and

either $W^{\xi}(\Sigma ; v)(t) \cap V R W^{<\omega}\left(\vec{u}_{t}\right) \subseteq\left(\mathcal{F} \cap V R W^{<\omega}(\vec{w})\right)(t)$,

or $\left.W^{\xi}(\Sigma ; v)(t) \cap V R W^{<\omega}\left(\vec{u}_{t}\right) \subseteq W^{<\omega}(\Sigma ; v) \backslash\left(\mathcal{F} \cap V R W^{<\omega}(\vec{w})\right)(t)\right\}$.

Then $\mathcal{R}_{2} \subseteq \Pi_{2}=\left\{(t, \vec{u}): t \in V R W\left(\vec{w}_{1}\right)\right.$ and $\left.\vec{u} \prec \vec{w}_{1} \backslash t\right\}$ and $\mathcal{R}_{2}$ satisfies the condition (ii) of Lemma 3.5.

Let $(t, \vec{u}) \in \Pi_{2}$. Then $t \in V R W\left(\vec{w}_{1}\right)$ and let $t=\beta_{1} \ldots \beta_{n-1} \in W(\Sigma ; v)$ for some $n \in \mathbb{N}$ with $n>1$ and $\beta_{1}, \ldots, \beta_{n-1} \in \Sigma \cup\{v\}$. According to Proposition 3.2, there exists $\xi_{n}<\xi$ such that $W^{\xi}(\Sigma ; v)(t)=W^{\xi_{n}}(\Sigma ; v) \cap\left(W^{<\omega}(\Sigma ; v)-t\right)$.

If $\vec{u}=\left(u_{n}\right)_{n \in \mathbb{N}} \prec \vec{w}_{1} \backslash t$, then $\vec{u}_{t}=\left(t * u_{1}, u_{2}, \ldots\right) \prec \vec{w}_{1}$. Using the induction hypothesis, there exists a variable reduction $\vec{u}^{1}=\left(u_{n}^{1}\right)_{n \in \mathbb{N}} \prec \vec{u}_{t}$ of $\vec{u}_{t}$ over $\Sigma$ with $t \propto u_{1}^{1}$ such that

$$
\begin{aligned}
& \text { either } W^{\xi_{n}}(\Sigma ; v) \cap V R W^{<\omega}\left(\vec{u}^{1}\right) \subseteq\left(\mathcal{F} \cap V R W^{<\omega}(\vec{w})\right)(t), \\
& \text { or } W^{\xi_{n}}(\Sigma ; v) \cap V R W^{<\omega}\left(\vec{u}^{1}\right) \subseteq W^{<\omega}(\Sigma ; v) \backslash\left(\mathcal{F} \cap V R W^{<\omega}(\vec{w})\right)(t) .
\end{aligned}
$$

Then

either $W^{\xi}(\Sigma ; v)(t) \cap V R W^{<\omega}\left(\vec{u}^{1}\right)\left(W^{<\omega}(\Sigma ; v)-t\right) \subseteq\left(\mathcal{F} \cap V R W^{<\omega}(\vec{w})\right)(t)$, or $W^{\xi}(\Sigma ; v)(t) \cap V R W^{<\omega}\left(\vec{u}^{1}\right)\left(W^{<\omega}(\Sigma ; v)-t\right)$

$$
\subseteq W^{<\omega}(\Sigma ; v) \backslash\left(\mathcal{F} \cap V R W^{<\omega}(\vec{w})\right)(t)
$$

Setting $\vec{u}_{1}=\left(u_{1}^{1}-t, u_{2}^{1}, \ldots\right)$ we have that $\vec{u}_{1} \prec \vec{u} \prec \vec{w}_{1} \backslash t$ and that $\left(t, \vec{u}_{1}\right) \in \mathcal{R}_{2}$. Hence, $\mathcal{R}_{2}$ also satisfies condition (i) of Lemma 3.5 (replacing $\vec{w}$ by $\vec{w}_{1}$ ).

According to Lemma 3.5 , there exists $\vec{w}_{2}=\left(w_{n}^{2}\right)_{n \in \mathbb{N}} \prec \vec{w}_{1} \prec \vec{w}$ such that $(t, \vec{t}) \in$ $\mathcal{R}_{2}$ for all $t \in V R W\left(\vec{w}_{2}\right)$ and $\vec{t} \prec \vec{w}_{2} \backslash t$. Hence, for every $s \in R W\left(\vec{w}_{2}\right) \subseteq R W\left(\vec{w}_{1}\right)$, $t \in V R W\left(\vec{w}_{2}\right)$ and $\vec{v}_{1} \prec \vec{w}_{2}-s \prec \vec{w}_{1}-s, \vec{v}_{2} \prec \vec{w}_{2}-t$, we have

either $W^{\xi}(\Sigma)(s) \cap R W^{<\omega}\left(\vec{v}_{1}\right) \subseteq\left(\mathcal{G} \cap R W^{<\omega}(\vec{w})\right)(s)$, or $W^{\xi}(\Sigma)(s) \cap R W^{<\omega}\left(\vec{v}_{1}\right) \subseteq W^{<\omega}(\Sigma) \backslash\left(\mathcal{G} \cap R W^{<\omega}(\vec{w})\right)(s)$; and either $W^{\xi}(\Sigma ; v)(t) \cap V R W^{<\omega}\left(\vec{v}_{2}\right) \subseteq\left(\mathcal{F} \cap V R W^{<\omega}(\vec{w})\right)(t)$, or $W^{\xi}(\Sigma ; v)(t) \cap V R W^{<\omega}\left(\vec{v}_{2}\right) \subseteq W^{<\omega}(\Sigma ; v) \backslash\left(\mathcal{F} \cap V R W^{<\omega}(\vec{w})\right)(t)$.

Let

$$
\begin{aligned}
& \mathcal{G}_{1}=\left\{s \in R W\left(\vec{w}_{2}\right): W^{\xi}(\Sigma)(s) \cap R W^{<\omega}\left(\vec{w}_{2}-s\right) \subseteq\left(\mathcal{G} \cap R W^{<\omega}(\vec{w})\right)(s)\right\}, \text { and } \\
& \mathcal{F}_{1}=\left\{t \in V R W\left(\vec{w}_{2}\right): W^{\xi}(\Sigma ; v)(t) \cap V R W^{<\omega}\left(\vec{w}_{2}-t\right) \subseteq\left(\mathcal{F} \cap V R W^{<\omega}(\vec{w})\right)(t)\right\} .
\end{aligned}
$$

We use the induction hypothesis for $\xi=0$ (Theorem 1.1). Then, there exists a 
variable reduction $\vec{u} \prec \vec{w}_{2}$ of $\vec{w}_{2}$ such that:

either $R W(\vec{u}) \subseteq \mathcal{G}_{1}$, or $R W(\vec{u}) \subseteq W(\Sigma) \backslash \mathcal{G}_{1}$; and,

either $V R W(\vec{u}) \subseteq \mathcal{F}_{1}$, or $V R W(\vec{u}) \subseteq W(\Sigma ; v) \backslash \mathcal{F}_{1}$.

Since $\vec{u} \prec \vec{w}_{2}$, we have that $R W(\vec{u}) \subseteq R W\left(\vec{w}_{2}\right)$ and $V R W(\vec{u}) \subseteq V R W\left(\vec{w}_{2}\right)$. Thus either $W^{\xi}(\Sigma)(s) \cap R W^{<\omega}(\vec{u}-s) \subseteq\left(\mathcal{G} \cap R W^{<\omega}(\vec{w})\right)(s)$ for every $s \in R W(\vec{u})$, or $W^{\xi}(\Sigma)(s) \cap R W^{<\omega}(\vec{u}-s) \subseteq W^{<\omega}(\Sigma) \backslash\left(\mathcal{G} \cap R W^{<\omega}(\vec{w})\right)(s)$ for every $s \in R W(\vec{u})$; and,

either $W^{\xi}(\Sigma ; v)(t) \cap V R W^{<\omega}(\vec{u}-t) \subseteq\left(\mathcal{F} \cap V R W^{<\omega}(\vec{w})\right)(t)$ for every $t \in$ $\operatorname{VRW}(\vec{u})$,

or $W^{\xi}(\Sigma ; v)(t) \cap V R W^{<\omega}(\vec{u}-t) \subseteq W^{<\omega}(\Sigma ; v) \backslash\left(\mathcal{F} \cap V R W^{<\omega}(\vec{w})\right)(t)$ for every $t \in V R W(\vec{u})$.

Hence,

either $W^{\xi}(\Sigma) \cap R W^{<\omega}(\vec{u}) \subseteq \mathcal{G}$, or $W^{\xi}(\Sigma) \cap R W^{<\omega}(\vec{u}) \subseteq W^{<\omega}(\Sigma) \backslash \mathcal{G}$; and, $\mathcal{F}$.

either $W^{\xi}(\Sigma ; v) \cap V R W^{<\omega}(\vec{u}) \subseteq \mathcal{F}$, or $W^{\xi}(\Sigma ; v) \cap V R W^{<\omega}(\vec{u}) \subseteq W^{<\omega}(\Sigma ; v) \backslash$

We next give a more general statement of Theorem 3.3.

Theorem 3.6. Let $\xi$ be a countable ordinal, and $\vec{w}_{0} \in \mathcal{W}^{\omega}(\Sigma ; v)$ be an infinite sequence of variable words over a finite, non-empty alphabet $\Sigma$. For any finite colorings $\chi_{1}: R W^{\xi}\left(\vec{w}_{0}\right) \rightarrow\left\{1, \ldots, r_{1}\right\}$ and $\chi_{2}: V R W^{\xi}\left(\vec{w}_{0}\right) \rightarrow\left\{1, \ldots, r_{2}\right\}$ of the sets $R W^{\xi}\left(\vec{w}_{0}\right)$ and $V R W^{\xi}\left(\vec{w}_{0}\right)$ respectively and any variable reduction $\vec{w} \prec \vec{w}_{0}$ of $\vec{w}_{0}$ over $\Sigma$, there exists a variable reduction $\vec{u} \prec \vec{w}$ of $\vec{w}$ over $\Sigma$ such that all the finite reductions of $\vec{w}$ over $\Sigma$ in the set $R W^{\xi}\left(\vec{w}_{0}\right)$ are monochromatic under $\chi_{1}$ and all the finite variable reductions of $\vec{w}$ over $\Sigma$ in the set $V R W^{\xi}\left(\vec{w}_{0}\right)$ are monochromatic under $\chi_{2}$.

Proof. Let $f: W^{<\omega}(\Sigma \cup\{v\}) \rightarrow R W^{<\omega}\left(\vec{w}_{0}\right) \cup V R W^{<\omega}\left(\vec{w}_{0}\right)$ with $f(\mathbf{s})=\vec{w}_{0}[\mathbf{s}]$. Given the finite colorings $\chi_{1}: R W^{\xi}\left(\vec{w}_{0}\right) \rightarrow\left\{1, \ldots, r_{1}\right\}$ and $\chi_{2}: V R W^{\xi}\left(\vec{w}_{0}\right) \rightarrow$ $\left\{1, \ldots, r_{2}\right\}$, are defined the finite colorings $\psi_{1}: W^{<\omega}(\Sigma) \rightarrow\left\{1, \ldots, r_{1}\right\}$ with $\psi_{1}(\mathbf{s})=$ $\chi_{1}(f(\mathbf{s}))$ in case $\mathbf{s} \in W^{\xi}(\Sigma)$ and $\psi_{1}(\mathbf{s})=1$ otherwise and $\psi_{2}: W^{<\omega}(\Sigma ; v) \rightarrow$ $\left\{1, \ldots, r_{2}\right\}$ with $\psi_{2}(\mathbf{t})=\chi_{2}(f(\mathbf{t}))$ in case $\mathbf{t} \in W^{\xi}(\Sigma ; v)$ and $\psi_{2}(\mathbf{t})=1$ otherwise.

For a given $\vec{w} \prec \vec{w}_{0}$ there exists $\vec{t} \in \mathcal{W}^{\omega}(\Sigma ; v)$ such that $\vec{w}=\vec{w}_{0}[\vec{t}]$. According to Theorem 3.3, there exists a variable reduction $\vec{t}_{1} \prec \vec{t}$ of $\vec{t}$ over $\Sigma$ such that the set $W^{\xi}(\Sigma) \cap R W^{<\omega}\left(\vec{t}_{1}\right)$ is monochromatic under $\psi_{1}$ and the set $W^{\xi}(\Sigma ; v) \cap$ $V R W^{<\omega}\left(\vec{t}_{1}\right)$ is monochromatic under $\psi_{2}$. Set $\vec{u}=\vec{w}_{0}\left[\vec{t}_{1}\right] \prec \vec{w}$. Then the set $R W^{\xi}\left(\vec{w}_{0}\right) \cap R W^{<\omega}(\vec{u})$ is monochromatic under $\chi_{1}$ and $V R W^{\xi}\left(\vec{w}_{0}\right) \cap V R W^{<\omega}(\vec{u})$ is monochromatic under $\chi_{2}$.

We recall that in case $\vec{w}_{0}=\vec{e}=\left(e_{n}\right)_{n \in \mathbb{N}}$ with $e_{n}=v$ for every $n \in \mathbb{N}$ all the infinite sequences of variable words over $\Sigma$ are variable reductions of $\vec{w}_{0}$ over $\Sigma$ and that $R W^{\xi}\left(\vec{w}_{0}\right)=W^{\xi}(\Sigma), V R W^{\xi}\left(\vec{w}_{0}\right)=W^{\xi}(\Sigma ; v)$ for every $0 \leq \xi<\omega_{1}$. In this case Theorem 3.6 is identified with Theorem A referred to in the Introduction.

For $k \in \mathbb{N}$ and $\vec{u} \in \mathcal{W}^{\omega}(\Sigma ; v)$ we have that $W^{k}(\Sigma) \cap R W^{<\omega}(\vec{u})=R W^{k}(\vec{u})$ and $W^{k}(\Sigma ; v) \cap V R W^{<\omega}(\vec{u})=V R W^{k}(\vec{u})$; hence Theorem 3.6 in case $\xi=k \in \mathbb{N}$ implies Theorem 1.2, which essentially has been proved by Furstenberg and Katznelson in [FK] (Theorems 2.7 and 3.1).

The following theorem is a finitary consequence of Theorem 3.6. It follows from Theorem 3.6 using a compactness argument. We will need the following notation 
to state it. For a word $w=\alpha_{1} \ldots \alpha_{l}$ over an alphabet $\Sigma$ let $l$ be the length of $w$. We denote by $W_{l}(\Sigma)$ the set of all words over $\Sigma$ with length $l$. For a countable ordinal $\xi$, we denote by $W_{M}^{\xi}(\Sigma)$ the set of all finite sequences of words in $W^{\xi}(\Sigma)$ such that the sum of the lengths of their words is equal to $M$.

Theorem 3.7 (Extended Hales-Jewett theorem). For every $r, n, k \in \mathbb{N}, \Sigma$ a finite, non-empty alphabet of $k$ elements, and $\xi$ a countable ordinal, there exists $M=$ $M(r, n, k, \xi) \in \mathbb{N}$ such that for every $r$-coloring of $W_{M}^{\xi}(\Sigma)$ there exists a finite sequence $\mathbf{w}=\left(w_{1}, \ldots, w_{n}\right)$ of variable words over $\Sigma$ all of whose finite reductions over $\Sigma$ in $W_{M}^{\xi}(\Sigma)$ are monochromatic.

The classical Hales-Jewett theorem ([HJ]) is a trivial consequence of the case $\xi=0, n=1$. Since van der Waerden's theorem ([vdW]) may be obtained as a corollary of the Hales-Jewett theorem, Theorem 3.7 can be used to obtain a corresponding extension of van der Waerden's theorem.

Furstenberg and Katznelson in [FK] introduced the notion of a $k$-dimensional combinatorial subspace of $W(\Sigma)$ for $k \in \mathbb{N}$ and proved (in Theorem 3.1) a partition theorem about these combinatorial subspaces. Theorem 3.3 implies an extension of this partition theorem to every countable ordinal. Let us give the necessary notation.

Let $\Sigma$ be a finite, non-empty alphabet. A finite-dimensional combinatorial subspace $[\mathbf{w}]$ of $W(\Sigma)$ is defined by a finite sequence $\mathbf{w}=\left(w_{1}, \ldots, w_{k}\right) \in \mathcal{W}^{<\omega}(\Sigma ; v)$ of variable words over $\Sigma$ as follows:

$$
[\mathbf{w}]=R W\left(\left(w_{1}, \ldots, w_{k}\right)\right)=\left\{w_{1}\left(\alpha_{1}\right) * \ldots * w_{k}\left(\alpha_{k}\right): \alpha_{1}, \ldots, \alpha_{k} \in \Sigma\right\} .
$$

In the same way, an infinite-dimensional combinatorial subspace $[\vec{w}]$ of $W(\Sigma)$ is defined by an infinite sequence $\vec{w}=\left\{\left(w_{n}\right)_{n \in \mathbb{N}} \in \mathcal{W}^{\omega}(\Sigma ; v)\right\}$ as follows:

$$
[\vec{w}]=R W(\vec{w})=\left\{w_{1}\left(\alpha_{1}\right) * \ldots * w_{k}\left(\alpha_{k}\right): k \in \mathbb{N}, \alpha_{1}, \ldots, \alpha_{k} \in \Sigma\right\} .
$$

A finite (or infinite)-dimensional combinatorial subspace of $W(\Sigma)$ contained in an infinite-dimensional combinatorial subspace $[\vec{w}]$ of $W(\Sigma)$ is called a finite (or infinite)-dimensional combinatorial subspace of $[\vec{w}]$. It is not hard to check that a finite-dimensional combinatorial subspace of $[\vec{w}]$ is of the form $[\mathbf{u}]$, where $\mathbf{u} \in$ $V R W^{<\omega}(\vec{w})$ and that an infinite-dimensional combinatorial subspace of $[\vec{w}]$ is of the form $[\vec{u}]$, where $\vec{u} \in V R W^{\omega}(\vec{w})$.

Definition 3.8. Let $\xi$ be a countable ordinal. A $\xi$-combinatorial subspace $[\mathbf{w}]$ of $W(\Sigma)$ is a finite-dimensional combinatorial subspace of $W(\Sigma)$ such that $\mathbf{w} \in$ $W^{\xi}(\Sigma ; v)$, and a $\xi$-combinatorial subspace $[\mathbf{w}]$ of an infinite-dimensional combinatorial subspace $[\vec{w}]$ of $W(\Sigma)$ is a finite-dimensional combinatorial subspace of $[\vec{w}]$ such that $\mathbf{w} \in V R W^{\xi}(\vec{w})$.

The class of $k$-combinatorial subspaces of $W(\Sigma)$, for $k \in \mathbb{N}$, coincides with the class of $k+1$-dimensional combinatorial subspaces of $W(\Sigma)$, while the class of $\xi$-combinatorial subspaces of $W(\Sigma)$, for a countable ordinal $\xi \geq \omega$, contains finitedimensional combinatorial subspaces of $W(\Sigma)$ of arbitrary large finite dimensions. Also, observe that although for $k \in \mathbb{N}$ the $k$-combinatorial subspaces of $[\vec{w}]$ are exactly the $k$-combinatorial subspaces of $W(\Sigma)$ contained in $[\vec{w}]$, for a countable ordinal $\xi \geq \omega$, it is not always true that every $\xi$-combinatorial subspace of $[\vec{w}]$ is a $\xi$-combinatorial subspace of $W(\Sigma)$, since it is not true that $d_{\vec{w}}(\mathbf{w})=d(\mathbf{w})$ for every $\mathbf{w} \in R^{<\omega}(\vec{w})$. 
We will now state a corollary of Theorem 3.3 which extends Theorem 3.1 in [FK], corresponding to finite ordinals $\xi<\omega$, to every countable ordinal $\xi$.

Corollary 3.9 (Combinatorial subspaces partition theorem). Let $\xi$ be a countable ordinal. For any finite coloring of the set $C S^{\xi}(\Sigma)$ of all $\xi$-combinatorial subspaces of $W(\Sigma)$ and any infinite-dimensional combinatorial subspace $[\vec{w}]$ of $W(\Sigma)$, there exists an infinite-dimensional combinatorial subspace $[\vec{u}]$ of $[\vec{w}]$ such that all the $\xi$-combinatorial subspaces of $W(\Sigma)$ contained in $[\vec{u}]$ are monochromatic.

Proof. Given the finite coloring $\chi: C S^{\xi}(\Sigma) \rightarrow\{1, \ldots, r\}$, we define the finite coloring $\psi: \mathcal{W}^{\xi}(\Sigma ; v) \rightarrow\{1, \ldots, r\}$ with $\psi(\mathbf{s})=\chi([\mathbf{s}])$. Apply Theorem 3.6 for $\vec{w}_{0}=\left(e_{n}\right)_{n \in \mathbb{N}}$ with $e_{n}=v$ for every $n \in \mathbb{N}$. Then for any $\vec{w} \in \mathcal{W}^{\omega}(\Sigma ; v)$, there exists a variable reduction $\vec{u}$ of $\vec{w}$ over $\Sigma$ such that all the elements of the set $W^{\xi}(\Sigma ; v) \cap V R W^{<\omega}(\vec{u})$ are $\psi$-monochromatic. Hence, for any infinite-dimensional combinatorial subspace $[\vec{w}]$ of $W(\Sigma)$, there exists an infinite-dimensional combinatorial subspace $[\vec{u}]$ of $[\vec{w}]$ such that all the $\xi$-combinatorial subspaces of $W(\Sigma)$ contained in $[\vec{u}]$ are $\chi$-monochromatic.

Corollary 3.10. Let $\xi$ be a countable ordinal, and $\vec{w}_{0} \in \mathcal{W}^{\omega}(\Sigma ; v)$. For any finite coloring of all $\xi$-combinatorial subspaces of $\left[\vec{w}_{0}\right]$ and any infinite-dimensional combinatorial subspace $[\vec{w}]$ of $\vec{w}_{0}$, there exists an infinite-dimensional combinatorial subspace $[\vec{u}]$ of $[\vec{w}]$ such that all the $\xi$-combinatorial subspaces of $\left[\vec{w}_{0}\right]$ contained in $[\vec{u}]$ are monochromatic.

Using the previous terminology, we obtain a generalization of Hales-Jewett theorem to higher dimensions, as a consequence of Theorem 3.7.

Corollary 3.11. For every $r, k \in \mathbb{N}, \Sigma$ a finite, non-empty alphabet of $k$ elements, and $\xi$ a countable ordinal, there exists $M=M(r, k, \xi) \in \mathbb{N}$ such that for any $r$-coloring of $W_{M}(\Sigma)$ there exists a monochromatic $\xi$-combinatorial subspace of $W_{M}(\Sigma)$.

\section{Basic properties of the Schreier-type families OF THE FINITE SEQUENCES OF WORDS}

This section is preparatory for the results of sections 4 and 5. Here we prove (a) the thinness of the Schreier-type families of words $W^{\xi}(\Sigma)$ and variable words $W^{\xi}(\Sigma ; v)$ (Proposition 4.2), and (b) the canonical representation of every (infinite or finite) sequence of (variable) words over $\Sigma$ with respect to the Schreier-type families (Proposition 4.3). Furthermore we introduce the (strong) Cantor-Bendixson index of a hereditary subfamily of the family of the finite sequences of (variable) words (Definition 4.10), and we prove that the index of the hereditary family generated by the $\xi$-Schreier-type family of finite sequences of words is $\xi+1$ for every countable ordinal $\xi$ (Proposition 4.12). In addition, in Theorem 4.6, we strengthen Theorem 3.3 in case the partition family is (not an arbitrary family but) a tree.

Definition 4.1. Let $\Sigma$ be a finite, non-empty alphabet and $\mathcal{F} \subseteq W^{<\omega}(\Sigma \cup\{v\})$ be a family of finite sequences of words over $\Sigma \cup\{v\}$.

(i) $\mathcal{F}$ is thin if there are no elements $\mathbf{s}=\left(s_{1}, \ldots, s_{k}\right), \mathbf{t}=\left(t_{1}, \ldots, t_{k}\right) \in \mathcal{F}$ with $\mathbf{s} \propto \mathbf{t}$ (which means that $k<l$ and $s_{i}=t_{i}$ for all $i=1, \ldots, k$ ).

(ii) $\mathcal{F}^{*}=\mathcal{F} \cup\left\{\mathbf{t} \in W^{<\omega}(\Sigma \cup\{v\}): \mathbf{t} \propto \mathbf{s}\right.$ for some $\left.\mathbf{s} \in \mathcal{F}\right\} \cup\{\emptyset\}$.

(iii) $\mathcal{F}$ is a tree if $\mathcal{F}^{*}=\mathcal{F}$. 
Proposition 4.2. Let $\vec{w}=\left(w_{n}\right)_{n \in \mathbb{N}} \in W^{\omega}(\Sigma ; v)$ be an infinite sequence of variable words over an alphabet $\Sigma$. The families $W^{\xi}(\Sigma ; v), W^{\xi}(\Sigma), V R W^{\xi}(\vec{w}), R W^{\xi}(\vec{w})$ are thin for every $\xi<\omega_{1}$.

Proof. It follows from the fact that the families $\mathcal{A}_{\xi}$ of Schreier finite subsets of $\mathbb{N}$ are thin (which means that if $s, t \in \mathcal{A}_{\xi}$ and $s$ is an initial segment of $t$, then $s=t$ ).

Proposition 4.3. Let $\xi>0$ be a countable ordinal number.

(i) Every infinite sequence $\vec{s}=\left(s_{n}\right)_{n \in \mathbb{N}} \in W^{\omega}(\Sigma \cup\{v\})$ of words over $\Sigma \cup\{v\}$ has canonical representation with respect to $W^{\xi}(\Sigma \cup\{v\})$, which means that there exists a unique strictly increasing sequence $\left(m_{n}\right)_{n \in \mathbb{N}}$ in $\mathbb{N}$ such that $\left(s_{1}, \ldots, s_{m_{1}}\right) \in$ $W^{\xi}(\Sigma \cup\{v\})$ and $\left(s^{n}, s_{m_{n-1}+1}, \ldots, s_{m_{n}}\right) \in W^{\xi}(\Sigma \cup\{v\})$ for every $n>1$, where $s^{n}=s_{1} * \ldots * s_{m_{n-1}}$.

(ii) Every non-empty finite sequence $\mathbf{s}=\left(s_{1}, \ldots, s_{k}\right) \in W^{<\omega}(\Sigma \cup\{v\})$ of words over $\Sigma \cup\{v\}$ has canonical representation with respect to $W^{\xi}(\Sigma \cup\{v\})$, which means that either $\mathbf{s} \in\left(W^{\xi}(\Sigma \cup\{v\})\right)^{*} \backslash W^{\xi}(\Sigma \cup\{v\})$ or there exist unique $n \in \mathbb{N}$, and $m_{1}, \ldots, m_{n} \in \mathbb{N}$ with $m_{1}<\ldots<m_{n} \leq k$ such that $\left(s_{1}, \ldots, s_{m_{1}}\right) \in W^{\xi}(\Sigma \cup\{v\})$, $\left(s^{n}, s_{m_{n-1}+1}, \ldots, s_{m_{n}}\right) \in W^{\xi}(\Sigma \cup\{v\})$ for every $n>1$, where $s^{n}=s_{1} * \ldots * s_{m_{n-1}}$, and in case $m_{n}<k,\left(s^{n+1}, s_{m_{n}+1}, \ldots, s_{k}\right) \in\left(W^{\xi}(\Sigma \cup\{v\})\right)^{*} \backslash W^{\xi}(\Sigma \cup\{v\})$ where $s^{n+1}=s_{1} * \ldots * s_{m_{n}}$.

Proof. (i) Let $\xi>0$ and $\vec{s}=\left(s_{n}\right)_{n \in \mathbb{N}} \in W^{\omega}(\Sigma \cup\{v\})$. Then the sequence $d\left(\left(s_{n}\right)_{n \in \mathbb{N}}\right)=\left(k_{n}\right)_{n \geq 2}$ of natural numbers has canonical representation with respect to $\mathcal{A}_{\xi}$, which means that there exists a unique strictly increasing sequence $\left(m_{n}\right)_{n \in \mathbb{N}}$ in $\mathbb{N}$ so that $\left(k_{2}, \ldots, k_{m_{1}}\right) \in \mathcal{A}_{\xi}$ and $\left(k_{m_{n-1}+1}, \ldots, k_{m_{n}}\right) \in \mathcal{A}_{\xi}$ for every $n>1$. Hence, $\left(s_{1}, \ldots, s_{m_{1}}\right) \in W^{\xi}(\Sigma \cup\{v\})$ and $\left(s^{n}, s_{m_{n-1}+1}, \ldots, s_{m_{n}}\right) \in W^{\xi}(\Sigma \cup\{v\})$ for every $n>1$, where $s^{n}=s_{1} * \ldots * s_{m_{n-1}}$.

(ii) Let $\mathbf{s}=\left(s_{1}, \ldots, s_{k}\right) \in W^{<\omega}(\Sigma \cup\{v\})$. Set $s_{n}=v$ for every $n \in \mathbb{N}$ with $n>k$. The sequence $\vec{s}=\left(s_{n}\right)_{n \in \mathbb{N}} \in W^{\omega}(\Sigma \cup\{v\})$ has canonical representation with respect to $W^{\xi}(\Sigma \cup\{v\})$, according to (i).

According to Proposition 4.3, every finite or infinite reduction (or variable reduction) of a sequence $\vec{w}=\left(w_{n}\right)_{n \in \mathbb{N}} \in W^{\omega}(\Sigma ; v)$ has canonical representation with respect to $R W^{\xi}(\vec{w})$ (or to $V R W^{\xi}(\vec{w})$ ), for every $1 \leq \xi<\omega_{1}$. For example $\mathbf{u}=\vec{w}[\mathbf{s}] \in R W^{<\omega}(\vec{w})$ has canonical representation with respect to $R W^{\xi}(\vec{w})$, as $\mathbf{s}$ has canonical representation with respect to $W^{\xi}(\Sigma)$.

Now, exploiting the canonical representation of every sequence of words over $\Sigma \cup\{v\}$ with respect to $W^{\xi}(\Sigma \cup\{v\})$, we will give alternative descriptions of the dichotomies described in Theorem 3.3.

Proposition 4.4. Let $\mathcal{G} \subseteq W^{<\omega}(\Sigma), \mathcal{F} \subseteq W^{<\omega}(\Sigma ; v)$ and let $\xi$ be a countable ordinal. Then, for every infinite sequence $\vec{u}=\left(u_{n}\right)_{n \in \mathbb{N}} \in W^{\omega}(\Sigma ; v)$ of variable words over $\Sigma$ the following are equivalent:

(i) $W^{\xi}(\Sigma) \cap R W^{<\omega}(\vec{u}) \subseteq \mathcal{G}\left(\right.$ resp. $\left.W^{\xi}(\Sigma ; v) \cap V R W^{<\omega}(\vec{u}) \subseteq \mathcal{F}\right)$.

(ii) For every variable reduction $\vec{u}_{1}$ of $\vec{u}$ the unique initial segment $\mathbf{s}=\left(u_{1}^{1}, \ldots\right.$, $\left.u_{m}^{1}\right)$ of $\vec{u}_{1}$, which is an element of $W^{\xi}(\Sigma ; v)$, satisfies the property $\left(u_{1}^{1}\left(\alpha_{1}\right), \ldots\right.$, $\left.u_{m}^{1}\left(\alpha_{m}\right)\right) \in \mathcal{G}$ for every $\alpha_{1}, \ldots, \alpha_{m} \in \Sigma($ resp. the property $\mathbf{s} \in \mathcal{F})$.

(iii) Given any sequence $\left(\vec{u}_{n}\right)_{n \in \mathbb{N}}$ of infinite sequences of variable words over $\Sigma$ such that $\vec{u}_{1} \prec \vec{u}$ and $\vec{u}_{n+1} \prec \vec{u}_{n}$ for every $n \in \mathbb{N}$ and any $t_{n} \in V R W\left(\vec{u}_{n}\right)$ with $t_{n} \propto t_{n+1}$ for every $n \in \mathbb{N}$, there exists $m \in \mathbb{N}$ such that $\left(t_{1}^{1}\left(\alpha_{1}\right), \ldots, t_{m}^{1}\left(\alpha_{m}\right)\right) \in$ 
$W^{\xi}(\Sigma) \cap \mathcal{G}$ for every $\alpha_{1}, \ldots, \alpha_{m} \in \Sigma$, where $t_{1}^{1}=t_{1}$ and $t_{i}^{1}=t_{i}-t_{i-1}$ for $i=$ $2, \ldots, m$ (resp. such that $\left.\left(t_{1}^{1}, \ldots, t_{m}^{1}\right) \in W^{\xi}(\Sigma ; v) \cap \mathcal{F}\right)$.

Proof. (i) $\Rightarrow$ (ii). Let $\vec{u}_{1}=\left(u_{n}^{1}\right)_{n \in \mathbb{N}}$ be a variable reduction of $\vec{u}$. Using the canonical representation of $\vec{u}_{1}$ with respect to $W^{\xi}(\Sigma ; v)$ (Proposition 4.3), there exists a unique initial segment $\mathbf{s}=\left(u_{1}^{1}, u_{2}^{1}, \ldots, u_{m}^{1}\right)$ of $\vec{u}_{1}$ which is an element of $W^{\xi}(\Sigma ; v)$. According to (i), $\left(u_{1}^{1}\left(\alpha_{1}\right), \ldots, u_{m}^{1}\left(\alpha_{m}\right)\right) \in W^{\xi}(\Sigma) \cap R W^{<\omega}(\vec{u}) \subseteq \mathcal{G}$ for every $\alpha_{1}, \ldots, \alpha_{m} \in \Sigma($ resp. $\mathbf{s} \in \mathcal{F})$.

(ii) $\Rightarrow$ (i). Let $\mathbf{s}=\left(s_{1}, \ldots, s_{m}\right) \in W^{\xi}(\Sigma) \cap R W^{<\omega}(\vec{u})$. There exist unique sequences $1=k_{1}<\cdots<k_{m}<k_{m+1} \in \mathbb{N}$ and $\alpha_{1}, \ldots, \alpha_{k_{m+1}-1} \in \Sigma$ such that $s_{i}=u_{k_{i}}\left(\alpha_{k_{i}}\right) * \ldots * u_{k_{i+1}-1}\left(\alpha_{k_{i+1}-1}\right)$ for all $i=1, \ldots, m$. Set $u_{i}^{1}=u_{k_{i}}(v) *$ $u_{k_{i}+1}\left(\alpha_{k_{i}+1}\right) * \ldots * u_{k_{i+1}-1}\left(\alpha_{k_{i+1}-1}\right)$ for all $i=1, \ldots, m$ and $u_{m+i}^{1}=u_{k_{m+1}-1+i}$ for every $i \in \mathbb{N}$. Then the sequence $\vec{u}_{1}=\left(u_{n}^{1}\right)_{n \in \mathbb{N}} \in W^{\omega}(\Sigma ; v)$ is a variable reduction of $\vec{u}$ and $\left(u_{1}^{1}, \ldots, u_{m}^{1}\right) \in W^{\xi}(\Sigma ; v)$. According to (ii), we have that $\mathbf{s} \in \mathcal{G}$.

If $\mathbf{t}=\left(t_{1}, \ldots, t_{m}\right) \in W^{\xi}(\Sigma ; v) \cap V R W^{<\omega}(\vec{u})$, then $\mathbf{t}$ is the unique initial segment of a variable reduction $\vec{u}_{1}$ of $\vec{u}$; hence, according to (ii), $\mathbf{t} \in \mathcal{F}$.

(ii) $\Rightarrow$ (iii). Let a sequence $\left(\vec{u}_{n}\right)_{n \in \mathbb{N}}$ of infinite sequences of variable words over $\Sigma$ be such that $\vec{u}_{1} \prec \vec{u}$ and $\vec{u}_{n+1} \prec \vec{u}_{n}$ for every $n \in \mathbb{N}$ and $t_{n} \in V R W\left(\vec{u}_{n}\right)$ with $t_{n} \propto t_{n+1}$ for every $n \in \mathbb{N}$. The sequence $\vec{t}=\left(t_{n}^{1}\right)_{n \in \mathbb{N}}$ with $t_{1}^{1}=t_{1}$ and $t_{n}^{1}=t_{n}-t_{n-1}$ for $n>1$ is a variable reduction of $\vec{u}$, hence, according to (ii), there exists $m \in \mathbb{N}$ such that $\left(t_{1}^{1}, \ldots, t_{m}^{1}\right) \in W^{\xi}(\Sigma ; v)$ and $\left(t_{1}^{1}\left(\alpha_{1}\right), \ldots, t_{m}^{1}\left(\alpha_{m}\right)\right) \in W^{\xi}(\Sigma) \cap \mathcal{G}$ for every $\alpha_{1}, \ldots, \alpha_{m} \in \Sigma$ (resp. and $\left.\left(t_{1}^{1}, \ldots, t_{m}^{1}\right) \in \mathcal{F}\right)$.

(iii) $\Rightarrow$ (ii). Let a variable reduction $\vec{u}_{1}=\left(u_{n}^{1}\right)_{n \in \mathbb{N}}$ of $\vec{u}$. Use (iii), setting $\vec{u}_{n}=\vec{u}_{1}$ for every $n \in \mathbb{N}$ and $t_{n}=u_{1}^{1} * \ldots * u_{n}^{1}$ for every $n \in \mathbb{N}$.

We will now give an alternative description for the second horn of the dichotomy proved in Theorem 3.3, in case the partition family is a tree.

Proposition 4.5. Let $\mathcal{G} \subseteq W^{<\omega}(\Sigma)$ be a tree, $\mathcal{F} \subseteq W^{<\omega}(\Sigma ; v)$ be a tree and let $\xi$ be a countable ordinal. Then

$W^{\xi}(\Sigma) \cap R W^{<\omega}(\vec{u}) \subseteq W^{<\omega}(\Sigma) \backslash \mathcal{G}$ if and only if

$\mathcal{G} \cap R W^{<\omega}(\vec{u}) \subseteq\left(W^{\xi}(\Sigma)\right)^{*} \backslash W^{\xi}(\Sigma)$, and

$W^{\xi}(\Sigma ; v) \cap V R W^{<\omega}(\vec{u}) \subseteq W^{<\omega}(\Sigma ; v) \backslash \mathcal{F}$ if and only if

$\mathcal{F} \cap V R W^{<\omega}(\vec{u}) \subseteq\left(W^{\xi}(\Sigma ; v)\right)^{*} \backslash W^{\xi}(\Sigma ; v)$.

Proof. Let $W^{\xi}(\Sigma) \cap R W^{<\omega}(\vec{u}) \subseteq W^{<\omega}(\Sigma) \backslash \mathcal{G}$ and $\mathbf{s}=\left(s_{1}, \ldots, s_{l}\right) \in \mathcal{G} \cap R W^{<\omega}(\vec{u})$. Since $\mathbf{s}$ has canonical representation with respect to $W^{\xi}(\Sigma)$ (Proposition 4.3), either $\mathbf{s} \in\left(W^{\xi}(\Sigma)\right)^{*} \backslash W^{\xi}(\Sigma)$, as required, or there exists $\mathbf{s}_{1} \in W^{\xi}(\Sigma)$ such that $\mathbf{s}_{1}=\mathbf{s}$ or $\mathbf{s}_{1} \propto \mathbf{s}$. The second case is impossible, since then $\mathbf{s}_{1} \in \mathcal{G} \cap R W^{<\omega}(\vec{u}) \cap W^{\xi}(\Sigma)$; a contradiction to our assumption. Hence, $\mathcal{G} \cap R W^{<\omega}(\vec{u}) \subseteq\left(W^{\xi}(\Sigma)\right)^{*} \backslash W^{\xi}(\Sigma)$. Obviously, $W^{\xi}(\Sigma) \cap R W^{<\omega}(\vec{u}) \subseteq W^{<\omega}(\Sigma) \backslash \mathcal{G}$ if $\mathcal{G} \cap R W^{<\omega}(\vec{u}) \subseteq\left(W^{\xi}(\Sigma)\right)^{*} \backslash W^{\xi}(\Sigma)$.

Analogously, it can be proved that $W^{\xi}(\Sigma ; v) \cap V R W^{<\omega}(\vec{u}) \subseteq W^{<\omega}(\Sigma ; v) \backslash \mathcal{F}$ if and only if $\mathcal{F} \cap V R W^{<\omega}(\vec{u}) \subseteq\left(W^{\xi}(\Sigma ; v)\right)^{*} \backslash W^{\xi}(\Sigma ; v)$.

A consequence of Proposition 4.5 is the following stronger form of Theorem 3.3 in case the partition families are trees.

Theorem 4.6. Let $\xi$ be a countable ordinal, $\Sigma$ be a finite non-empty alphabet and $\mathcal{G} \subseteq W^{<\omega}(\Sigma), \mathcal{F} \subseteq W^{<\omega}(\Sigma ; v)$ be trees. Then for every infinite sequence $\vec{w} \in W^{\omega}(\Sigma ; v)$ of variable words over $\Sigma$ there exists a variable reduction $\vec{u} \prec \vec{w}$ of 
$\vec{w}$ over $\Sigma$ such that:

either $W^{\xi}(\Sigma) \cap R W^{<\omega}(\vec{u}) \subseteq \mathcal{G}$, or $\mathcal{G} \cap R W^{<\omega}(\vec{u}) \subseteq\left(W^{\xi}(\Sigma)\right)^{*} \backslash W^{\xi}(\Sigma)$, and either $W^{\xi}(\Sigma ; v) \cap V R W^{<\omega}(\vec{u}) \subseteq \mathcal{F}$, or $\mathcal{F} \cap V R W^{<\omega}(\vec{u}) \subseteq\left(W^{\xi}(\Sigma ; v)\right)^{*} \backslash W^{\xi}(\Sigma ; v)$.

We will now define a topology on the sets $W^{<\omega}(\Sigma), W^{<\omega}(\Sigma ; v), W^{\omega}(\Sigma)$, $W^{\omega}(\Sigma ; v)$. We set $D=\{(n, \alpha): n \in \mathbb{N}, \alpha \in \Sigma \cup\{v\}\}$, which is a countable set, and we denote by $[D]^{<\omega}$ the set of all finite subsets of $D$.

Each finite sequence $\mathbf{w}$ of words over $\Sigma \cup\{v\}$ corresponds to a unique finite subset $\sigma(\mathbf{w})$ of $[D]^{<\omega}$ defined as follows: We set $\sigma(\emptyset)=\emptyset$. For $\mathbf{w}=\left(w_{1}, \ldots, w_{m}\right) \in$ $W^{<\omega}(\Sigma \cup\{v\})$, there exist $1=k_{1}<\cdots<k_{m}<k_{m+1} \in \mathbb{N}$ and $\alpha_{j} \in \Sigma \cup\{v\}$ for all $j=1, \ldots, k_{m+1}-1$ such that $w_{i}=\alpha_{k_{i}} \ldots \alpha_{k_{i+1}-1}$ for all $i=1, \ldots, m$, hence we set

$$
\sigma(\mathbf{w})=\left\{\left\{\left(j, \alpha_{j}\right): j \in \mathbb{N}, k_{i} \leq j \leq k_{i+1}-1\right\}: i \in \mathbb{N}, i \leq m\right\} .
$$

Analogously, for $\vec{w}=\left(w_{n}\right)_{n \in \mathbb{N}} \in W^{\omega}(\Sigma \cup\{v\})$, there exist $1=k_{1}<k_{2}<k_{3}<$ $\cdots \in \mathbb{N}$ and $\alpha_{j} \in \Sigma \cup\{v\}$ for every $i \in \mathbb{N}$ such that $w_{n}=\alpha_{k_{n}} \ldots \alpha_{k_{n+1}-1}$ for all $n \in \mathbb{N}$, hence we set $\sigma(\vec{w})=\left\{\left\{\left(j, \alpha_{j}\right): j \in \mathbb{N}, k_{n} \leq j \leq k_{n+1}-1\right\}: n \in \mathbb{N}\right\}$.

We identify every sequence (finite or infinite) of words over $\Sigma \cup\{v\}$ with its characteristic function in $\{0,1\}^{[D]^{<\omega}}$, via the function:

$$
I: W^{<\omega}(\Sigma \cup\{v\}) \cup W^{\omega}(\Sigma \cup\{v\}) \rightarrow\{0,1\}^{[D]^{<\omega}},
$$

with $I(\mathbf{w})=x_{\sigma(\mathbf{w})}$ for $\mathbf{w} \in W^{<\omega}(\Sigma \cup\{v\})$ and $I(\vec{w})=x_{\sigma(\vec{w})}$ for $\vec{w} \in W^{\omega}(\Sigma \cup\{v\})$,

Thus, identifying every finite sequence $\mathbf{w} \in W^{<\omega}(\Sigma \cup\{v\})$ and every infinite sequence $\vec{w} \in W^{\omega}(\Sigma \cup\{v\})$ of words over $\Sigma \cup\{v\}$ with its characteristic function $x_{\sigma(\mathbf{w})} \in\{0,1\}^{[D]^{<\omega}}$ and $x_{\sigma(\vec{w})} \in\{0,1\}^{[D]^{<\omega}}$ respectively, we topologize the sets $W^{<\omega}(\Sigma), W^{<\omega}(\Sigma ; v), W^{\omega}(\Sigma), W^{\omega}(\Sigma ; v)$ by the topology of pointwise convergence (equivalently by the relative product topology of $\{0,1\}^{[D]^{<\omega}}$ ). For example we say that a family $\mathcal{F} \subseteq W^{<\omega}(\Sigma ; v)$ is pointwise closed iff the family $\left\{x_{\sigma(\mathbf{w})}: \mathbf{w} \in \mathcal{F}\right\}$ is closed in the topology of pointwise convergence; or a family $\mathcal{U} \subseteq W^{\omega}(\Sigma)$ is pointwise closed iff $\left\{x_{\sigma(\vec{w})}: \vec{w} \in \mathcal{U}\right\}$ is pointwise closed in $\{0,1\}^{[D]^{<\omega}}$.

We next turn our attention to hereditary families of finite sequences of words.

Definition 4.7. Let $\Sigma$ be a finite, non-empty alphabet and $\mathcal{F} \subseteq W^{<\omega}(\Sigma ; v)$.

(i) $\mathcal{F}_{*}=\left\{\mathbf{t} \in W^{<\omega}(\Sigma ; v): \mathbf{t} \in V R W^{<\omega}\right.$ (s) for some $\left.\mathbf{s} \in \mathcal{F}^{*} \backslash\{\emptyset\}\right\} \cup\{\emptyset\}$.

(ii) $\mathcal{F}$ is hereditary if $\mathcal{F}_{*}=\mathcal{F}$.

Definition 4.8. Let $\Sigma$ be a finite, non-empty alphabet and $\mathcal{G} \subseteq W^{<\omega}(\Sigma)$.

(i) Set $\langle\emptyset\rangle=\emptyset$, and, for every $\mathbf{t}=\left(t_{1}, \ldots, t_{m}\right) \in W^{<\omega}(\Sigma ; v)$, set

$\langle\mathbf{t}\rangle=\left\{\left(t_{1}\left(\alpha_{1}\right), \ldots, t_{m}\left(\alpha_{m}\right)\right): \alpha_{1}, \ldots, \alpha_{m} \in \Sigma\right\}$.

(ii) $\mathcal{F}_{\mathcal{G}}=\left\{\mathbf{t}=\left(t_{1}, \ldots, t_{m}\right) \in W^{<\omega}(\Sigma ; v):\langle\mathbf{t}\rangle \subseteq \mathcal{G}\right\}$.

(ii) $\mathcal{G}_{*}=\left\{\mathbf{s}=\left(s_{1}, \ldots, s_{k}\right) \in W^{<\omega}(\Sigma): \mathbf{s} \in\langle\mathbf{t}\rangle\right.$ for some $\left.\mathbf{t} \in\left(\mathcal{F}_{\mathcal{G}}\right)_{*}\right\}$.

(iii) $\mathcal{G}$ is hereditary if $\mathcal{G}_{*}=\mathcal{G}$.

Proposition 4.9. Let $\vec{w}=\left(w_{n}\right)_{n \in \mathbb{N}} \in W^{<\omega}(\Sigma ; v)$ be an infinite sequence of variable words over $\Sigma$.

(i) If $\mathcal{G} \subseteq R W^{<\omega}(\vec{w})$ (resp. $\mathcal{F} \subseteq V R W^{<\omega}(\vec{w})$ ) is a tree, then $\mathcal{G}$ (resp. $\mathcal{F}$ ) is pointwise closed if and only if there does not exist a reduction (resp. a variable reduction) $\vec{u}=\left(u_{n}\right)_{n \in \mathbb{N}}$ of $\vec{w}$ such that $\left(u_{1}, \ldots, u_{n}\right) \in \mathcal{G}\left(\right.$ resp. $\left.\left(u_{1}, \ldots, u_{n}\right) \in \mathcal{F}\right)$ for all $n \in \mathbb{N}$.

(ii) If $\mathcal{G} \subseteq R W^{<\omega}(\vec{w})$ (resp. $\mathcal{F} \subseteq V R W^{<\omega}(\vec{w})$ ) is hereditary, then $\mathcal{G}$ (resp. $\mathcal{F})$ is pointwise closed if and only if there does not exist a variable reduction $\vec{u}$ of 
$\vec{w}$ such that $R W^{<\omega}(\vec{u}) \subseteq \mathcal{G}$ (resp. $\left.V R W^{<\omega}(\vec{u}) \subseteq \mathcal{F}\right)$. Hence, if $\mathcal{G}$ (resp. $\left.\mathcal{F}\right)$ is hereditary and pointwise closed, then every hereditary subfamily of $\mathcal{G}$ (resp. of $\mathcal{F}$ ) is also pointwise closed.

(iii) The hereditary families $\left(W^{\xi}(\Sigma) \cap R W^{<\omega}(\vec{u})\right)_{*},\left(W^{\xi}(\Sigma ; v) \cap V R W^{<\omega}(\vec{u})\right)_{*}$ and $\left(R W^{\xi}(\vec{w}) \cap R W^{<\omega}(\vec{u})\right)_{*},\left(V R W^{\xi}(\vec{w}) \cap V R W^{<\omega}(\vec{u})\right)_{*}$ are pointwise closed for every countable ordinal $\xi$ and $\vec{u} \in V R W^{\omega}(\vec{w})$.

Proof. (i) It follows from the fact that the set $R W^{<\omega}\left(\left(w_{1}, \ldots, w_{n}\right)\right)$ is finite for every $n \in \mathbb{N}$.

(ii) Let $\mathcal{G} \subseteq R W^{<\omega}(\vec{w})$ be a hereditary and not pointwise closed family. Then $\mathcal{G}_{*}=\mathcal{G}$, thus $\left(\mathcal{F}_{\mathcal{G}}\right)_{*}=\mathcal{F}_{\mathcal{G}}$. Since $\mathcal{G}$ is a tree, according to (i), there exists a reduction $\vec{u}=\left(u_{n}\right)_{n \in \mathbb{N}}$ of $\vec{w}$ such that $\left(u_{1}, \ldots, u_{n}\right) \in \mathcal{G}=\mathcal{G}_{*}$ for all $n \in \mathbb{N}$. Hence, for every $n \in \mathbb{N}$ there exist $\left(s_{1}^{n}, \ldots, s_{n}^{n}\right) \in \mathcal{F}_{\mathcal{G}} \cap V R W^{<\omega}(\vec{w})$ and $\alpha_{1}^{n}, \ldots, \alpha_{n}^{n} \in \Sigma$ such that $u_{i}=s_{i}^{n}\left(\alpha_{i}^{n}\right)$ for every $i \leq n$. Since $\Sigma$ is finite, by a compactness argument we can find a variable reduction $\vec{s}=\left(s_{n}\right)_{n \in \mathbb{N}}$ of $\vec{w}$ and $\left(\alpha_{n}\right)_{n \in \mathbb{N}} \in \Sigma$ such that $\left(s_{1}, \ldots, s_{n}\right) \in \mathcal{F}_{\mathcal{G}} \cap V R W^{<\omega}(\vec{w})$ and $u_{n}=s_{n}\left(\alpha_{n}\right)$ for all $n \in \mathbb{N}$. So, $\vec{s} \in V R W^{\omega}(\vec{w})$ and $R W^{<\omega}(\vec{s}) \subseteq \mathcal{G}$.

(iii) It follows from (ii).

For hereditary and pointwise closed families $\mathcal{G} \subseteq R W^{<\omega}(\vec{w}), \mathcal{F} \subseteq V R W^{<\omega}(\vec{w})$ for some $\vec{w} \in W^{<\omega}(\Sigma ; v)$, the strong Cantor-Bendixson index $s O_{\vec{u}}(\mathcal{G})$ of $\mathcal{G}$ and $s O_{\vec{u}}(\mathcal{F})$ of $\mathcal{F}$ can be defined with respect to every $\vec{u} \in V R W^{\omega}(\vec{w})$.

Definition 4.10. Let $\vec{w}=\left(w_{n}\right)_{n \in \mathbb{N}} \in W^{\omega}(\Sigma ; v)$ be an infinite sequence of variable words over a finite, non-empty alphabet $\Sigma$ and $\mathcal{G} \subseteq R W^{<\omega}(\vec{w}), \mathcal{F} \subseteq V R W^{<\omega}(\vec{w})$ be hereditary and pointwise closed families. For a variable reduction $\vec{u}=\left(u_{n}\right)_{n \in \mathbb{N}} \prec \vec{w}$ of $\vec{w}$ over $\Sigma$ we define the strong Cantor-Bendixson derivatives $(\mathcal{G})_{\vec{u}}^{\xi}$ of $\mathcal{G},(\mathcal{F})_{\vec{u}}^{\xi}$ of $\mathcal{F}$ on $\vec{u}$ for every $\xi<\omega_{1}$ as follows:

For every $\mathbf{s}=\left(s_{1}, \ldots, s_{k}\right) \in \mathcal{G} \cap R W^{<\omega}(\vec{u})$ and $\mathbf{t}=\left(t_{1}, \ldots, t_{k}\right) \in \mathcal{F} \cap V R W^{<\omega}(\vec{u})$ set

$$
\begin{aligned}
& A_{\mathbf{s}}^{\mathcal{G}}=\left\{w \in R W(\vec{u}): s_{1} * \ldots * s_{k} \propto w,\left(s_{1}, \ldots, s_{k}, w-\left(s_{1} * \ldots * s_{k}\right)\right) \notin \mathcal{G}\right\} ; \\
& A_{\mathbf{t}}^{\mathcal{F}}=\left\{w \in V R W(\vec{u}): t_{1} * \ldots * t_{k} \propto w,\left(t_{1}, \ldots, t_{k}, w-\left(t_{1} * \ldots * t_{k}\right)\right) \notin \mathcal{F}\right\} ; \\
& \text { and } \\
& A_{\emptyset}^{\mathcal{G}}=\{w \in R W(\vec{u}):(w) \notin \mathcal{G}\}, A_{\emptyset}^{\mathcal{F}}=\{w \in V R W(\vec{u}):(w) \notin \mathcal{F}\} .
\end{aligned}
$$

Then

$$
\begin{gathered}
(\mathcal{G})_{\vec{u}}^{0}=\left\{\mathbf{s} \in \mathcal{G} \cap R W^{<\omega}(\vec{u}): A_{\mathbf{s}}^{\mathcal{G}} \text { does not contain any sequence }\left(w_{n}\right)_{n \in \mathbb{N}}\right. \\
\text { with } \left.w_{n} \propto w_{n+1} \text { for every } n \in \mathbb{N}\right\}, \\
(\mathcal{F})_{\vec{u}}^{0}=\left\{\mathbf{t} \in \mathcal{F} \cap V R^{<\omega}(\vec{u}): A_{\mathbf{t}}^{\mathcal{F}} \text { does not contain any sequence }\left(w_{n}\right)_{n \in \mathbb{N}}\right. \\
\text { with } \left.w_{n} \propto w_{n+1} \text { for every } n \in \mathbb{N}\right\} .
\end{gathered}
$$

It is easy to verify that $(\mathcal{G})_{\vec{u}}^{0},(\mathcal{F})_{\vec{u}}^{0}$ are hereditary, hence pointwise closed (Proposition 4.9 , (ii)). So, we can define for every $\xi>0$ the $\xi$-derivatives of $\mathcal{G}$ and $\mathcal{F}$ recursively as follows:

$$
\begin{aligned}
& (\mathcal{G})_{\vec{u}}^{\zeta+1}=\left((\mathcal{G})_{\vec{u}}^{\zeta}\right)_{\vec{u}}^{0},(\mathcal{F})_{\vec{u}}^{\zeta+1}=\left((\mathcal{F})_{\vec{u}}^{\zeta}\right)_{\vec{u}}^{0} \text { for all } \zeta<\omega_{1} \text {, and } \\
& (\mathcal{G})_{\vec{u}}^{\xi}=\bigcap_{\beta<\xi}(\mathcal{G})_{\vec{u}}^{\beta},(\mathcal{F})_{\vec{u}}^{\xi}=\bigcap_{\beta<\xi}(\mathcal{F})_{\vec{u}}^{\beta} \text { for } \xi \text { a limit ordinal. }
\end{aligned}
$$


The strong Cantor-Bendixson index $s O_{\vec{u}}(\mathcal{G})$ of $\mathcal{G}$ on $\vec{u}$ is the smallest countable ordinal $\xi$ such that $(\mathcal{G})_{\vec{u}}^{\xi}=\emptyset$ and respectively the strong Cantor-Bendixson index $s O_{\vec{u}}(\mathcal{F})$ of $\mathcal{F}$ on $\vec{u}$ is the smallest countable ordinal $\xi$ such that $(\mathcal{F})_{\vec{u}}^{\xi}=\emptyset$.

Remark 4.11. Let $\vec{w}=\left(w_{n}\right)_{n \in \mathbb{N}} \in W^{\omega}(\Sigma ; v)$ and $\mathcal{G} \subseteq R W^{<\omega}(\vec{w}), \mathcal{F} \subseteq V R W^{<\omega}(\vec{w})$ be hereditary and pointwise closed families.

(i) The strong Cantor-Bendixson index $s O_{\vec{u}}(\mathcal{G})$ and also the index $s O_{\vec{u}}(\mathcal{F})$ on a variable reduction $\vec{u} \prec \vec{w}$ of $\vec{w}$ over $\Sigma$ is a countable successor ordinal less than or equal to the "usual" Cantor-Bendixson index $O(\mathcal{G})$ of $\mathcal{G}$ and $O(\mathcal{F})$ of $\mathcal{F}$ respectively into $\{0,1\}^{[D]^{<\omega}}$ (see $\left.[\mathrm{K}]\right)$.

(ii) $s O_{\vec{u}}\left(\mathcal{G} \cap R W^{<\omega}(\vec{u})\right)=s O_{\vec{u}}(\mathcal{G})$ and $s O_{\vec{u}}\left(\mathcal{F} \cap V R W^{<\omega}(\vec{u})\right)=s O_{\vec{u}}(\mathcal{F})$.

(iii) $s O_{\vec{u}}\left(\mathcal{G}_{1}\right) \leq s O_{\vec{u}}\left(\mathcal{G}_{2}\right)$ if $\mathcal{G}_{1}, \mathcal{G}_{2} \subseteq R W^{<\omega}(\vec{w})$ are hereditary and pointwise closed families with $\mathcal{G}_{1} \subseteq \mathcal{G}_{2}$ and also $s O_{\vec{u}}\left(\mathcal{F}_{1}\right) \leq s O_{\vec{u}}\left(\mathcal{F}_{2}\right)$ if $\mathcal{F}_{1}, \mathcal{F}_{2} \subseteq V R W<\omega(\vec{w})$ are hereditary and pointwise closed families with $\mathcal{F}_{1} \subseteq \mathcal{F}_{2}$.

(iv) If $\mathbf{s}=\left(s_{1}, \ldots, s_{k}\right) \in(\mathcal{G})_{\vec{u}}^{\xi}$ and $\vec{u}_{1} \prec \vec{u} \prec \vec{w}$, then $\emptyset \in(\mathcal{G})_{\vec{u}_{1}}^{\xi}$ and $\mathbf{s}_{1} \in(\mathcal{G})_{\vec{u}_{1}}^{\xi}$ where $\mathbf{s}_{1}=\left(t_{1}, t_{2}-t_{1}, \ldots, t_{l}-t_{l-1}\right)$ in case $\left\{s_{1}, s_{1} * s_{2}, \ldots, s_{1} * \ldots * s_{k}\right\} \cap R W\left(\vec{u}_{1}\right)=$ $\left\{t_{1}, \ldots, t_{l}\right\}$, since $R W\left(\vec{u}_{1}\right) \subseteq R W(\vec{u})$.

(v) If $\vec{u}_{1} \prec \vec{u} \prec \vec{w}$, then $s O_{\vec{u}_{1}}(\mathcal{G}) \geq s O_{\vec{u}}(\mathcal{G})$ and $s O_{\vec{u}_{1}}(\mathcal{F}) \geq s O_{\vec{u}}(\mathcal{F})$, according to (iv).

(vi) Let $\vec{u} \prec \vec{w}, \sigma(\vec{u})=\left\{u_{1}, u_{1} * u_{2}, u_{1} * u_{2} * u_{3}, \ldots\right\}$ and $\vec{u}_{1} \prec \vec{w}$. If $\sigma\left(\vec{u}_{1}\right) \backslash \sigma(\vec{u})$ is a finite set, then $s O_{\vec{u}_{1}}(\mathcal{G}) \geq s O_{\vec{u}}(\mathcal{G})$ and $s O_{\vec{u}_{1}}(\mathcal{F}) \geq s O_{\vec{u}}(\mathcal{F})$.

Proposition 4.12. Let $\vec{w}=\left(w_{n}\right)_{n \in \mathbb{N}} \in W^{\omega}(\Sigma ; v)$ be an infinite sequence of variable words over $\Sigma, \vec{u}_{1}=\left(u_{n}^{1}\right)_{n \in \mathbb{N}} \prec \vec{u}=\left(u_{n}\right)_{n \in \mathbb{N}} \prec \vec{w}$ be variable reductions of $\vec{w}$ over $\Sigma$ and $\xi \geq 0$ a countable ordinal. Then

$$
\begin{aligned}
& s O_{\vec{u}_{1}}\left(\left(W^{\xi}(\Sigma) \cap R W^{<\omega}(\vec{u})\right)_{*}\right)=s O_{\vec{u}_{1}}\left(\left(W^{\xi}(\Sigma ; v) \cap V R W^{<\omega}(\vec{u})\right)_{*}\right)=\xi+1 \text {, and } \\
& s O_{\vec{u}_{1}}\left(\left(R W^{\xi}(\vec{w}) \cap R W^{<\omega}(\vec{u})\right)_{*}\right)=s O_{\vec{u}_{1}}\left(\left(V R W^{\xi}(\vec{w}) \cap V R W^{<\omega}(\vec{u})\right)_{*}\right)=\xi+1 .
\end{aligned}
$$

Proof. We will prove only that $s O_{\vec{u}_{1}}\left(\left(V R W^{\xi}(\vec{w}) \cap V R W^{<\omega}(\vec{u})\right)_{*}\right)=\xi+1$ for every $\xi<\omega_{1}$, and we will leave the proof of the other equalities to the reader. We mention that $W^{\xi}(\Sigma)=R W^{\xi}(\vec{e})$ and $W^{\xi}(\Sigma ; v)=V R W^{\xi}(\vec{e})$ for every countable ordinal $\xi$, in case $\vec{e}=\left(e_{n}\right)_{n \in \mathbb{N}}$ with $e_{n}=v$ for every $n \in \mathbb{N}$.

For every $0<\xi<\omega_{1}$, the families $\left(V R W^{\xi}(\vec{w}) \cap V R W^{<\omega}(\vec{u})\right)_{*}$ are pointwise closed (Proposition 4.9, (iii)) and

$\left(V R W^{\xi}(\vec{w}) \cap V R W^{<\omega}(\vec{u})\right)(t)=V R W^{\xi_{n}}(\vec{w}) \cap\left(V R W^{<\omega}(\vec{u})-t\right)$ for some $\xi_{n}<\xi$, for every $t \in V R W(\vec{u})$ with $t \in V R W\left(w_{1}, \ldots, w_{n-1}\right)$ for $n \in \mathbb{N}, n>1$ (Proposition 3.2).

We will prove by induction that $\left(\left(V R W^{\xi}(\vec{w}) \cap V R W^{<\omega}(\vec{u})\right)_{*}\right)_{\vec{u}_{1}}^{\xi}=\{\emptyset\}$ for every $\xi<\omega_{1}$. Of course, $\left(V R W^{0}(\vec{w}) \cap V R W^{<\omega}(\vec{u})\right)_{*}=\{(s): s \in V R W(\vec{u})\} \cup\{\emptyset\}$. Thus we have that $\left(\left(V R W^{0}(\vec{w}) \cap V R W^{<\omega}(\vec{u})\right)_{*}\right)_{\vec{u}_{1}}^{0}=\{\emptyset\}$.

Let $\xi>0$ and assume that $\left(\left(V R W^{\zeta}(\vec{w}) \cap V R W^{<\omega}(\vec{u})\right)_{*}\right)_{\vec{u}_{1}}^{\zeta}=\{\emptyset\}$ for every $\zeta<\xi$ and $\vec{u}_{1} \prec \vec{u}$. So, if $\vec{u}_{1} \prec \vec{u}$ and $t \in V R W\left(\vec{u}_{1}\right)$ with $t \in V R W\left(w_{1}, \ldots, w_{n-1}\right)$, then $\left(\left(\left(V R W^{\xi}(\vec{w}) \cap V R W^{<\omega}(\vec{u})\right)(t)\right)_{*}\right)_{\vec{u}_{1}}^{\xi_{n}}=\left(\left(V R W^{\xi_{n}}(\vec{w}) \cap\left(V R W^{<\omega}(\vec{u})-t\right)\right)_{*}\right)_{\vec{u}_{1}}^{\xi_{n}}=$ $\{\emptyset\}$. This gives that $(t) \in\left(\left(V R W^{\xi}(\vec{w}) \cap V R W^{<\omega}(\vec{u})\right)_{*}\right)_{\vec{u}_{1}}^{\xi_{n}}$. So, in case $\xi=\zeta+1$ is a successor ordinal, we have that $(t) \in\left(\left(V R W^{\xi}(\vec{w}) \cap V R W^{<\omega}(\vec{u})\right)_{*}\right)_{\vec{u}_{1}}^{\zeta}$ for every $t \in V R W\left(\vec{u}_{1}\right)$, hence $\emptyset \in\left(\left(V R W^{\xi}(\vec{w}) \cap V R W^{<\omega}(\vec{u})\right)_{*}\right)_{\vec{u}_{1}}^{\xi}$. In case $\xi$ is a limit 
ordinal, we have that $\emptyset \in\left(\left(V R W^{\xi}(\vec{w}) \cap V R W^{<\omega}(\vec{u})\right)_{*}\right)_{\vec{u}_{1}}^{\xi}$, since $\emptyset \in\left(\left(V R W^{\xi}(\vec{w}) \cap\right.\right.$ $\left.\left.V R W^{<\omega}(\vec{u})\right)_{*}\right)_{\vec{u}_{1}}^{\xi_{n}}$ for every $n \in \mathbb{N}$ and $\sup \xi_{n}=\xi$.

If $\{\emptyset\} \neq\left(\left(V R W^{\xi}(\vec{w}) \cap V R W^{<\omega}(\vec{u})\right)_{*}\right)_{\vec{u}_{1}}^{\xi}$ for some $\vec{u}_{1} \prec \vec{u}$, then there exist $\vec{u}_{2} \prec \vec{u}_{1}$ and $t \in V R W\left(\vec{u}_{2}\right)$ such that $\left(\left(\left(V R W^{\xi}(\vec{w}) \cap V R W^{<\omega}(\vec{u})\right)(t)\right)_{*}\right)_{\vec{u}_{2}}^{\xi}=$ $\left(\left(V R W^{\xi_{n}}(\vec{w}) \cap\left(V R W^{<\omega}(\vec{u})-t\right)\right)_{*}\right)_{\vec{u}_{2}}^{\xi} \neq\{\emptyset\}$ (see Lemma 2.8 in [F3]). This is a contradiction to the induction hypothesis. Hence, $\{\emptyset\} \neq\left(\left(V R W^{\xi}(\vec{w}) \cap\right.\right.$ $\left.\left.V R W^{<\omega}(\vec{u})\right)_{*}\right)_{\vec{u}_{1}}^{\xi}$ and $s O_{\vec{u}_{1}}\left(\left(V R W^{\xi}(\vec{w}) \cap V R W^{<\omega}(\vec{u})\right)_{*}\right)=\xi+1$ for every $\xi<\omega_{1}$ and $\vec{u}_{1} \prec \vec{u} \prec \vec{w}$.

Corollary 4.13. For every $\vec{w}=\left(w_{n}\right)_{n \in \mathbb{N}} \in W^{\omega}(\Sigma ; v)$ and countable ordinals $\xi_{1}, \xi_{2}$ with $\xi_{1}<\xi_{2}$ there exists a variable reduction $\vec{u} \prec \vec{w}$ of $\vec{w}$ over $\Sigma$ such that:

$$
\begin{gathered}
\left(W^{\xi_{1}}(\Sigma)\right)_{*} \cap R W^{<\omega}(\vec{u}) \subseteq\left(W^{\xi_{2}}(\Sigma)\right)^{*} \backslash W^{\xi_{2}}(\Sigma), \text { and } \\
\left(W^{\xi_{1}}(\Sigma ; v)\right)_{*} \cap V R W^{<\omega}(\vec{u}) \subseteq\left(W^{\xi_{2}}(\Sigma ; v)\right)^{*} \backslash W^{\xi_{2}}(\Sigma ; v) .
\end{gathered}
$$

Proof. Of course $\left(W^{\xi_{1}}(\Sigma)\right)_{*} \subseteq W^{<\omega}(\Sigma)$ and $\left(W^{\xi_{1}}(\Sigma ; v)\right)_{*} \subseteq W^{<\omega}(\Sigma ; v)$ are trees. According to Theorem 4.6, for every infinite sequence $\vec{w} \in W^{\omega}(\Sigma ; v)$ there exists a variable reduction $\vec{u} \prec \vec{w}$ of $\vec{w}$ over $\Sigma$ such that:

either $W^{\xi_{2}}(\Sigma) \cap R W^{<\omega}(\vec{u}) \subseteq\left(W^{\xi_{1}}(\Sigma)\right)_{*}$, or $\left(W^{\xi_{1}}(\Sigma)\right)_{*} \cap R W^{<\omega}(\vec{u}) \subseteq\left(W^{\xi_{2}}(\Sigma)\right)^{*} \backslash W^{\xi_{2}}(\Sigma)$; and,

either $W^{\xi_{2}}(\Sigma ; v) \cap V R W^{<\omega}(\vec{u}) \subseteq\left(W^{\xi_{1}}(\Sigma ; v)\right)_{*}$,

or $\left(W^{\xi_{1}}(\Sigma ; v)\right)_{*} \cap V R W^{<\omega}(\vec{u}) \subseteq\left(W^{\xi_{2}}(\Sigma ; v)\right)^{*} \backslash W^{\xi_{2}}(\Sigma ; v)$.

The first alternative in each of the two dichotomies is impossible, since, otherwise, according to Proposition 4.12,

$$
\begin{aligned}
& \xi_{2}+1=s O_{\vec{u}}\left(\left(W^{\xi_{2}}(\Sigma) \cap R W^{<\omega}(\vec{u})\right)_{*}\right) \leq s O_{\vec{u}}\left(\left(W^{\xi_{1}}(\Sigma)\right)_{*}\right)=\xi_{1}+1 \text { or } \\
& \xi_{2}+1=s O_{\vec{u}}\left(\left(W^{\xi_{2}}(\Sigma ; v) \cap V R W^{<\omega}(\vec{u})\right)_{*}\right) \leq s O_{\vec{u}}\left(\left(W^{\xi_{1}}(\Sigma ; v)\right)_{*}\right)=\xi_{1}+1 ; \mathrm{a}
\end{aligned}
$$
contradiction.

\section{Schreier-type extension of Carlson's Nash-Williams type PARTITION THEOREM FOR WORDS}

According to the partition theorem on Schreier families proved in Section 2, for every countable ordinal $\xi$, every non-empty, finite alphabet $\Sigma$ and every partition $\mathcal{G}$ of the set $W^{<\omega}(\Sigma)$ of all the finite sequences of words over $\Sigma$, there exists an infinite sequence $\vec{u}$ of variable words over $\Sigma$, all of whose finite reductions in the Schreier family $W^{\xi}(\Sigma)$ are either in the partition family $\mathcal{G}$ itself or in the complement $W^{<\omega}(\Sigma) \backslash \mathcal{G}$. However, Theorem 3.3 can naturally provide no information whatsoever on whether all these finite reductions are in $\mathcal{G}$ or in its complement $W^{<\omega}(\Sigma) \backslash \mathcal{G}$. In this section we will obtain, for a partition family $\mathcal{G}$ that is a tree, a criterion on this matter, in terms of the strong Cantor-Bendixson index of $\mathcal{G}$ : if this index is greater than $\xi+1$, all $W^{\xi}(\Sigma)$-finite reductions fall in $\mathcal{G}$, and if less than $\xi$, in $W^{<\omega}(\Sigma) \backslash \mathcal{G}$ (albeit in a weaker, non-symmetrical manner) (Theorem 5.2 and Theorem 5.4).

It will be observed that the main dichotomy of Theorem 5.2 is non-symmetric, reflecting the fact that the treeness property is assumed for the family $\mathcal{G}$ itself only, and of course not for its complement $W^{<\omega}(\Sigma) \backslash \mathcal{G}$. This type of non-symmetric dichotomy is characteristic of Nash-Williams type partition theorem; in fact, from 
Theorem 5.2 and the analogous Theorem 5.4 for variable words, we will derive in the sequel various strong forms of Nash-Williams type partition theorems for words and variable words involving the Schreier-type families of words and the CantorBendixson index (Theorem B, Corollaries 5.5, 5.7, 5.8, 5.9 ), which imply as well Carlson's infinitary partition theorem (Theorem 1.3, [C]).

In the proof of Theorem 5.2 below we use Theorem 3.3 and we also exploit the properties of the Schreier-type families $\mathcal{W}^{\xi}(\Sigma)$ for $\xi<\omega_{1}$ proved in Section 3 . Toward this purpose we introduce the following definition.

Definition 5.1. Let $\mathcal{G} \subseteq W^{<\omega}(\Sigma)$ and $\mathcal{F} \subseteq W^{<\omega}(\Sigma ; v)$. We set

(i) $\mathcal{G}_{0}=\left\{\mathbf{s} \in \mathcal{G}: \mathbf{s} \in\langle\mathbf{t}\rangle\right.$ for some $\left.\mathbf{t} \in \mathcal{F}_{\mathcal{G}}\right\}$.

(ii) $\mathcal{G}_{h}=\left\{\mathbf{s} \in \mathcal{G}_{0}\right.$ : in case $\mathbf{s} \in\langle\mathbf{t}\rangle$ for some $\mathbf{t} \in \mathcal{F}_{\mathcal{G}}$; then $\langle\mathbf{u}\rangle \subseteq \mathcal{G}$ for every $\mathbf{u} \in V R W^{<\omega}\left(\mathbf{t}_{1}\right)$ for $\left.\mathbf{t}_{1} \propto \mathbf{t}\right\} \cup\{\emptyset\}$.

(iii) $\mathcal{F}_{h}=\left\{\mathbf{t} \in \mathcal{F}: V R W^{<\omega}\left(\mathbf{t}_{1}\right) \subseteq \mathcal{F}\right.$ for every $\left.\mathbf{t}_{1} \propto \mathbf{t}\right\} \cup\{\emptyset\}$.

Of course, $\mathcal{G}_{h}, \mathcal{F}_{h}$ are the largest subfamilies of $\mathcal{G} \cup\{\emptyset\}, \mathcal{F} \cup\{\emptyset\}$ which are hereditary.

Theorem 5.2. Let $\mathcal{G} \subseteq W^{<\omega}(\Sigma)$ be a family of finite sequences of words over the finite, non-empty alphabet $\Sigma$ which is a tree and let $\vec{w} \in W^{\omega}(\Sigma ; v)$ be an infinite sequence of variable words over $\Sigma$. We have the following cases:

[Case 1] The family $\mathcal{G}_{h} \cap R W^{<\omega}(\vec{w})$ is not pointwise closed.

Then, there exists a variable reduction $\vec{u}$ of $\vec{w}$ over $\Sigma$ such that

$$
R W^{<\omega}(\vec{u}) \subseteq \mathcal{G} .
$$

[Case 2] The family $\mathcal{G}_{h} \cap R W^{<\omega}(\vec{w})$ is pointwise closed.

Then, setting

$$
\zeta_{\vec{w}}^{\mathcal{G}}=\xi_{\vec{w}}^{\mathcal{G}_{h}}=\sup \left\{s O_{\vec{u}}\left(\mathcal{G}_{h} \cap R W^{<\omega}(\vec{w})\right): \vec{u} \prec \vec{w}\right\},
$$

which is a countable ordinal, the following subcases obtain:

2(i) If $\xi+1<\zeta_{\vec{w}}^{\mathcal{G}}$, then there exists $\vec{u} \prec \vec{w}$ such that

$$
W^{\xi}(\Sigma) \cap R W^{<\omega}(\vec{u}) \subseteq \mathcal{G} ;
$$

2(ii) if $\omega_{1}>\xi+1>\xi>\zeta_{\vec{w}}^{\mathcal{G}}$, then for every $\vec{u} \prec \vec{w}$ there exists $\vec{u}_{1} \prec \vec{u}$ such that

$$
W^{\xi}(\Sigma) \cap R W^{<\omega}(\vec{u}) \subseteq W^{<\omega}(\Sigma) \backslash \mathcal{G}
$$

(equivalently $\left.\mathcal{G} \cap R W^{<\omega}(\vec{u}) \subseteq\left(W^{\xi}(\Sigma)\right)^{*} \backslash W^{\xi}(\Sigma)\right)$; and

2(iii) if $\xi+1=\zeta_{\vec{s}_{0}}^{\mathcal{G}}$ or $\xi=\zeta_{\vec{s}_{0}}^{\mathcal{G}}$, then there exists $\vec{u} \prec \vec{w}$ such that

either $W^{\xi}(\Sigma) \cap R W^{<\omega}(\vec{u}) \subseteq \mathcal{G}$, or $W^{\xi}(\Sigma) \cap R W^{<\omega}(\vec{u}) \subseteq W^{<\omega}(\Sigma) \backslash \mathcal{G}$.

Proof. [Case 1] If the hereditary family $\mathcal{G}_{h} \cap R W^{<\omega}(\vec{w})$ is not pointwise closed, then, according to Proposition 4.9, there exists $\vec{u} \prec \vec{w}$ such that

$$
R W^{<\omega}(\vec{u}) \subseteq \mathcal{G}_{h} \cap R W^{<\omega}(\vec{w}) \subseteq \mathcal{G}_{h} \subseteq \mathcal{G} .
$$

[Case 2] If the hereditary family $\mathcal{G}_{h} \cap R W^{<\omega}(\vec{w})$ is pointwise closed, then the index $\zeta_{\vec{w}}^{\mathcal{G}}=\xi_{\vec{w}}^{\mathcal{G}_{h}}$ is countable, since the "usual" Cantor-Bendixson index $O\left(\mathcal{G}_{h} \cap R W^{<\omega}(\vec{w})\right)$ of $\mathcal{G}_{h} \cap R W^{<\omega}(\vec{w})$ into $\{0,1\}^{[D]^{<\omega}}$ is countable and for every $\vec{u} \prec \vec{w}$ we have that $s O_{\vec{u}}\left(\mathcal{G}_{h} \cap R W^{<\omega}(\vec{w})\right) \leq O\left(\mathcal{G}_{h} \cap R W^{<\omega}(\vec{w})\right)$, according to Remark 4.11(i)and (ii).

2 (i) Let $\xi+1<\zeta_{\vec{w}}^{\mathcal{G}}$. Then $\xi+1<\xi_{\vec{w}}^{\mathcal{G}_{h}}$, so there exists $\vec{u}_{1} \prec \vec{w}$ such that $\xi+1<s O_{\vec{u}_{1}}\left(\mathcal{G}_{h} \cap R W^{<\omega}(\vec{w})\right)$. According to Theorem 4.6, there exists a variable 
reduction $\vec{u} \prec \vec{u}_{1}$ of $\vec{u}_{1}$ over $\Sigma$ such that

either $W^{\xi}(\Sigma) \cap R W^{<\omega}(\vec{u}) \subseteq \mathcal{G}_{h}$,

or $\mathcal{G}_{h} \cap R W^{<\omega}(\vec{u}) \subseteq\left(W^{\xi}(\Sigma)\right)^{*} \backslash W^{\xi}(\Sigma) \subseteq\left(W^{\xi}(\Sigma)\right)^{*}$.

The second alternative is impossible. Indeed, if $\mathcal{G}_{h} \cap R W^{<\omega}(\vec{u}) \subseteq\left(W^{\xi}(\Sigma)\right)^{*}$, then, according to Remark 4.11 and Proposition $4.12, \xi+1<s O_{\vec{u}_{1}}\left(\mathcal{G}_{h} \cap R W^{<\omega}(\vec{w})\right) \leq$ $s O_{\vec{u}}\left(\mathcal{G}_{h} \cap R W^{<\omega}(\vec{w})\right)=s O_{\vec{u}}\left(\mathcal{G}_{h} \cap R W^{<\omega}(\vec{u})\right)$ and $s O_{\vec{u}}\left(\mathcal{G}_{h} \cap R W^{<\omega}(\vec{u})\right) \leq$ $s O_{\vec{u}}\left(\left(W^{\xi}(\Sigma)\right)^{*}\right)=\xi+1$; a contradiction. Hence, $W^{\xi}(\Sigma) \cap R W^{<\omega}(\vec{u}) \subseteq \mathcal{G}_{h} \subseteq \mathcal{G}$.

2(ii) Let $\xi+1>\xi>\zeta_{\vec{w}}^{\mathcal{G}}$, and $\vec{u} \prec \vec{w}$. For every countable ordinal $\zeta$ with $\zeta+1>\zeta_{\vec{w}}^{\mathcal{G}}$, there exists a variable reduction $\vec{u}_{1} \prec \vec{u}$ of $\vec{u}$ over $\Sigma$ such that

$$
W^{\zeta}(\Sigma) \cap R W^{<\omega}\left(\vec{u}_{1}\right) \subseteq W^{<\omega}(\Sigma) \backslash \mathcal{G}_{h} .
$$

Indeed, according to the partition theorem on Schreier families (Theorem 3.3), there exists a variable reduction $\vec{u}_{1} \prec \vec{u}$ of $\vec{u}$ over $\Sigma$ such that

either $W^{\zeta}(\Sigma) \cap R W^{<\omega}\left(\vec{u}_{1}\right) \subseteq \mathcal{G}_{h}$, or $W^{\zeta}(\Sigma) \cap R W^{<\omega}\left(\vec{u}_{1}\right) \subseteq W^{<\omega}(\Sigma) \backslash \mathcal{G}_{h}$.

The first alternative is impossible, since if $W^{\zeta}(\Sigma) \cap R W^{<\omega}\left(\vec{u}_{1}\right) \subseteq \mathcal{G}_{h}$, then, according to Remark 4.11 and Proposition 4.12, we obtain that

$$
\zeta+1=s O_{\vec{u}_{1}}\left(\left(W^{\zeta}(\Sigma) \cap R W^{<\omega}\left(\vec{u}_{1}\right)\right)_{*}\right) \leq s O_{\vec{u}_{1}}\left(\mathcal{G}_{h} \cap R W^{<\omega}\left(\vec{u}_{1}\right)\right) \leq \xi_{\vec{w}}^{\mathcal{G}_{h}}=\zeta_{\vec{w}}^{\mathcal{G}} ;
$$

a contradiction. Hence, there exists a variable reduction $\vec{u}_{1} \prec \vec{u}$ of $\vec{u}$ over $\Sigma$ such that

$$
W^{\zeta \mathcal{G}}(\Sigma) \cap R W^{<\omega}\left(\vec{u}_{1}\right) \subseteq W^{<\omega}(\Sigma) \backslash \mathcal{G}_{h} .
$$

According to Theorem 3.3, there exists $\vec{u}_{2} \prec \vec{u}_{1}$ such that

$$
\text { either } W^{\xi}(\Sigma) \cap R W^{<\omega}\left(\vec{u}_{2}\right) \subseteq \mathcal{G} \text {, or } W^{\xi}(\Sigma) \cap R W^{<\omega}\left(\vec{u}_{2}\right) \subseteq W^{<\omega}(\Sigma) \backslash \mathcal{G} \text {. }
$$

We claim that the first alternative does not hold. Indeed, if $W^{\xi}(\Sigma) \cap R W^{<\omega}\left(\vec{u}_{2}\right) \subseteq$ $\mathcal{G}$, then $\left(W^{\xi}(\Sigma) \cap R W^{<\omega}\left(\vec{u}_{2}\right)\right)^{*} \subseteq \mathcal{G}^{*}=\mathcal{G}$. Using the canonical representation of every infinite sequence of words over $\Sigma$ with respect to $W^{\xi}(\Sigma)$ (Proposition 4.3) we have that

$$
\left(W^{\xi}(\Sigma)\right)^{*} \cap R W^{<\omega}\left(\vec{u}_{2}\right)=\left(W^{\xi}(\Sigma) \cap R W^{<\omega}\left(\vec{u}_{2}\right)\right)^{*} .
$$

Hence, $\left(W^{\xi}(\Sigma)\right)^{*} \cap R W^{<\omega}\left(\vec{u}_{2}\right) \subseteq \mathcal{G}$. Since $\xi>\zeta_{\vec{w}}^{\mathcal{G}}$, according to Corollary 4.13, there exists $\vec{u}_{3} \prec \vec{u}_{2}$ such that

$$
\left(W^{\zeta_{\vec{w}}^{\mathcal{G}}}(\Sigma)\right)_{*} \cap R W^{<\omega}\left(\vec{u}_{3}\right) \subseteq\left(W^{\xi}(\Sigma)\right)^{*} \cap R W^{<\omega}\left(\vec{u}_{2}\right) \subseteq \mathcal{G} .
$$

Thus $\left(W^{\zeta_{\vec{w}}^{\mathcal{G}}}(\Sigma)\right)_{*} \cap R W^{<\omega}\left(\vec{u}_{3}\right) \subseteq \mathcal{G}_{h}$, since $\left(W^{\zeta_{\vec{w}}^{\mathcal{G}}}(\Sigma)\right)_{*} \cap R W^{<\omega}\left(\vec{u}_{3}\right)$ is a hereditary family. This is a contradiction, since $W^{\zeta_{w}^{\mathcal{G}}}(\Sigma) \cap R W^{<\omega}\left(\vec{u}_{1}\right) \subseteq W^{<\omega}(\Sigma) \backslash \mathcal{G}_{h}$ and $\vec{u}_{3} \prec \vec{u}_{1}$. Hence, according to Proposition 4.5,

$$
W^{\xi}(\Sigma) \cap R W^{<\omega}\left(\vec{u}_{2}\right) \subseteq W^{<\omega}(\Sigma) \backslash \mathcal{G} \text {, and } \mathcal{G} \cap R W^{<\omega}\left(\vec{u}_{2}\right) \subseteq\left(W^{\xi}(\Sigma)\right)^{*} \backslash W^{\xi}(\Sigma) .
$$

2(iii) In the cases $\xi+1=\zeta_{\overrightarrow{s_{0}}}^{\mathcal{G}}$ or $\xi=\zeta_{\overrightarrow{s_{0}}}^{\mathcal{G}}$, use Theorem 3.3.

Remark 5.3. Let $\mathcal{G} \subseteq W^{<\omega}(\Sigma)$ be a tree and let $\vec{w} \in W^{\omega}(\Sigma ; v)$.

(i) That both alternatives may materialize in case $\xi+1=\zeta_{\vec{w}}^{\mathcal{G}}$ can be seen by considering two simple examples:

Set $\mathcal{F}=\left\{\mathbf{t}=\left(t_{1}<t_{2}<\cdots<t_{2 k+2}\right) \in W^{<\omega}(\Sigma ; v): k \in \mathbb{N}\right.$ and $\left.\min d(\mathbf{t})=k\right\}$ and $\mathcal{G}=\left\{\mathbf{s} \in W^{<\omega}(\Sigma): \mathbf{s} \in\langle\mathbf{t}\rangle\right.$ for some $\left.\mathbf{t} \in \mathcal{F}_{*}\right\}$. It is easy to see that the hereditary family $\mathcal{G}$ is pointwise closed (according to Proposition 4.9). Analogous 
to Proposition 4.12, it can be proved that $s O_{\vec{u}}\left(G \cap R W^{<\omega}(\vec{w})\right)=\omega+1$ for every $\vec{w} \in W^{\omega}(\Sigma ; v)$ and $\vec{u} \prec \vec{w}$. Thus, $\zeta_{\vec{w}}^{\mathcal{G}}=\xi_{\vec{w}}^{\mathcal{G}}=\omega+1$. It is now easy to verify that

$$
W^{\omega}(\Sigma) \cap R W^{<\omega}(\vec{u}) \subseteq \mathcal{G} \text { for every } \vec{u} \prec \vec{w} .
$$

Set $\mathcal{F}=\left\{\mathbf{t}=\left(t_{1}<t_{2}<\cdots<t_{k+1}\right) \in W^{<\omega}(\Sigma ; v): k \in \mathbb{N}\right.$ and $\left.\min d(\mathbf{t})=2 k\right\}$ and $\mathcal{G}=\left\{\mathbf{s} \in W^{<\omega}(\Sigma): \mathbf{s} \in\langle\mathbf{t}\rangle\right.$ for some $\left.\mathbf{t} \in \mathcal{F}_{*}\right\}$. The hereditary family $\mathcal{G}$ is pointwise closed. Setting $\vec{w}=\left(w_{n}\right)_{n \in \mathbb{N}} \in W^{\omega}(\Sigma ; v)$ with $w_{1}=v$ and $w_{n}=v * v$ for every $1<n \in \mathbb{N}$, we have that $s O_{\vec{u}}\left(\mathcal{G} \cap R W^{<\omega}(\vec{w})\right)=\omega+1$ for every $\vec{u} \prec \vec{w}$. Thus, $\zeta_{\vec{w}}^{\mathcal{G}}=\xi_{\vec{w}}^{\mathcal{G}}=\omega+1$. It is now easy to see that

$$
W^{\omega}(\Sigma) \cap R W^{<\omega}(\vec{u}) \subseteq W^{<\omega}(\Sigma) \backslash \mathcal{G} \text { for every } \vec{u} \prec \vec{w},
$$

since $\mathcal{G} \cap R W^{<\omega}(\vec{u}) \subseteq\left(W^{\omega}(\Sigma)\right)^{*} \backslash W^{\omega}(\Sigma)$.

(ii) In case the family $\mathcal{G} \subseteq W^{<\omega}(\Sigma)$ is hereditary and $\xi=\zeta_{\vec{w}}^{\mathcal{G}}=\xi_{\vec{w}}^{\mathcal{G}}<\omega_{1}$, it can be proved that for every $\vec{u} \prec \vec{w}$ there exists $\vec{u}_{1} \prec \vec{u}$ such that

$$
W^{\xi}(\Sigma) \cap R W^{<\omega}(\vec{u}) \subseteq W^{<\omega}(\Sigma) \backslash \mathcal{G} .
$$

For a partition of all the finite sequences of variable words over $\Sigma$ which is a tree, an analogous strengthened theorem holds, which in fact is a stronger form of Carlson's infinitary partition theorem (Theorem 1.3, [C]). Although, the proof of this theorem is analogous to the proof of Theorem 5.2, for completeness we will give a sketch of it.

Theorem 5.4. Let $\mathcal{F} \subseteq W^{<\omega}(\Sigma ; v)$ be a family of finite sequences of variable words over the finite, non-empty alphabet $\Sigma$ which is a tree and $\vec{w} \in W^{\omega}(\Sigma ; v)$ be an infinite sequence of variable words over $\Sigma$. We have the following cases:

[Case 1] The family $\mathcal{F}_{h} \cap V R W^{<\omega}(\vec{w})$ is not pointwise closed.

Then, there exists a variable reduction $\vec{u}$ of $\vec{w}$ over $\Sigma$ such that

$$
V R W^{<\omega}(\vec{u}) \subseteq \mathcal{F} .
$$

[Case 2] The family $\mathcal{F}_{h} \cap V R W^{<\omega}(\vec{w})$ is pointwise closed.

Then, setting

$$
\zeta_{\vec{w}}^{\mathcal{F}}=\xi_{\vec{w}}^{\mathcal{F}_{h}}=\sup \left\{s O_{\vec{u}}\left(\mathcal{F}_{h} \cap V R W^{<\omega}(\vec{w})\right): \vec{u} \prec \vec{w}\right\},
$$

which is a countable ordinal, the following subcases obtain:

2(i) If $\xi+1<\zeta_{\vec{w}}^{\mathcal{F}}$, then there exists $\vec{u} \prec \vec{w}$ such that

$$
W^{\xi}(\Sigma ; v) \cap V R W^{<\omega}(\vec{u}) \subseteq \mathcal{F} ;
$$

2(ii) if $\xi+1>\xi>\zeta_{\vec{w}}^{\mathcal{F}}$, then for every $\vec{u} \prec \vec{w}$ there exists $\vec{u}_{1} \prec \vec{u}$ such that

$$
W^{\xi}(\Sigma ; v) \cap V R W^{<\omega}(\vec{u}) \subseteq W^{<\omega}(\Sigma ; v) \backslash \mathcal{F}
$$

(equivalently $\mathcal{F} \cap V R W^{<\omega}(\vec{u}) \subseteq\left(W^{\xi}(\Sigma ; v)\right)^{*} \backslash W^{\xi}(\Sigma ; v)$ ); and

2(iii) if $\xi+1=\zeta_{\vec{s}_{0}}^{\mathcal{F}}$ or $\xi=\zeta_{\vec{s}_{0}}^{\mathcal{F}}$, then there exists $\vec{u} \prec \vec{w}$ such that

either $W^{\xi}(\Sigma ; v) \cap V R W^{<\omega}(\vec{u}) \subseteq \mathcal{F}$, or $W^{\xi}(\Sigma ; v) \cap V R W^{<\omega}(\vec{u}) \subseteq W^{<\omega}(\Sigma ; v) \backslash \mathcal{F}$.

Proof. [Case 1] If the hereditary family $\mathcal{F}_{h} \cap V R W^{<\omega}(\vec{w})$ is not pointwise closed, then there exists $\vec{u} \prec \vec{w}$ with $V R W^{<\omega}(\vec{u}) \subseteq \mathcal{F}_{h} \cap V R W^{<\omega}(\vec{w}) \subseteq \mathcal{F}$ (Proposition 4.9).

[Case 2] If the hereditary family $\mathcal{F}_{h} \cap V R W^{<\omega}(\vec{w})$ is pointwise closed, then the index $\zeta_{\vec{w}}^{\mathcal{F}}=\xi_{\vec{w}}^{\mathcal{F}_{h}}$ is countable, according to Remark 4.11(i) and (ii). 
2(i) Let $\xi+1<\zeta_{\vec{w}}^{\mathcal{F}}$. Then $\xi+1<s O_{\vec{u}_{1}}\left(\mathcal{F}_{h} \cap V R W^{<\omega}(\vec{w})\right)$ for some $\vec{u}_{1} \prec \vec{w}$. Using Theorem 4.6, Remark 4.11 and Proposition 4.12, we have that

$$
W^{\xi}(\Sigma ; v) \cap V R W^{<\omega}(\vec{u}) \subseteq \mathcal{F}_{h} \subseteq \mathcal{F} .
$$

2(ii) Let $\xi+1>\xi>\zeta_{\vec{w}}^{\mathcal{F}}$ and $\vec{u} \prec \vec{w}$. According to Theorem 3.3, Remark 4.11 and Proposition 4.12 , there exists a variable reduction $\vec{u}_{1} \prec \vec{u}$ of $\vec{u}$ over $\Sigma$ such that

$$
W^{\zeta_{\vec{w}}^{\mathcal{F}}}(\Sigma ; v) \cap V R W^{<\omega}\left(\vec{u}_{1}\right) \subseteq W^{<\omega}(\Sigma ; v) \backslash \mathcal{F}_{h}
$$

Again using Theorem 3.3, there exists $\vec{u}_{2} \prec \vec{u}_{1}$ such that

either $W^{\xi}(\Sigma ; v) \cap V R W^{<\omega}\left(\vec{u}_{2}\right) \subseteq \mathcal{F}$, or $W^{\xi}(\Sigma) \cap V R W^{<\omega}\left(\vec{u}_{2}\right) \subseteq W^{<\omega}(\Sigma) \backslash \mathcal{F}$. We claim that the first alternative does not hold. Indeed, if $W^{\xi}(\Sigma ; v) \cap V R W^{<\omega}\left(\vec{u}_{2}\right) \subseteq$ $\mathcal{F}$, then, using the canonical representation of every infinite sequence of variable words over $\Sigma$ with respect to $W^{\xi}(\Sigma ; v)$ (Proposition 4.3), it is easy to check that

$$
\left(W^{\xi}(\Sigma ; v)\right)^{*} \cap V R W^{<\omega}\left(\vec{u}_{2}\right)=\left(W^{\xi}(\Sigma ; v) \cap V R W^{<\omega}\left(\vec{u}_{2}\right)\right)^{*} \subseteq \mathcal{F}^{*}=\mathcal{F} .
$$

Since $\xi>\zeta_{\vec{w}}^{\mathcal{F}}$, according to Corollary 4.13 , there exists $\vec{u}_{3} \prec \vec{u}_{2}$ such that

$$
\left(W^{\zeta_{\vec{w}}^{\mathcal{F}}}(\Sigma ; v)\right)_{*} \cap V R W^{<\omega}\left(\vec{u}_{3}\right) \subseteq\left(W^{\xi}(\Sigma ; v)\right)^{*} \cap V R W^{<\omega}\left(\vec{u}_{2}\right) \subseteq \mathcal{F},
$$

and consequently such that $\left(W^{\zeta_{\vec{w}}^{\mathcal{F}}}(\Sigma ; v)\right)_{*} \cap V R W^{<\omega}\left(\vec{u}_{3}\right) \subseteq \mathcal{F}_{h}$. This is a contradiction.

2(iii) In the cases $\xi+1=\zeta_{\vec{w}}^{\mathcal{F}}$ or $\xi=\zeta_{\vec{w}}^{\mathcal{F}}$, use Theorem 3.3.

That both alternatives may materialize in case $\xi+1=\zeta_{\vec{w}}^{\mathcal{F}}$ can be seen by considering the following examples:

Set $\mathcal{F}=\left\{\mathbf{t}=\left(t_{1}<t_{2}<\cdots<t_{2 k+2}\right) \in W^{<\omega}(\Sigma ; v): k \in \mathbb{N}\right.$ and $\left.\min d(\mathbf{t})=k\right\}$. The hereditary family $\mathcal{F}_{*}$ is pointwise closed and $s O_{\vec{u}}\left(\mathcal{F}_{*}\right)=\omega+1$ for every $\vec{w} \in$ $\mathcal{W}^{\omega}(\Sigma ; v)$ and $\vec{u} \prec \vec{w}$. Thus, $\zeta_{\vec{w}}^{\mathcal{F}_{*}}=\xi_{\vec{w}}^{\mathcal{F}_{*}}=\omega+1$. It is now easy to verify that

$$
W^{\omega}(\Sigma ; v) \cap V R W^{<\omega}(\vec{u}) \subseteq \mathcal{F}_{*} \text { for every } \vec{u} \prec \vec{w} \text {. }
$$

Set $\mathcal{F}=\left\{\mathbf{t}=\left(t_{1}<t_{2}<\cdots<t_{k+1}\right) \in W^{<\omega}(\Sigma ; v): k \in \mathbb{N}\right.$ and $\left.\min d(\mathbf{t})=2 k\right\}$. The hereditary family $\mathcal{F}_{*}$ is pointwise closed and $s O_{\vec{u}}\left(\mathcal{F}_{*}\right)=\omega+1$ for every $\vec{u} \prec \vec{w}$, where $\vec{w}=\left(w_{n}\right)_{n \in \mathbb{N}} \in \mathcal{W}^{\omega}(\Sigma ; v)$ with $w_{1}=v$ and $w_{n}=v * v$ for every $1<n \in \mathbb{N}$. Thus, $\zeta_{\vec{w}}^{\mathcal{F}_{*}}=\xi_{\vec{w}}^{\mathcal{F}_{*}}=\omega+1$. It is now easy to see that

$$
\mathcal{F}_{*} \cap V R W^{<\omega}(\vec{u}) \subseteq\left(W^{\omega}(\Sigma ; v)\right)^{*} \backslash W^{\omega}(\Sigma ; v) \text { for every } \vec{u} \prec \vec{w} .
$$

An immediate consequence of Theorems 5.2 and 5.4 is Theorem B, referred to in the Introduction, which is a strengthened form of Theorem 3.3 in that the partitions are trees. A quite simplified consequence of Theorem 5.4, one not involving Schreier-type families of countable ordinal index, is equivalent to Carlson's infinitary partition theorem (Theorem 1.3) proved in [C].

Corollary 5.5. Let $\mathcal{F} \subseteq W^{<\omega}(\Sigma ; v)$ be a family of finite sequences of variable words over an alphabet $\Sigma$ which is a tree. Then for every infinite sequence $\vec{w} \in W^{\omega}(\Sigma ; v)$ of variable words over $\Sigma$ there exists a variable reduction $\vec{u} \prec \vec{w}$ of $\vec{w}$ over $\Sigma$ such that:

either $\operatorname{VR} W^{<\omega}(\vec{u}) \subseteq \mathcal{F}$,

or for every variable reduction $\vec{u}_{1}$ of $\vec{u}$ there exists an initial segment of $\vec{u}_{1}$ which belongs to $W^{<\omega}(\Sigma ; v) \backslash \mathcal{F}$.

Proof. The proof follows from Theorem 5.4 (case 1 and subcase 2(ii)) and Proposition 4.4 . 
Remark 5.6. Carlson's Theorem 1.3 is equivalent to Corollary 5.5. Indeed:

(i) Corollary 5.5 implies Theorem 1.3. Indeed, let $\mathcal{U} \subseteq W^{\omega}(\Sigma ; v)$ be a pointwise closed family of infinite sequences of variable words over $\Sigma$ and $\vec{w} \in W^{\omega}(\Sigma ; v)$. Set

$$
\mathcal{F}_{\mathcal{U}}=\left\{\mathbf{t}=\left(t_{1}, \ldots, t_{k}\right) \in W^{<\omega}(\Sigma ; v): k \in \mathbb{N} \text { and there exists } \vec{t} \in \mathcal{U} \text { with } \mathbf{t} \propto \vec{t}\right\} .
$$

Since the family $\mathcal{F}_{\mathcal{U}}$ is a tree, we may use Corollary 5.5. Then we have the following two cases:

[Case 1] There exists $\vec{u} \prec \vec{w}$ such that $V R W^{<\omega}(\vec{u}) \subseteq \mathcal{F}_{\mathcal{U}}$. Then, $V R W^{\omega}(\vec{u}) \subseteq \mathcal{U}$. Indeed, if $\vec{t}=\left(t_{n}\right)_{n \in \mathbb{N}} \in V R W^{\omega}(\vec{u})$, then $\left(t_{1}, \ldots, t_{k}\right) \in \mathcal{F}_{\mathcal{U}}$ for every $k \in \mathbb{N}$. Hence, for each $k \in \mathbb{N}$ there exists $\vec{t}_{k}=\left(t_{n}^{k}\right)_{n \in \mathbb{N}} \in \mathcal{U}$ such that $t_{n}^{k}=t_{n}$ for every $n \in \mathbb{N}$ with $n \leq k$. Since $\left(\vec{t}_{k}\right)_{k \in \mathbb{N}}$ converges pointwise to $\vec{t}$ and $\mathcal{U}$ is pointwise closed, we have that $\vec{t} \in \mathcal{U}$ and consequently that $V R W^{\omega}(\vec{u}) \subseteq \mathcal{U}$.

[Case 2] There exists $\vec{u} \prec \vec{w}$ such that every variable reduction $\vec{u}_{1}$ of $\vec{u}$ has an initial segment belonging to $W^{<\omega}(\Sigma ; v) \backslash \mathcal{F}_{\mathcal{U}}$. Hence, $V R W^{\omega}(\vec{u}) \subseteq W^{\omega}(\Sigma ; v) \backslash \mathcal{U}$.

(ii) Theorem 1.3 implies Corollary 5.5. Indeed, let $\mathcal{F} \subseteq W^{<\omega}(\Sigma ; v)$ which is a tree and $\vec{w} \in W^{\omega}(\Sigma ; v)$. Set

$$
\mathcal{U}_{\mathcal{F}}=\left\{\vec{t}=\left(t_{n}\right)_{n \in \mathbb{N}} \in W^{\omega}(\Sigma ; v) \text { : there exists } k \in \mathbb{N} \text { such that }\left(t_{1}, \ldots, t_{k}\right) \in \mathcal{F}\right\} .
$$

Then $\mathcal{W}^{\omega}(\Sigma ; v) \backslash \mathcal{U}_{\mathcal{F}}$ is pointwise closed, so, using Theorem 1.3 for the family $\mathcal{W}^{\omega}(\Sigma ; v) \backslash \mathcal{U}_{\mathcal{F}}$, we obtain Corollary 5.5.

In fact Corollary 5.5 holds for arbitrary partitions of $W^{<\omega}(\Sigma ; v)$, not necessarily trees; this is the content of the next result.

Corollary 5.7. Let $\mathcal{F} \subseteq W^{<\omega}(\Sigma ; v)$ be a family of finite sequences of variable words over an alphabet $\Sigma$. Then for every infinite sequence $\vec{w} \in W^{\omega}(\Sigma ; v)$ of variable words over $\Sigma$ there exists a variable reduction $\vec{u} \prec \vec{w}$ of $\vec{w}$ over $\Sigma$ such that:

either $V R W^{<\omega}(\vec{u}) \subseteq \mathcal{F}$,

or for every variable reduction $\vec{u}_{1}$ of $\vec{u}$ there exists an initial segment of $\vec{u}_{1}$ which belongs to $W^{<\omega}(\Sigma ; v) \backslash \mathcal{F}$.

Proof. Let $\mathcal{F}_{1}=\left\{\mathbf{t}=\left(t_{1}, \ldots, t_{k}\right) \in \mathcal{F}:\left(t_{1}, \ldots, t_{n}\right) \in \mathcal{F}\right.$ for all $\left.n \leq k \in \mathbb{N}\right\} \cup\{\emptyset\}$. The family $\mathcal{F}_{1}$ is a tree. According to Corollary 5.5 , there exists $\vec{u} \prec \vec{w}$ such that:

either $V R^{<\omega}(\vec{u}) \subseteq \mathcal{F}_{1} \subseteq \mathcal{F}$,

or for every variable reduction $\vec{u}_{1}$ of $\vec{u}$ there exists an initial segment of $\vec{u}_{1}$ which belongs to $\mathcal{W}^{<\omega}(\Sigma ; v) \backslash \mathcal{F}_{1}$. Let $\vec{u}_{1}=\left(u_{n}^{1}\right)_{n \in \mathbb{N}} \prec \vec{u}$, and let $k \in \mathbb{N}$ such that $\mathbf{t}=\left(u_{1}^{1}, \ldots, u_{k}^{1}\right) \in W^{<\omega}(\Sigma ; v) \backslash \mathcal{F}_{1}=\left(\mathcal{F} \backslash \mathcal{F}_{1}\right) \cup\left(W^{<\omega}(\Sigma ; v) \backslash \mathcal{F}\right)$. Then, either $\mathbf{t} \in W^{<\omega}(\Sigma ; v) \backslash \mathcal{F}$, as required, or $\mathbf{t} \in \mathcal{F} \backslash \mathcal{F}_{1}$. In case $\mathbf{t} \in \mathcal{F} \backslash \mathcal{F}_{1}$, by the definition of $\mathcal{F}_{1}$, there exists $n \in \mathbb{N}$ with $n \leq k$ such that $\left(u_{1}^{1}, \ldots, u_{n}^{1}\right) \in W^{<\omega}(\Sigma ; v) \backslash \mathcal{F}$, as required.

The result for families of (constant) words, corresponding to Corollary 5.7, can now be obtained as a corollary to Theorem 5.2.

Corollary 5.8. Let $\mathcal{G} \subseteq W^{<\omega}(\Sigma)$ be a family of finite sequences of words over the alphabet $\Sigma$. Then for every infinite sequence $\vec{w} \in W^{\omega}(\Sigma ; v)$ of variable words over $\Sigma$ there exists a variable reduction $\vec{u} \prec \vec{w}$ of $\vec{w}$ over $\Sigma$ such that:

either $R W^{<\omega}(\vec{u}) \subseteq \mathcal{G}$,

or for every reduction $\vec{u}_{1}$ of $\vec{u}$ there exists an initial segment of $\vec{u}_{1}$ which belongs to $W^{<\omega}(\Sigma) \backslash \mathcal{G}$. 
Proof. If $\mathcal{G}$ is a tree, then the proof follows from Theorem 5.2 (case 1 and subcase 2(ii)) and Proposition 4.4. If not set

$\mathcal{G}_{1}=\left\{\mathbf{s}=\left(s_{1}, \ldots, s_{l}\right) \in \mathcal{G}:\left(s_{1}, \ldots, s_{k}\right) \in \mathcal{G}\right.$ for every $k \in \mathbb{N}$ with $\left.k \leq l\right\} \cup\{\emptyset\}$. The family $\mathcal{G}_{1}$ is a tree and $\mathcal{G}_{1} \subseteq \mathcal{G}$. Hence, there exists $\vec{u} \prec \vec{w}$ such that:

either $R W^{<\omega}(\vec{u}) \subseteq \mathcal{G}_{1} \subseteq \mathcal{G}$,

or for every reduction $\vec{u}_{1}$ of $\vec{u}$ there exists an initial segment of $\vec{u}_{1}$ which belongs to $W^{<\omega}(\Sigma) \backslash \mathcal{G}_{1}$. Given that for every reduction $\vec{u}_{1}$ of $\vec{u}$ there exists an initial segment $\mathbf{s}$ of $\vec{u}_{1}$ which belongs to $W^{<\omega}(\Sigma) \backslash \mathcal{G}_{1}=\left(W^{<\omega}(\Sigma) \backslash \mathcal{G}\right) \cup\left(\mathcal{G} \backslash \mathcal{G}_{1}\right)$ we have that there exists an initial segment of of $\vec{u}_{1}$ which belongs to $W^{<\omega}(\Sigma) \backslash \mathcal{G}$, as required.

Corollary 5.8 is equivalent to the following infinitary partition theorem, which is the counterpart for (constant) words of Carlson's infinitary partition theorem (Theorem 1.3, Corollary 5.5).

Corollary 5.9. Let $\mathcal{U} \subseteq W^{\omega}(\Sigma)$ be a pointwise closed family of infinite sequences of words over a finite non-empty alphabet $\Sigma$ and $\vec{w} \in W^{\omega}(\Sigma ; v)$ be an infinite sequence of variable words. Then there exists a variable reduction $\vec{u} \prec \vec{w}$ of $\vec{w}$ over $\Sigma$, such that

$$
\text { either } R W^{\omega}(\vec{u}) \subseteq \mathcal{U}, \quad \text { or } \quad R W^{\omega}(\vec{u}) \subseteq W^{\omega}(\Sigma) \backslash \mathcal{U}
$$

\section{Schreier-type version of Carlson's EllentudK type PARTITION THEOREM FOR WORDS}

In this final section, we establish (in Theorem 6.2) a rather technical strengthening of Theorem B (mentioned in the introduction) derived from Theorem 5.4, involving the Ellentuck topology $\mathfrak{T}_{E}$, defined on $W^{\omega}(\Sigma ; v)$ (Definition 6.1). A simple consequence of Theorem 6.2 is the characterization of completely Ramsey partitions of $W^{\omega}(\Sigma ; v)$ in terms of the Baire property in the topology $\mathfrak{T}_{E}$ (Corollary 6.7), a result proved with a different method by Carlson in $[\mathrm{C}]$. A similar characterization of completely Ramsey partitions of $W^{\omega}(\Sigma)$ can be proved analogously, as a consequence of Theorem 5.2.

We start by defining the topology $\mathfrak{T}_{E}$ on $W^{\omega}(\Sigma ; v)$, an analogue of the Ellentuck topology on $[\mathbb{N}]^{\omega}$, defined in $[\mathrm{E}]$. For simplicity, we write $\emptyset \propto \vec{w}$ and $\vec{w} \backslash \emptyset=\vec{w}$ for every $\vec{w} \in W^{\omega}(\Sigma ; v)$.

Definition 6.1. Let $\mathfrak{T}_{E}$ be the topology on $W^{\omega}(\Sigma ; v)$ with basic open sets of the form $[\mathbf{s}, \vec{s}]$ for $\mathbf{s} \in W^{<\omega}(\Sigma ; v)$ and $\vec{s} \in W^{\omega}(\Sigma ; v)$, where for $\mathbf{s} \in W^{<\omega}(\Sigma ; v) \backslash\{\emptyset\}$

$[\mathbf{s}, \vec{s}]=\left\{\vec{w} \in W^{\omega}(\Sigma ; v): \mathbf{s} \propto \vec{w}\right.$ and $\left.\vec{w} \backslash \mathbf{s} \prec \vec{s}\right\}$ and $[\emptyset, \vec{s}]=\left\{\vec{w} \in W^{\omega}(\Sigma ; v): \vec{w} \prec \vec{s}\right\}$.

The topology $\mathfrak{T}_{E}$ is stronger than the relative topology of $W^{\omega}(\Sigma ; v)$ with respect to the pointwise convergence topology of $\{0,1\}^{[D]^{<\omega}}$, which has basic open sets of the form $[\mathbf{s}, \vec{e}]=\left\{\vec{w} \in W^{\omega}(\Sigma ; v): \mathbf{s} \propto \vec{w}\right\}$ for $\mathbf{s} \in W^{<\omega}(\Sigma ; v)$ and $\vec{e}=\left(e_{n}\right)_{n \in \mathbb{N}}$ with $e_{n}=v$ for every $n \in \mathbb{N}$.

We denote by $\hat{\mathcal{U}}$ and $\mathcal{U}^{\diamond}$ the closure and the interior respectively of a family $\mathcal{U} \subseteq W^{\omega}(\Sigma ; v)$ in the topology $\mathfrak{T}_{E}$. Then it is easy to see that

$$
\begin{aligned}
& \hat{\mathcal{U}}=\left\{\vec{w} \in W^{\omega}(\Sigma ; v):[\mathbf{s}, \vec{w} \backslash \mathbf{s}] \cap \mathcal{U} \neq \emptyset \text { for every } \mathbf{s} \propto \vec{w}\right\} ; \text { and } \\
& \mathcal{U}^{\diamond}=\left\{\vec{w} \in W^{\omega}(\Sigma ; v): \text { there exists } \mathbf{s} \propto \vec{w} \text { such that }[\mathbf{s}, \vec{w} \backslash \mathbf{s}] \subseteq \mathcal{U}\right\} .
\end{aligned}
$$

Now we can state the main theorem of this section. For $\mathbf{s}=\left(s_{1}, \ldots, s_{k}\right), \mathbf{t}=$ $\left(t_{1}, \ldots, t_{l}\right) \in W^{<\omega}(\Sigma ; v)$ we set $\mathbf{s} \odot \mathbf{t}=\left(s_{1}, \ldots, s_{k}, t_{1}, \ldots, t_{k}\right)$ and $\emptyset \odot \mathbf{t}=\mathbf{t} \odot \emptyset=\mathbf{t}$. 
Theorem 6.2. Let $\mathcal{U} \subseteq W^{\omega}(\Sigma ; v), \mathbf{s} \in W^{<\omega}(\Sigma ; v)$ and $\vec{s} \in W^{\omega}(\Sigma ; v)$. Then

either there exists $\vec{u} \prec \vec{s}$ such that $[\mathbf{s}, \vec{u}] \subseteq \hat{\mathcal{U}}$,

or there exists a countable ordinal $\xi_{0}=\zeta_{(\mathbf{s}, \vec{s})}^{\mathcal{U}}$ such that for every $\xi>\xi_{0}$ there exists $\vec{u} \prec \vec{s}$ with $[\mathbf{s} \odot \mathbf{t}, \vec{t} \backslash \mathbf{t}] \subseteq W^{\omega}(\Sigma ; v) \backslash \mathcal{U}$ for every $\vec{t} \prec \vec{u}$ and $\mathbf{t} \in W^{\xi}(\Sigma ; v)$ with $\mathbf{t} \propto \vec{t}$

For the proof of this theorem we will make use of the following lemma, which is analogous to Lemma 3.5.

Lemma 6.3. Let $\mathcal{R} \subseteq\left\{[\mathbf{s}, \vec{s}]: \mathbf{s} \in W^{<\omega}(\Sigma ; v), \vec{s} \in W^{\omega}(\Sigma ; v)\right\}$ with the properties:

(i) for every $(\mathbf{s}, \vec{s}) \in W^{<\omega}(\Sigma ; v) \times W^{\omega}(\Sigma ; v)$ there exists $\vec{s}_{1} \prec \vec{s}$ such that $\left[\mathbf{s}, \vec{s}_{1}\right] \in$ $\mathcal{R}$; and,

(ii) for every $\left[\mathbf{s}, \vec{s}_{1}\right] \in \mathcal{R}$ and $\vec{s}_{2} \prec \vec{s}_{1}$ we have $\left[\mathbf{s}, \vec{s}_{2}\right] \in \mathcal{R}$.

Then, for every $(\mathbf{s}, \vec{s}) \in \mathcal{W}^{<\omega}(\Sigma ; v) \times \mathcal{W}^{\omega}(\Sigma ; v)$ there exists $\vec{u} \in[\mathbf{s}, \vec{s}]$ such that $[\mathbf{s} \odot \mathbf{t}, \vec{t} \backslash \mathbf{t}] \in \mathcal{R}$ for every $\vec{t} \prec \vec{u} \backslash \mathbf{s}$ and $\mathbf{t} \propto \vec{t}$.

Proof. Let $\mathbf{s} \in \mathcal{W}^{<\omega}(\Sigma ; v)$ and $\vec{s} \in \mathcal{W}^{\omega}(\Sigma ; v)$. According to assumption (i), there exists $\vec{s}_{1}=\left(s_{n}^{1}\right)_{n \in \mathbb{N}} \prec \vec{s}$ such that $\left[\mathbf{s}, \vec{s}_{1}\right] \in \mathcal{R}$. Set $u_{1}=s_{1}^{1} \in V R W(\vec{s})$. Then, there exists $\vec{s}_{2}=\left(s_{n}^{2}\right)_{n \in \mathbb{N}} \prec \vec{s}_{1} \backslash\left(u_{1}\right)$ such that $\left[\mathbf{s} \odot\left(u_{1}\right), \vec{s}_{2}\right] \in \mathcal{R}$. Set $u_{2}=s_{1}^{2}$. We have $\left(u_{1}, u_{2}\right) \in V R W^{<\omega}(\vec{s})$ and $\vec{s}_{2} \prec \vec{s} \backslash u_{1}$.

Let $n \in \mathbb{N}, n>1$. We now assume that there have been constructed $\vec{s}_{1}, \ldots, \vec{s}_{n} \in$ $\mathcal{W}^{\omega}(\Sigma ; v)$ and $u_{1}, u_{2}, \ldots, u_{n} \in W(\Sigma ; v)$ such that $\left(u_{1}, u_{2}, \ldots, u_{n}\right) \in V R W^{<\omega}(\vec{s})$, $\vec{s}_{i+1} \prec \vec{s} \backslash u_{1} * \ldots * u_{i}, \vec{s}_{i+1} \prec \vec{s}_{i} \backslash\left(u_{i}\right)$ for every $i=1, \ldots, n-1,\left(u_{i}\right) \propto \vec{s}_{i}$ for every $i=1, \ldots, n$, and $\left[\mathbf{s} \odot \mathbf{t}, \vec{s}_{i+1}\right] \in \mathcal{R}$ for every $\mathbf{t} \in V R W^{<\omega}\left(\left(u_{1}, \ldots, u_{i}\right)\right)$ and $i=1, \ldots, n-1$.

We will construct $\vec{s}_{n+1}$ and $u_{n+1}$. Let $\left\{\mathbf{t}_{1}, \ldots, \mathbf{t}_{m}\right\}=V R W^{<\omega}\left(\left(u_{1}, \ldots, u_{n}\right)\right)$ for some $m \in \mathbb{N}$. According to assumption (i), there exist $\vec{s}_{n+1}^{1}, \ldots, \vec{s}_{n+1}^{m} \in W^{\omega}(\Sigma ; v)$ such that $\vec{s}_{n+1}^{m} \prec \cdots \prec \vec{s}_{n+1}^{1} \prec \vec{s}_{n} \backslash\left(u_{n}\right)$ and $\left[\mathbf{s} \odot \mathbf{t}_{i}, \vec{s}_{n+1}^{i}\right] \in \mathcal{R}$ for every $i=1, \ldots, m$. Let $\vec{s}_{n+1}=\vec{s}_{n+1}^{m}=\left(s_{i}^{n+1}\right)_{i \in \mathbb{N}}$ and $u_{n+1}=s_{1}^{n+1}$. Of course $\vec{s}_{n+1} \prec \vec{s}_{n} \backslash\left(u_{n}\right)$, $\left(u_{n+1}\right) \propto \vec{s}_{n+1},\left(u_{0}, u_{1}, \ldots, u_{n+1}\right) \in V R W^{<\omega}(\vec{w}), \vec{s}_{n+1} \prec \vec{s} \backslash u_{1} * \ldots * u_{n}$ and, according to condition (ii), [s $\left.\odot \mathbf{t}, \vec{s}_{n+1}\right] \in \mathcal{R}$ for every $\mathbf{t} \in V R W^{<\omega}\left(\left(u_{1}, \ldots, u_{n}\right)\right)$.

Set $\vec{u}=\left(s_{1}, \ldots, s_{k}, u_{1}, u_{2}, \ldots\right) \in \mathcal{W}^{\omega}(\Sigma ; v)$ in case $\mathbf{s}=\left(s_{1}, \ldots, s_{k}\right)$ and $\vec{u}=$ $\left(u_{1}, u_{2}, \ldots\right)$ in case $\mathbf{s}=\emptyset$. Then $\vec{u} \in[\mathbf{s}, \vec{s}]$, since $\left(u_{1}, u_{2}, \ldots, u_{n}\right) \in V R W^{<\omega}(\vec{s})$ for every $n \in \mathbb{N}$. Let $\vec{t} \prec \vec{u} \backslash \mathbf{s}$ and $\mathbf{t} \neq \emptyset$ with $\mathbf{t} \propto \vec{t}$. Since $\mathbf{t} \in V R W^{<\omega}(\vec{u} \backslash \mathbf{s})$ we can define $n_{0}=\min \left\{n \in \mathbb{N}: \mathbf{t} \in V R W^{<\omega}\left(\left(u_{1}, \ldots, u_{n}\right)\right)\right\}$. Then $\left[\mathbf{s} \odot \mathbf{t}, \vec{s}_{n_{0}+1}\right] \in \mathcal{R}$. According to assumption (ii), we have that $\left[\mathbf{s} \odot \mathbf{t}, \vec{u} \backslash\left(s_{1}, \ldots, s_{k}, u_{1}, \ldots, u_{n_{0}}\right)\right] \in \mathcal{R}$ and, since $\vec{t} \backslash \mathbf{t} \prec \vec{u} \backslash \mathbf{s} \odot\left(u_{1}, \ldots, u_{n_{0}}\right) \prec \vec{s}_{n_{0}+1}$, we have that $[\mathbf{s} \odot \mathbf{t}, \vec{t} \backslash \mathbf{t}] \in \mathcal{R}$. In case $\mathbf{t}=\emptyset$, we have that $[\mathbf{s}, \vec{u} \backslash \mathbf{s}] \in \mathcal{R}$, since $\left[\mathbf{s}, \vec{s}_{1}\right] \in \mathcal{R}$. Hence, according to assumption (ii), $[\mathbf{s}, \vec{t}] \in \mathcal{R}$.

Proof of Theorem 6.2. Set

$$
\begin{aligned}
\mathcal{R}_{\mathcal{U}}=\left\{[\mathbf{t}, \vec{t}]:(\mathbf{t}, \vec{t}) \in W^{<\omega}(\Sigma ; v) \times W^{\omega}(\Sigma ; v),\right. \text { and } \\
\text { either } \left.[\mathbf{t}, \vec{t}] \cap \mathcal{U}=\emptyset, \quad \text { or } \quad\left[\mathbf{t}, \vec{t}_{1}\right] \cap \mathcal{U} \neq \emptyset \text { for every } \overrightarrow{t_{1}} \prec \vec{t}\right\} .
\end{aligned}
$$

It is easy to check that $\mathcal{R}_{\mathcal{U}}$ satisfies assumptions (i) and (ii) of Lemma 6.3, hence, there exists $\vec{u}=\left(u_{n}\right)_{n \in \mathbb{N}} \in[\mathbf{s}, \vec{s}]$ such that $[\mathbf{s} \odot \mathbf{t}, \vec{t} \backslash \mathbf{t}] \in \mathcal{R}_{\mathcal{U}}$ for every $\vec{t} \prec \vec{u} \backslash \mathbf{s}$ and $\mathbf{t} \propto \vec{t}$.

For $\mathbf{t} \in V R W^{<\omega}(\vec{u} \backslash \mathbf{s})$ there exists unique $\vec{u}_{\mathbf{t}} \prec \vec{u} \backslash \mathbf{s}$ with $\mathbf{t} \propto \vec{u}_{\mathbf{t}}$ and $\vec{u}_{\mathbf{t}} \backslash \mathbf{t}=$ $\left(u_{n}\right)_{n>n_{0}}$ for some $n_{0} \in \mathbb{N}$. Then $\left[\mathbf{s} \odot \mathbf{t}, \vec{u}_{\mathbf{t}} \backslash \mathbf{t}\right] \in \mathcal{R}_{\mathcal{U}}$. Set $\mathcal{F}=\left\{\mathbf{t} \in V R W^{<\omega}(\vec{u} \backslash \mathbf{s})\right.$ : $\left[\mathbf{s} \odot \mathbf{t}, \vec{u}_{1}\right] \cap \mathcal{U} \neq \emptyset$ for every $\left.\vec{u}_{1} \prec \vec{u}_{\mathbf{t}} \backslash \mathbf{t}\right\}$. 
The family $\mathcal{F}$ is a tree. Indeed, let $\mathbf{t} \in \mathcal{F}$ and $\mathbf{t}_{1} \propto \mathbf{t}$. Since $\mathbf{t}_{1} \in V R W^{<\omega}(\vec{u} \backslash \mathbf{s})$, we have that $\left[\mathbf{s} \odot \mathbf{t}_{1}, \vec{u}_{\mathbf{t}_{1}} \backslash \mathbf{t}_{1}\right] \in \mathcal{R}_{\mathcal{U}}$. But it is impossible that $\left[\mathbf{s} \odot \mathbf{t}_{1}, \vec{u}_{\mathbf{t}_{1}} \backslash \mathbf{t}_{1}\right] \cap \mathcal{U}=\emptyset$, since $\left[\mathbf{s} \odot \mathbf{t}, \vec{u}_{1}\right] \cap \mathcal{U} \neq \emptyset$ for every $\vec{u}_{1} \prec \vec{u}_{\mathbf{t}} \backslash \mathbf{t}$. Hence, $\left[\mathbf{s} \odot \mathbf{t}_{1}, \vec{u}_{1}\right] \cap \mathcal{U} \neq \emptyset$ for every $\vec{u}_{1} \prec \vec{u}_{\mathbf{t}_{1}} \backslash \mathbf{t}_{1}$, and consequently $\mathbf{t}_{1} \in \mathcal{F}$.

We now apply Theorem 5.4 for $\mathcal{F}$ and $\vec{u} \backslash \mathbf{s}$. We have the following cases:

[Case 1] There exists $\vec{u}_{1} \prec \vec{u} \backslash \mathbf{s} \prec \vec{s}$ such that $V R W^{<\omega}\left(\vec{u}_{1}\right) \subseteq \mathcal{F}$. This gives that $\left[\mathbf{s} \odot \mathbf{t}, \vec{u}_{2}\right] \cap \mathcal{U} \neq \emptyset$ for every $\mathbf{t} \in V R W^{<\omega}\left(\vec{u}_{1}\right)$ and $\vec{u}_{2} \prec \vec{u}_{1} \backslash \mathbf{t}$, which implies that $\left[\mathbf{s}, \vec{u}_{1}\right] \subseteq \hat{\mathcal{U}}$.

[Case 2] There exists a countable ordinal $\xi_{0}=\zeta_{\vec{u} \backslash \mathbf{s}}^{\mathcal{F}}=\zeta_{(\mathbf{s}, \vec{s})}^{\mathcal{U}}$ such that for every $\xi>$ $\xi_{0}$ there exists $\vec{u}_{1} \prec \vec{u} \backslash \mathbf{s} \prec \vec{s}$ with $W^{\xi}(\Sigma ; v) \cap V R W^{<\omega}\left(\vec{u}_{1}\right) \subseteq W^{<\omega}(\Sigma ; v) \backslash \mathcal{F}$. Using the canonical representation of every infinite sequense of variable words with respect to the family $W^{\xi}(\Sigma ; v)$ (Proposition 4.3 ), we have that $\left[\mathbf{s} \odot \mathbf{t}, \vec{u}_{1}\right] \subseteq W^{\omega}(\Sigma ; v) \backslash \mathcal{U}$ for every $\mathbf{t} \in W^{\xi}(\Sigma ; v) \cap V R W^{<\omega}\left(\vec{u}_{1}\right)$. . Hence, $[\mathbf{s} \odot \mathbf{t}, \vec{t} \backslash \mathbf{t}] \subseteq W^{\omega}(\Sigma ; v) \backslash \mathcal{U}$ for every $\vec{t} \prec \vec{u}_{1}$ and $\mathbf{t} \in W^{\xi}(\Sigma ; v)$ with $\mathbf{t} \propto \vec{t}$.

Applying Theorem 6.2 to partitions $\mathcal{U}$ that are closed or meager in the topology $\mathfrak{T}_{E}$, we obtain the following consequences.

Corollary 6.4. Let $\mathcal{U}$ be a subset of $W^{\omega}(\Sigma ; v)$ closed in the topology $\mathfrak{T}_{E}, \mathbf{s} \in$ $W^{<\omega}(\Sigma ; v)$ and $\vec{s} \in W^{\omega}(\Sigma ; v)$. Then

either there exists $\vec{u} \prec \vec{s}$ such that $[\mathbf{s}, \vec{u}] \subseteq \mathcal{U}$,

or there exists a countable ordinal $\xi_{0}$ such that for every $\xi>\xi_{0}$ there exists $\vec{u} \prec \vec{s}$ with $[\mathbf{s} \odot \mathbf{t}, \vec{t} \backslash \mathbf{t}] \subseteq W^{\omega}(\Sigma ; v) \backslash \mathcal{U}$ for every $\vec{t} \prec \vec{u}$ and $\mathbf{t} \in W^{\xi}(\Sigma ; v)$ with $\mathbf{t} \propto \vec{t}$.

Corollary 6.5. Let $\mathcal{U}$ be a subset of $W^{\omega}(\Sigma ; v)$ meager in the topology $\mathfrak{T}_{E}, \mathbf{s} \in$ $W^{<\omega}(\Sigma ; v)$ and $\vec{s} \in W^{\omega}(\Sigma ; v)$. Then, there exists a countable ordinal $\xi_{0}$ such that for every $\xi>\xi_{0}$ there exists $\vec{u} \prec \vec{s}$ with $[\mathbf{s} \odot \mathbf{t}, \vec{t} \backslash \mathbf{t}] \subseteq W^{\omega}(\Sigma ; v) \backslash \mathcal{U}$ for every $\vec{t} \prec \vec{u}$ and $\mathbf{t} \in W^{\xi}(\Sigma ; v)$ with $\mathbf{t} \propto \vec{t}$.

Proof. We use Theorem 6.2 for $\mathcal{U}$. We will prove that the first alternative is impossible. Indeed, let $\vec{u} \prec \vec{s}$ such that $[\mathbf{s}, \vec{u}] \subseteq \hat{\mathcal{U}}$. If $\mathcal{U}=\bigcup_{n \in \mathbb{N}} \mathcal{U}_{n}$ with $\left(\hat{\mathcal{U}}_{n}\right)^{\diamond}=\emptyset$ for every $n \in \mathbb{N}$, then we set

$$
\begin{aligned}
\mathcal{R}=\{[\mathbf{t}, \vec{t}]: \mathbf{t} & \in W^{<\omega}(\Sigma ; v), \vec{t} \in W^{\omega}(\Sigma ; v) \text { and } \\
& {\left.[\mathbf{t}, \vec{t}] \cap \mathcal{U}_{k}=\emptyset \text { for every } k \in \mathbb{N} \text { with } k \leq|\mathbf{t}|\right\}, }
\end{aligned}
$$

where $|\mathbf{t}|$ denotes the number of terms of the finite sequence $\mathbf{t} \in W^{<\omega}(\Sigma ; v)$. The family $\mathcal{R}$ obviously satisfies assumption (ii) of Lemma 6.3 and also satisfies assumption (i) of Lemma 6.3. Indeed, according to Theorem 6.2 and Proposition 4.3, for every $\mathbf{t} \in W^{<\omega}(\Sigma ; v), \vec{t} \in W^{\omega}(\Sigma ; v)$ and $k \in \mathbb{N}$ there exists $\vec{t}_{1} \prec \vec{t}$ such that $\left[\mathbf{t}, \vec{t}_{1}\right] \cap \mathcal{U}_{k}=\emptyset$, as it is impossible that $\left[\mathbf{t}, \vec{t}_{1}\right] \subseteq \hat{\mathcal{U}}_{k}$ for some $k \in \mathbb{N}$. Hence, according to Lemma 6.3 , there exists $\vec{u}_{1} \in[\mathbf{s}, \vec{u}]$ such that $\left[\mathbf{s} \odot \mathbf{t}, \vec{u}_{1} \backslash \mathbf{s} \odot \mathbf{t}\right] \in \mathcal{R}$ for every $\mathbf{t} \in V R W^{<\omega}\left(\vec{u}_{1} \backslash \mathbf{s}\right)$.

We will prove that $\left[\mathbf{s}, \vec{u}_{1} \backslash \mathbf{s}\right] \cap \mathcal{U}=\emptyset$. Let $\vec{u}_{2} \in\left[\mathbf{s}, \vec{u}_{1} \backslash \mathbf{s}\right] \cap \mathcal{U}_{k}$ for some $k \in \mathbb{N}$. Then there exists $\mathbf{t} \in V R W^{<\omega}\left(\vec{u}_{1} \backslash \mathbf{s}\right)$ such that $\mathbf{s} \odot \mathbf{t} \propto \vec{u}_{2}, k \leq|\mathbf{s} \odot \mathbf{t}|$ and $\left[\mathbf{s} \odot \mathbf{t}, \vec{u}_{1} \backslash \mathbf{s} \odot \mathbf{t}\right] \cap \mathcal{U}_{k} \neq \emptyset$. So, $\left[\mathbf{s} \odot \mathbf{t}, \vec{u}_{1} \backslash \mathbf{s} \odot \mathbf{t}\right] \notin \mathcal{R} ;$ a contradiction, since $\mathbf{t} \in V R W^{<\omega}\left(\vec{u}_{1} \backslash \mathbf{s}\right)$. Hence, $\left[\mathbf{s}, \vec{u}_{1} \backslash \mathbf{s}\right] \cap \mathcal{U}=\emptyset$, and consequently $\vec{u}_{1} \notin \hat{\mathcal{U}}$.

This is a contradiction, since $\vec{u}_{1} \in[\mathbf{s}, \vec{u}] \subseteq \hat{\mathcal{U}}$. Hence, the first alternative of Theorem 6.2 for the partition $\mathcal{U}$ is impossible, so the second alternative holds for $\mathcal{U}$. 
We recall the definition of the completely Ramsey families of infinite sequences of variable words given by Carlson in [C].

Definition 6.6. A family $\mathcal{U} \subseteq W^{\omega}(\Sigma ; v)$ of infinite sequences of variable words on a finite, non-empty alphabet $\Sigma$ is called completely Ramsey if for every $\mathbf{s} \in W^{<\omega}(\Sigma ; v)$ and every $\vec{s} \in W^{\omega}(\Sigma ; v)$ there exists $\vec{u} \prec \vec{s}$ such that

$$
\text { either }[\mathbf{s}, \vec{u}] \subseteq \mathcal{U}, \quad \text { or } \quad[\mathbf{s}, \vec{u}] \subseteq W^{\omega}(\Sigma ; v) \backslash \mathcal{U} .
$$

The characterization of completely Ramsey families of infinite sequences of variable words, proved with a different method by Carlson in $[\mathrm{C}]$, is a consequence of Theorem 6.2.

Corollary 6.7 (Carlson, $[\mathrm{C}]) . A$ family $\mathcal{U} \subseteq W^{\omega}(\Sigma ; v)$ is completely Ramsey if and only if $\mathcal{U}$ has the Baire property in the topology $\mathfrak{T}_{E}$.

Proof. Let $\mathcal{U} \subseteq W^{\omega}(\Sigma ; v)$ have the Baire property in the topology $\mathfrak{T}_{E}$. Then $\mathcal{U}=\mathcal{B} \triangle \mathcal{C}=\left(\mathcal{B} \cup \mathcal{C}^{c}\right) \cup\left(\mathcal{C} \cap \mathcal{B}^{c}\right)$, where $\mathcal{B} \subseteq W^{\omega}(\Sigma ; v)$ is $\mathfrak{T}_{E}$-closed and $\mathcal{C} \subseteq W^{\omega}(\Sigma ; v)$ is $\mathfrak{T}_{E}$-meager $\left(\mathcal{C}^{c}=W^{\omega}(\Sigma ; v) \backslash \mathcal{C}\right)$. According to Corollary 6.5 and Proposition 4.3, for every $\mathbf{s} \in W^{<\omega}(\Sigma ; v)$ and $\vec{s} \in W^{\omega}(\Sigma ; v)$, there exists $\vec{u} \prec \vec{s}$ such that $[\mathbf{s}, \vec{u}] \subseteq \mathcal{C}^{c}$, and according to Corollary 6.4 , there exists $\vec{u}_{1} \prec \vec{u}$ such that

$$
\begin{aligned}
& \text { either }\left[\mathbf{s}, \vec{u}_{1}\right] \subseteq \mathcal{B} \cap[\mathbf{s}, \vec{u}] \subseteq \mathcal{B} \cap \mathcal{C}^{c} \subseteq \mathcal{U}, \\
& \text { or }\left[\mathbf{s}, \vec{u}_{1}\right] \subseteq \mathcal{B}^{c} \cap[\mathbf{s}, \vec{s}] \subseteq \mathcal{B}^{c} \cap \mathcal{C}^{c} \subseteq \mathcal{U}^{c} .
\end{aligned}
$$

Hence, $\mathcal{U}$ is completely Ramsey.

On the other hand, if $\mathcal{U}$ is completely Ramsey, then $\mathcal{U}=\mathcal{U}^{\diamond} \cup\left(\mathcal{U} \backslash \mathcal{U}^{\diamond}\right)$ and $\mathcal{U} \backslash \mathcal{U}^{\diamond}$ is a meager set in $\mathfrak{T}_{E}$. Hence $\mathcal{U}$ has the Baire property in the topology $\mathfrak{T}_{E}$.

A similar characterization of the completely Ramsey families of $W^{\omega}(\Sigma)$ can be proved analogously, as a consequence of Theorem 5.2.

Remark 6.8. (i) The Ellentuck topology $\mathfrak{T}_{E}$ on $W^{\omega}(\Sigma)$ has basic open sets of the form $[\mathbf{s}, \vec{s}]$ for $\mathbf{s} \in W^{<\omega}(\Sigma)$ and $\vec{s} \in W^{\omega}(\Sigma ; v)$, where

$$
[\mathbf{s}, \vec{s}]=\left\{\vec{w} \in W^{\omega}(\Sigma): \mathbf{s} \propto \vec{w} \quad \text { and } \quad \vec{w} \backslash \mathbf{s} \in R W^{\omega}(\vec{s})\right\} \text {, and }[\emptyset, \vec{s}]=R W^{\omega}(\vec{s}) \text {. }
$$

(ii) A family $\mathcal{U} \subseteq W^{\omega}(\Sigma)$ of infinite sequences of words on a finite, non-empty alphabet $\Sigma$ is called completely Ramsey if for every $\mathbf{s} \in W^{<\omega}(\Sigma)$ and every $\vec{s} \in$ $W^{\omega}(\Sigma ; v)$ there exists $\vec{u} \prec \vec{s}$ such that

$$
\text { either }[\mathbf{s}, \vec{u}] \subseteq \mathcal{U}, \quad \text { or } \quad[\mathbf{s}, \vec{u}] \subseteq W^{\omega}(\Sigma) \backslash \mathcal{U} .
$$

(iii) A family $\mathcal{U} \subseteq W^{\omega}(\Sigma)$ is completely Ramsey if and only if $\mathcal{U}$ has the Baire property in the topology $\mathfrak{T}_{E}$.

\section{ACKNOWLEDGMENTS}

We wish to thank the anonymous referee for constructive suggestions. We also wish to thank Cyprus University for the warm hospitality extended during our visit in the fall semester of 2005. Both authors were partially supported by an Athens University research grant. 


\section{REFERENCES}

[AA] D. Alspach and S. Argyros, Complexity of weakly null sequences, Dissertations Math. 321 (1992), 1-44. MR1191024 (93j:46014)

[AO] D. Alspach and E. Odell, Averaging weakly null sequences, Lecture Notes in Math. 1332, Springer, Berlin, 1988, pp. 126-144. MR967092 (89j:46014)

[AGR] S. Argyros, G. Godefroy and H. Rosenthal, Descriptive set theory and Banach spaces, Handbook of the geometry of Banach spaces 2, North-Holland, Amsterdam (2003), 10071069. MR1999190 (2004g:46002)

[B] J. Baumgartner, A short proof of Hindman's theorem, J. Combinatorial Theory, Ser. A, 17 (1974), 384-386. MR0354394 (50:6873)

[BBH] V. Bergelson, A. Blass, N. Hindman, Partition theorems for spaces of variable words, Proc. London Math.Soc. 68(1994),449-476. MR1262304 (95i:05107)

[C] T. Carlson, Some unifying principles in Ramsey theory, Discrete Math. 68 (1988), 117169. MR926120 (89b:04006)

[E] E. Ellentuck, A new proof that analytic sets are Ramsey, J. Symb. Logic 39 (1974), 163164. MR0349393 (50:1887)

[F1] V. Farmaki, Classifications of Baire-1 functions and co-spreading models, Trans. Amer. Math. Soc. 345 (2), (1994), 819-831. MR1262339 (96c:46017)

[F2] V. Farmaki, Ramsey dichotomies with ordinal index, arXiv: math. LO/9804063 v1, (1998), electronic prepublication.

[F3] V. Farmaki, Ramsey and Nash-Williams combinatorics via Schreier families, arXiv: math. FA/0404014 v.1, (2004), electronic prepublication.

[F4] V. Farmaki, The uniform convergence ordinal index and the $l^{1}-$ behavior of a sequence of functions, Positivity 8 (1), (2004), 49-74. MR2053575 (2005e:46029)

[FN] V. Farmaki, S. Negrepontis, Block Combinatorics, Trans. Amer. Math. Soc. 358 (2006), 2759-2779. MR2204055 (2006m:03073)

[FK] H. Furstenberg, Y. Katznelson Idempotents in compact semigroups and Ramsey theory, Israel J. Math. 68 (1989), 257-270. MR1039473 (92d:05170)

[G] W. T. Gowers, An infinite Ramsey theorem and some Banach space dichotomies, Annals of Mathematics 156 (2002), 797-833. MR1954235 (2005a:46032)

[GRS] R. Graham, B. Rothschild and J. Spencer, Ramsey Theory, Wiley, New York, 1990. MR1044995 (90m:05003)

[HJ] A. W. Hales, R. I.Jewett, Regularity and Positional Games, Trans. Amer. Math. Soc. 106 (1963), 222-229. MR0143712 (26:1265)

$[\mathrm{H}] \quad$ N. Hindman, Finite sums from sequences within cells of a partition of $\mathbb{N}$, J. Combinatorial Theory, Ser. A 17 (1974), 1-11. MR0349574 (50:2067)

[KS] J. Ketonen and R. Solovay, Rapidly growing Ramsey functions, Ann. of Math. 113 (1981), 267-314. MR607894 (84c:03100)

[K] K. Kuratowski, Topology, Volume I, Academic Press (1966). MR0217751 (36:840)

[KM] K. Kuratowski and A. Mostowski, Set Theory, North-Holland, Amsterdam, 1968. MR0229526 (37:5100)

[L] A. Levy, Basic Set Theory, Springer-Verlag, 1979. MR533962 (80k:04001)

[McC] R. McCutcheon, Elemental Methods in Ergodic Ramsey Theory, Lecture Notes in Math. 1722 Springer (1999). MR1738544 (2001c:05141)

[M] K. Milliken, Ramsey's theorem with sums or unions, J. Combinatorial Theory, Ser. A 18 (1975), 276-290. MR0373906 (51:10106)

[NW] C. St. J. A. Nash-Williams, On well quasiordering transfinite sequences, Proc. Camb. Phil. Soc. 61 (1965), 33-39. MR0173640 (30:3850)

[O] E. Odell, On subspaces, asymptotic structure, and distortion of Banach spaces; connections with logic, Analysis and Logic, London Math. Soc. Lecture Note Ser. 262, Cambridge Univ. Press, (2002), 189-267. MR1967836 (2004b:46016)

$[\mathrm{PH}]$ J. Paris and L. Harrington, A mathematical incompleteness in Peano arithmetic, Handbook of Mathematical Logic, North-Holland, Amsterdam (1977), 1133-1142. MR0457132 (56:15351)

[R] F. P. Ramsey, On a problem of formal logic, Proc. London Math. Soc. 30 (2), (1929), $264-286$. 
[S] J. Schreier, Ein Gegenbeispiel zur Theorie der schwachen Konvergenz, Studia Math. 2 (1930), 58-62.

[T1] A. Taylor, Some results in partition theory, Ph.D. dissertation, Dartmouth College, 1975.

[T2] A. Taylor, A canonical partition relation for finite subsets of $\omega$, J. Combinatorial Theory, Ser. A 21 (1976), 137-146. MR0424571 (54:12530)

[TJ] N. Tomczak-Jaegermann, Banach spaces of type p have arbitrarily distortable subspaces, Geom. As. Funct. Anal. 6 (1996), 1074-1082. MR1421875 (98g:46020)

[vdW] B. L. van der Waerden, Beweis einer Baudetschen Vermutung, Nieuw Arch. Wisk. 15 (1927), 212-216.

Department of Mathematics, Athens University, Panepistemiopolis, Athens 157 84, Greece

E-mail address: vfarmaki@math.uoa.gr

Department of Mathematics, Athens University, Panepistemiopolis, Athens 157 84, Greece

E-mail address: snegrep@math.uoa.gr 